\title{
Receiver Design for Signals in Non-Gaussian Noise: Applications to Symmetric Alpha-Stable and Middleton's Class-A Noise Models
}

\author{
by
}

Tarik Sayed Shehata Mohamed Saleh, B. Sc, M. Sc.

A thesis submitted to the Faculty of Graduate and Postdoctoral Affairs

in partial fulfillment of the requirements for the degree of

Doctor of Philosophy in Electrical and Computer Engineering

Ottawa-Carleton Institute for Electrical and Computer Engineering (OCIECE)

Department of Systems and Computer Engineering

Carleton University

Ottawa, Ontario, Canada, K1S 5B6

June 2012

Copyright @2012- Tarik Saleh 
Library and Archives

Canada

Published Heritage

Branch

395 Wellington Street

Ottawa ON K1A ON4

Canada
Bibliothèque et

Archives Canada

Direction du

Patrimoine de l'édition

395 , rue Wellington

Ottawa ON K1A ON4

Canada
Your file Votre référence

ISBN: 978-0-494-93688-7

Our file Notre référence

ISBN: 978-0-494-93688-7
NOTICE:

The author has granted a nonexclusive license allowing Library and Archives Canada to reproduce, publish, archive, preserve, conserve, communicate to the public by telecommunication or on the Internet, loan, distrbute and sell theses worldwide, for commercial or noncommercial purposes, in microform, paper, electronic and/or any other formats.

The author retains copyright ownership and moral rights in this thesis. Neither the thesis nor substantial extracts from it may be printed or otherwise reproduced without the author's permission.
AVIS:

L'auteur a accordé une licence non exclusive permettant à la Bibliothèque et Archives Canada de reproduire, publier, archiver, sauvegarder, conserver, transmettre au public par télécommunication ou par l'Internet, prêter, distribuer et vendre des thèses partout dans le monde, à des fins commerciales ou autres, sur support microforme, papier, électronique et/ou autres formats.

L'auteur conserve la propriété du droit d'auteur et des droits moraux qui protege cette thèse. $\mathrm{Ni}$ la thèse ni des extraits substantiels de celle-ci ne doivent être imprimés ou autrement reproduits sans son autorisation.
In compliance with the Canadian Privacy Act some supporting forms may have been removed from this thesis.

While these forms may be included in the document page count, their removal does not represent any loss of content from the thesis.
Conformément à la loi canadienne sur la protection de la vie privée, quelques formulaires secondaires ont été enlevés de cette thèse.

Bien que ces formulaires aient inclus dans la pagination, il n'y aura aucun contenu manquant. 


\section{Abstract}

Non-Gaussian impulsive noise has been used to model different noise sources in many communication systems, such as multiple access interference, man-made electromagnetic noise, car ignition and mechanical switching and many others. There have been different statistical distributions proposed to model such impulsive noise such as the Gaussian mixture distribution, Middleton's Class A noise model, and the symmetric alpha stable distribution. However, the optimal receivers designed for channels based on these distributions are very complex. In this thesis, we discuss the problem of designing robust low complexity receivers with near-optimal performance for signals in symmetric alpha stable noise. The research results are also applied to Class A noise.

In the detection part, we analyze the behavior of the optimal detector by using the optimal decision regions framework. Based on this framework, we have proposed a novel method to significantly improve the performance of simple suboptimal detectors; however, there are some limitations when the dimension of the decision regions becomes higher than 2. Another framework which is based on the optimal log-likelihood ratio is proposed to give a generalized approach to design simple suboptimal detectors

with near-optimal performance by using a piecewise linear approximation of the log likelihood ratio. The proposed framework has been also applied to design effective yet simple signal combiner for signals in multipath fading channel.

In the decoding part, we use the optimal LLR as a unified metric to propose 
a simple approach to justify and design low complexity suboptimal decoders. The proposed approach has been used to design suboptimal Viterbi and MAP decoders which give near optimal performance with low complexity. The proposed approach can also be used with other types of codes such as turbo and LDPC codes.

Moreover, the proposed LLR-based framework can be used to design simple receivers with different complex non-Gaussian models. In this thesis, we show that the LLR-based framework can be used with the case of the Middleton's Class A noise model to design a low-complexity detector and a simple Viterbi decoder as examples of the generality of the proposed framework. 
To my family, 


\section{Acknowledgments}

I am so grateful to God (Allah) for his uncountable bounties on me including ability to perform this research and to write this thesis. I would like also to acknowledge my thesis supervisors, Prof. Ian Marsland and Prof. Mohamed El-Tanany, for their patience, guidance, encouragement, support and help during the course of this research. Finally, I would like to express my deep gratitude to all my family members and friends for their support and prayers. 


\section{Table of Contents}

Abstract $\quad$ iii

Acknowledgments $\quad$ vi

Table of Contents vii

List of Tables $\quad$ xii

List of Figures $\quad$ xiii

List of Acronyms xviii

List of Symbols $\quad \mathbf{x x}$

1 Introduction 1

1.1 Motivation . . . . . . . . . . . . . . . . . 1

1.2 Non-Gaussian Impulsive Models . . . . . . . . . . . . . . . . . 2

1.2.1 The Gaussian Mixture Model . . . . . . . . . . . . . 2

1.2.2 The Middleton Class A Model . . . . . . . . . . . . . . 3

1.2.3 The Symmetric Alpha-Stable Model . . . . . . . . . . . 3

1.3 Research Objectives . . . . . . . . . . . . . . . 6

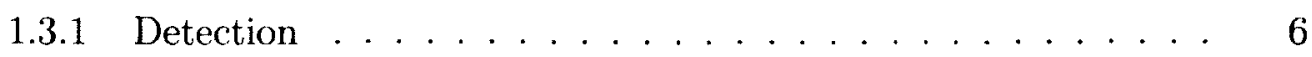

1.3.2 Decoding ................... 7 
1.3.3 Application to the Class A Noise Model . . . . . . . . . . 7

1.4 Research Contributions of the Thesis . . . . . . . . . . . . 8

1.5 Thesis Organization . . . . . . . . . . . . . . . 10

2 Symmetric Alpha-Stable: A Model for Impulsive Noise 11

2.1 Properties of the Alpha-Stable Distribution . . . . . . . . . . . . . . 11

2.2 Definition of Signal-to-Noise Ratio in The Context of S $\alpha \mathrm{S}$ Noise . . 15

2.3 System Model . . . . . . . . . . . . . . . . . . . . . 17

3 Signal Detection in SaS Noise: Background and Literature Review 18

3.1 Introduction . . . . . . . . . . . . . . . . 18

3.2 Optimal Maximum Likelihood Detector . . . . . . . . . . . . . . . . 19

3.3 Suboptimal Detectors . . . . . . . . . . . . . . . 20

3.3 .1 Cauchy Detector .................... 20

3.3.2 Myriad Filter . . . . . . . . . . . . . . 21

3.3.3 Gaussian Detector ................. 21

3.3.4 Soft Limiter (SL) Detector . . . . . . . . . . . . . . . 22

3.3.5 Locally Optimal Bayesian (LOB) Detector . . . . . . . . . . 23

3.3.6 Sign Correlator (SC) Detector . . . . . . . . . . . . . . 24

3.4 Performance Comparison and Discussion . . . . . . . . . . . . 25

3.4.1 Verification of Simulation ............. 25

3.4 .2 Simulation Setup . . . . . . . . . . . . . . . . 26

3.4.3 Performance of the Optimal Detector . . . . . . . . . . 27

3.4.4 Performance of the Suboptimal Detectors . . . . . . . . . . 32

3.4.5 Summary ...................... 35

4 Optimal Decision Regions: A Framework to Design Suboptimal $\begin{array}{ll}\text { Detectors for Signals in SaS Noise } & 36\end{array}$ 
4.1 Introduction . . . . . . . . . . . . . . . . 36

4.2 Optimal Decision Regions . . . . . . . . . . . . . 37

4.3 Performance Justification of the Existing Detectors . . . . . . . . . . 41

4.3 .1 Gaussian Detector . . . . . . . . . . . . . . 42

4.3 .2 Soft Limiter Detector . . . . . . . . . . . . . . . . . . 42

4.3 .3 Cauchy Detector . . . . . . . . . . . . . . 42

4.3 .4 Myriad Filter . . . . . . . . . . . . . 43

4.3.5 Locally Optimum Baysian Detector . . . . . . . . . . . . . 43

4.3.6 Sign Correlator Detector . . . . . . . . . . . . . . 44

4.4 Proposed Simplified Near-Optimal Detectors . . . . . . . . . . . . . . 44

4.4.1 Soft Limiter Detector with an Adaptive Threshold . . . . . . . 45

4.4.1.1 Soft Limiter Detector Analysis . . . . . . . . . . 45

4.4.1.2 Adaptive Soft Limiter (ASL) Detector . . . . . . . 47

4.4.1.3 Simulation Results and discussion . . . . . . . . 47

4.4.2 Low-Complexity Piecewise Detector . . . . . . . . . . . 51

4.4.2.1 Performance Evaluation and Simulation Results . . . 53

4.5 Conclusion . . . . . . . . . . . . . . . . 60

5 Log-likelihood Ratios: A Generalized Framework to Design Suboptimal Detectors for Signals in SaS Noise $\quad 61$

5.1 Introduction . . . . . . . . . . . . . . . 61

5.2 Piecewise Linear LLR Approximation . . . . . . . . . . . . . . . . . 63

5.2.1 A Simplified Approximation of the PWL-LLR Parameters . . 73

5.2.2 Decision Boundaries of PWL-LLR Detector . . . . . . . . . . 75

5.2.3 Performance Evaluation of the PWL-LLR Detector . . . . . 76

5.3 The PWL-LLR Diversity Combiner for Fading Channels . . . . . . . 80

5.3.1 Optimal and Suboptimal Detectors for Fading Channels . . . 81 
5.3.2 The PWL-LLR Diversity Combiner . . . . . . . . . . . . 82

5.3.3 Performance Comparison . . . . . . . . . . . . 83

5.4 Conclusion . . . . . . . . . . . . . . . . . . . 87

\section{Log-likelihood Ratios: A Method to Design Suboptimal Decoders} $\begin{array}{ll}\text { for Signals in SaS Noise } & 89\end{array}$

6.1 Introduction . . . . . . . . . . . . . . . 89

6.2 Literature Review . . . . . . . . . . . . . . . . . . . . . . . . . . . 89

6.2 .1 Viterbi Decoding in $\mathrm{S} \boldsymbol{\alpha} \mathrm{S}$ Noise . . . . . . . . . . . 90

6.2.1.1 Optimal and Suboptimal Viterbi Decoders . . . . . 90

6.2.1.1.1 ML Viterbi Decoder . . . . . . . . . . 91

6.2.1.1.2 Gaussian Viterbi Decoder ... . . . . . 91

6.2.1.1.3 Absolute-Branch Metric Viterbi Decoder . . 92

6.2.1.2 Performance Evaluation . . . . . . . . . . . 92

6.2 .2 MAP Decoding in Sas Noise . . . . . . . . . . . 94

6.2.2.1 Optimal and Suboptimal MAP Decoder . . . . . 95

$6.2 .2 .2 \quad$ Performance Evaluation $\ldots \ldots \ldots$

6.2 .3 Summary . . . . . . . . . . . . . . . . 98

6.3 Design Near-Optimal Decoders using the LLR-Based Framework . . . 100

6.3.1 Proposed Suboptimal Viterbi Decoder . . . . . . . . . 101

6.3.1.1 Performance Justification of the Optimal and Suboptimal Viterbi Algorithms using the LLR-Framework . 101

6.3.1.2 The Dual Absolute Branch Metric: Implicit Use of LLR104 6.3.1.2.1 Performance Evaluation and Simulation Results ..................... 106

6.3.1.3 The PWL-LLR Branch Metric . . . . . . . . . . . . 109 
6.3.1.3.1 Performance Evaluation and Simulation Results ................. 110

6.3.2 Proposed Suboptimal MAP Decoder . . . . . . . . . . 113

6.3.2.1 Log-MAP Decoder Based on PWL-LLR . . . . . . 113

6.3.2.2 Performance Evaluation . . . . . . . . . . 115

6.4 Conclusion . . . . . . . . . . . . . . . 118

7 LLR-Based Framework: Applications to Detector and Decoder Design for Signals in Middleton's Class A noise $\quad 119$

7.1 Introduction . . . . . . . . . . . . . . 119

7.2 Detector Design . . . . . . . . . . . . . . . . . 120

7.2 .1 System Model . . . . . . . . . . . . . . . 120

7.2 .2 Optimal and Suboptimal Detectors . . . . . . . . . . 123

7.2 .3 PWL-LLR Design for Class A Noise . . . . . . . . . . . . 124

7.2.4 Performance Evaluation and Simulation Results . . . . . . 127

7.3 Decoder Design . . . . . . . . . . . . . . . . . 132

7.3.1 Optimal and Suboptimal Viterbi Decoding . . . . . . . . . 132

7.3.2 Performance of the PWL-LLR Viterbi Decoder . . . . . . 133

7.3 .3 Conclusion . . . . . . . . . . . . . . . . 134

8 Conclusion and Future Work $\quad 136$

8.1 Conclusion . . . . . . . . . . . . . . . . 136

8.2 Future Work . . . . . . . . . . . . . . . . 139

8.2.1 Channel Estimation in $\mathrm{S} \boldsymbol{\alpha} \mathrm{S} \ldots \ldots . . \ldots 139$

8.2 .2 LLR Estimation . . . . . . . . . . . . . . . 140

$\begin{array}{ll}\text { List of References } & 141\end{array}$ 


\section{List of Tables}

6.1 Normalized MSE between the suboptimal and optimal LLR with $\mathrm{GSNR}=5 \mathrm{~dB} . \ldots \ldots \ldots \ldots \ldots$ 


\section{List of Figures}

2.1 Symmetric $\alpha$-stable distribution with $\gamma=1 \ldots \ldots \ldots$

2.2 Symmetric $\alpha$-stable distribution with $\gamma=1$ on $\log$ scale. . . . . 13

2.3 Realizations of symmetric $\alpha$-stable processes for different values of $\alpha$, with $\gamma=1 \ldots \ldots \ldots \ldots \ldots \ldots$

3.1 Block diagram of the soft limiter detector . . . . . . . . . . . . . 22

3.2 Block diagram of the locally optimal detector . . . . . . . . . . . 23

3.3 Locally optimal nonlinearity with $\alpha=1, \gamma=1 \ldots \ldots 24$

3.4 BER for the optimal detector for different $\gamma$ (with $N=10$ ). Reproduced results from $[50]$, Figure 5 -a. . . . . . . . . . . . 26

3.5 BER for the optimal detector for different $N$ (with $\alpha=1$ ) . . . . . 27

3.6 BER for the optimal detector for different $N$ (with $\alpha=1.3$ ). . . . . $\quad 28$

3.7 BER for the optimal detector for different $N$ (with $\alpha=1.5$ ). . . . . 28

3.8 BER for the optimal detector for different $N$ (with $\alpha=1.7$ ) . . . . 29

3.9 BER for the optimal detector for different $N$ (with $\alpha=1.9$ ). . . . . . 29

3.10 BER for the optimal detector for different $\alpha$ (with $N=1$ ) . . . . 30

3.11 BER for the optimal detector for different $\alpha$ (with $N=2$ ) . . . . . 30

3.12 BER for the optimal detector for different $\alpha$ (with $N=5$ ). . . . . 31

3.13 BER for the optimal detector for different $\alpha$ (with $N=10$ ). . . . . . 31

3.14 Performance comparison of suboptimal detectors with $\alpha=1.5, N=4$. 32

3.15 Performance comparison of suboptimal detectors with $\alpha=1.9, N=4 . \quad 33$ 
3.16 Performance of SL detector with different threshold values with $\alpha=$ $1.5, N=4 \ldots \ldots \ldots \ldots \ldots \ldots \ldots \ldots \ldots \ldots$

4.1 Optimal decision regions with $\alpha=1$ and $\gamma=1$. Shaded area: decide $s_{1}$, white area: decide $s_{0} \ldots \ldots \ldots \ldots \ldots \ldots \ldots \ldots$

4.2 Gaussian joint conditional probability density functions. . . . . . . 39

4.3 Cauchy joint conditional probability density functions. . . . . . . 39

4.4 Gaussian joint conditional probability density functions on log scale. . 40

4.5 Cauchy joint conditional probability density functions on log scale. . . 40

4.6 Optimal curved decision boundaries. . . . . . . . . . . . . 41

4.7 Comparison between the decision regions of the soft limiter (L), Gaus$\operatorname{sian}$ detectors $(G) \ldots \ldots \ldots \ldots \ldots \ldots \ldots$

4.8 Performance comparison of the ASL detector with $\alpha=1.5, N=2 \ldots \quad 49$

4.9 Performance comparison of the ASL detector with $\alpha=1.5, N=10$. . 49

4.10 Performance comparison of the ASL detector with $\alpha=1.9, N=10 . \quad . \quad 50$

4.11 Decision boundaries of the piecewise detector. . . . . . . . . 52

4.12 The excess probability of error $(\beta)$ evaluated at GSNR $=0,1, \ldots, 40 \mathrm{~dB}$ with $\alpha=1 \ldots \ldots \ldots \ldots \ldots \ldots \ldots \ldots \ldots \ldots \ldots \ldots \ldots \ldots \ldots \ldots$

4.13 Analytical and simulated probability of error with $\alpha=1$. Analytic: solid lines, simulations: markers only. . . . . . . . . . . . . 57

4.14 Performance comparison with $\alpha=0.7 \ldots \ldots \ldots \ldots \ldots$

4.15 Performance comparison with $\alpha=1.5 \ldots \ldots \ldots \ldots \ldots$

4.16 Performance comparison with $\alpha=1.9 \ldots \ldots \ldots \ldots \ldots$

4.17 Optimal decision regions for $N=3$ and $\alpha=1 \ldots \ldots \ldots \ldots$

4.18 A cross section in the optimal decision regions for $N=3$ and $\alpha=1 . \quad 60$

5.1 Optimal LLR with GSNR $=0 \mathrm{~dB} . \ldots \ldots \ldots \ldots$

5.2 Optimal LLR with GSNR $=5 \mathrm{~dB} \ldots \ldots \ldots \ldots \ldots$

5.3 Optimal LLR with GSNR $=10 \mathrm{~dB} \ldots \ldots \ldots \ldots \ldots$ 
5.4 Optimal LLR with GSNR $=15 \mathrm{~dB} \ldots \ldots . \ldots 65$

5.5 Optimal LLR for different values of GSNR with $\alpha=1$. . . . . . . . 66

5.6 Optimal LLR for different values of GSNR with $\alpha=1.3$. . . . . . . 66

5.7 Optimal LLR for different values of GSNR with $\alpha=1.5$. . . . . . . 67

5.8 Optimal LLR for different values of GSNR with $\alpha=1.7 \ldots \ldots 7$

5.9 Optimal LLR for different values of GSNR with $\alpha=1.9 . \ldots . . . .68$

5.10 The piecewise linear LLR (PWL-LLR) . . . . . . . . . . . . . 69

5.11 Exact and approximate values of $a . \ldots \ldots 71$

5.12 Exact and approximate values of $c \ldots \ldots \ldots 71$

5.13 Optimal values of $b$ for different values of $\alpha \ldots \ldots . \ldots 72$

5.14 Optimal values of $d$ for different values of $\alpha \ldots \ldots . \ldots 72$

5.15 Optimal values of $b$ for different values of GSNR. . . . . . . . . . . 73

5.16 Optimal values of $d$ for different values of GSNR. . . . . . . . . . . 73

5.17 Decision boundaries of the PWL-LLR detector compared to the optimal ones with $\alpha=1, N=2$. Solid: Optimal, Dashed: PWL_LLR. . . 75

5.18 Decision regions of the PWL-LLR with $\alpha=1$ and $N=3 \ldots \ldots 76$

5.19 Performance comparison of the PWL-LLR detector with $\alpha=1, N=2.77$

5.20 Performance comparison of the PWL-LLR detector with $\alpha=1, N=10.78$

5.21 Performance comparison of the PWL-LLR detector with $\alpha=1.5, N=4.79$

5.22 Performance comparison of the PWL-LLR detector with $\alpha=1.9, N=4.79$

5.23 Performance comparison of the PWL-LLR combiner with $\alpha=1.1$, $N=4 \ldots \ldots \ldots \ldots \ldots \ldots \ldots$

5.24 Performance comparison of the PWL-LLR combiner with $\alpha=1.8$, $N=4$.

5.25 Performance comparison of the PWL-LLR combiner with $\alpha=1.8$,

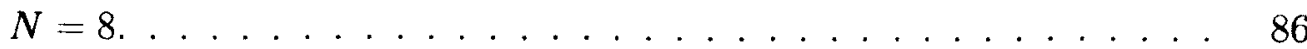

6.1 BER of the Viterbi decoder with different BMs with $\alpha=1 \ldots . . . .93$ 
6.2 BER of the Viterbi decoder with different BMs with $\alpha=1.7$. . . . . 94

6.3 BER of the MAP decoder with different BMs with $\alpha=1 \ldots \ldots 7$

6.4 BER of the MAP decoder with different BMs with $\alpha=1.7 \ldots \ldots$. . . 98

6.5 Optimal and suboptimal LLRs with $\alpha=1.3$ and GSNR $=5 \mathrm{~dB}$. . . 103

6.6 The ratio of the optimal scale factors $B_{2} / B_{1} \ldots \ldots \ldots$. . . . . . 106

6.7 BER of the Viterbi decoder with DAbs BM and $\alpha=1 \ldots \ldots 7$

6.8 BER of the Viterbi decoder with DAbs BM and $\alpha=1.5 . \ldots \ldots$. . . 108

6.9 BER of the Viterbi decoder with DAbs BM and $\alpha=1.7 \ldots \ldots 109$

6.10 BER of the Viterbi decoder with PWL-LLR and $\alpha=1 \ldots$. . . . . 111

6.11 BER of the Viterbi decoder with PWL-LLR and $\alpha=1.5 \ldots \ldots 112$

6.12 BER of the Viterbi decoder with PWL-LLR and $\alpha=1.7 \ldots$. . . . . . 112

6.13 Comparison between the optimal and different suboptimal LLRs. . . 115

6.14 BER of the MAP decoder with PWL-LLR and $\alpha=1 \ldots \ldots . . . .116$

6.15 BER of the MAP decoder with PWL-LLR and $\alpha=1.5 \ldots$. . . . . . 117

6.16 BER of the MAP decoder with PWL-LLR and $\alpha=1.7 \ldots \ldots . . .117$

7.1 The pdf of the Class A noise on a $\log$ scale with $\Gamma=0.1 \ldots \ldots 121$

7.2 The pdf of the Class A noise on a $\log$ scale with $\Gamma=0.01 . \quad \ldots \ldots . \quad 122$

7.3 The pdf of the Class A noise on a $\log$ scale with $A=0.1$. . . . . . 122

7.4 The pdf of the Class A noise on a $\log$ scale with $A=0.01$. . . . . . 123

7.5 The optimal LLR with SNR $=0 \mathrm{~dB} . \ldots \ldots \ldots . . \ldots . . \ldots 127$

7.6 The piecewise linear (PWL) simplified LLR. . . . . . . . . . . . . . 128

7.7 The conditional pdf, $\log f_{A, \Gamma}\left(r \mid s_{i}\right)$, and other terms. . . . . . . 128

7.8 Performance comparison with $A=0.1, \Gamma=0.1$ and $N=2 . \quad \ldots . \quad 129$

7.9 Performance comparison with $A=0.1, \Gamma=0.1$ and $N=5 . \quad \ldots \quad 130$

7.10 Performance comparison with $A=0.1, \Gamma=0.01$ and $N=2$. . . . 131

7.11 Performance comparison with $A=0.1, \Gamma=0.01$ and $N=5 . \ldots 131$ 
7.12 Performance of the proposed PWL LLR Viterbi decoder with $A=0.1$

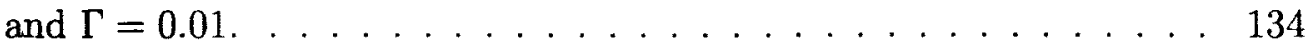




\section{List of Acronyms}

\begin{tabular}{ll}
\hline Acronyms & Definition \\
\hline APP & A posteriori probability. \\
ASL & Adaptive soft limiter. \\
Abs & Absolute value. \\
BER & Bit error rate. \\
BICM & Bit interleaved coded modulation. \\
BM & Branch metric. \\
BPSK & Binary phase shift key. \\
DAbs & Dual absolute value. \\
DFT & Discrete Fourier transform. \\
DS-SS & Direct sequence spread spectrum. \\
EMI & Electromagnetic interference. \\
FM & Frame error rate. \\
&
\end{tabular}

xviii 
GSNR Geometric signal-to-noise ratio.

LAPP Log a posteriori probability.

LDPC Low density parity check.

LLR Log likelihood ratio.

LOB Locally optimal Baysian.

MAP Maximum a posteriori.

ML $\quad$ Maximum likelihood.

PWL-LLR Piecewise linear LLR.

Pe Probability of error.

RV Random variable.

$\mathrm{S} \alpha \mathrm{S} \quad$ Symmetric alpha stable.

SC Sign correlator.

SL Soft limiter.

SNDR Signal power to noise dispersion ratio.

UWB Ultrawide band.

pdf probability density function. 


\section{List of Symbols}

\section{Symbols Definition}

\begin{tabular}{|c|c|}
\hline$\alpha$ & Characteristic exponent of the $\alpha$ stable RV. \\
\hline$\beta$ & Symmetry parameter of $\alpha$ stable RV. \\
\hline$\gamma$ & Dispersion of $\alpha$ stable RV. \\
\hline$\delta$ & Location parameter of $\alpha$ stable RV. \\
\hline$\eta$ & A threshold of a clipping device. \\
\hline$\kappa$ & The linearity parameter of the myriad filter. \\
\hline$\lambda$ & The test statistics of the a received symbol. \\
\hline$\mu$ & The mean of the additive $\mathrm{S} \alpha \mathrm{S}$ or Gaussian noise. \\
\hline$\sigma^{2}$ & The variance of the additive Gaussian noise. \\
\hline$\phi_{X}(\omega)$ & The characteristic function. \\
\hline$A$ & The amplitude of the transmitted signal. \\
\hline$C_{e}$ & Euler constant. \\
\hline$E_{b}$ & The energy per transmitted bit. \\
\hline
\end{tabular}


$L\left(b_{i} \mid \underline{\mathbf{r}}\right) \quad$ The log of the APP at the output of the MAP decoder.

$N \quad$ The number of samples per symbol collected at the receiver.

$R_{c} \quad$ The code rate.

$n(t) \quad$ The noise random process at the receiver front-end.

$n_{i} \quad$ The noise samples at the output of the matched filter.

$n_{c} \quad$ The number of coded bits.

$r(t) \quad$ The received random process at the receiver front-end.

$r_{i} \quad$ The received samples at the output of the matched filter.

$s(t) \quad$ The transmitted waveform.

sgn The signum function. 


\section{Chapter 1}

\section{Introduction}

\subsection{Motivation}

The Gaussian distribution has long been used as the default model for the thermal noise at the receiver of communication systems. It is statistically supported by the central limit theorem and it is justified by the physical properties of the noise. Nevertheless, measurements of the ambient noise in many physical channels show a non-Gaussian behavior. Experimental measurements in outdoor and indoor mobile radio channels as well as digital subscriber loop (DSL) systems show the impulsive nature of the ambient noise due to man-made electromagnetic interference as well as natural noise [1-6]. Car ignition and electo-mechanical switching are also shown to produce impulsive interference in a wide frequency band [7]. Moreover, multiple access interference in some wireless and ultra wideband (UWB) networks is characterized by impulsive non-Gaussian models [8-11]. Impulsive noise also appears in acoustic underwater channels [12-14], power line channels [15] and radar/sea clutter $[16,17]$.

It has been shown that the non-Gaussian noise that appears in different communication channels is heavy-tail in nature and is best described by a heavy-tailed statistical distribution with an algebraic tail rather than the exponential tail distributions such as the Gaussian model $[4,18]$. There are many heavy-tailed distributions 
that have been used to model non-Gaussian noise such as the Gaussian mixture [19], the Middleton class A noise model [18] and the symmetric alpha-stable (S $\alpha$ S) distribution $[8]$.

\section{$1.2 \quad$ Non-Gaussian Impulsive Models}

\subsubsection{The Gaussian Mixture Model}

The Gaussian mixture (GM) densities have been used to model a variety of nonGaussian noise environments $[19,20]$. Its probability density function is given by the weighted sum of $N$ Gaussian probability density functions as

$$
f_{G M}(x)=\sum_{i=1}^{N} c_{i} f_{G}\left(x ; \mu_{i}, \sigma_{i}^{2}\right)
$$

where $c_{i}$ are the weights and $f_{G}\left(x ; \mu_{i}, \sigma_{i}^{2}\right)$ is the Gaussian probability density function (pdf) with mean $\mu_{i}$ and variance $\sigma_{i}^{2}$. This model is quite general and can be used to approximate different symmetric, zero-mean densities such as the Laplacian distribution [21] and the $\mathrm{S} \alpha \mathrm{S}$ distribution [22]. Also, the Middleton class A noise model directly follows a GM density function with an infinite number of components.

A popular GM model is the two component $\epsilon$-mixture model:

$$
f_{X}(x)=(1-\epsilon) f_{G}\left(x ; 0, k \sigma^{2}\right)+\epsilon f_{G}\left(x ; 0, \sigma^{2}\right)
$$

where $0<\epsilon<1,0<k<1$. The second component, with a larger variance, models the impulsive noise with a probability of occurrence equal to $\epsilon$. The $\epsilon$-mixture model has been used as a mathematically tractable model for impulsive noise in many communication channels [23-26]. 


\subsubsection{The Middleton Class A Model}

Middleton has developed three statistical-physical noise models, known as the class A, $\mathrm{B}$ and $\mathrm{C}$ models $[18,27]$. These noise classes provide a broad, canonical description of many non-Gaussian noise processes for electromagnetic and acoustic environments. The three classes, A, B and C, are characterized by the relative bandwidth of the noise and the receiver. The pdf of Class A noise is a mixture of an infinite number of Gaussian distributions,

$$
f_{A}(x)=\sum_{n=0}^{\infty} f_{G}\left(x ; 0, \sigma_{n}^{2} \sigma^{2}\right)
$$

where $\sigma_{n}^{2}=\left(n / A+\Gamma_{p}\right) /\left(1+\Gamma_{p}\right)$. The pdf is described by three parameters, $A \geq 0$,

$\sigma^{2}>0$ and $\Gamma_{p} \geq 0$ which describe the intensity with which impulsive events occur, the power of the noise and the ratio of the powers of the Gaussian and non-Gaussian components, respectively. Middleton's Class A model has been used to model the electromagnetic interference in different communication systems $[10,28]$.

\subsubsection{The Symmetric Alpha-Stable Model}

The symmetric alpha-stable $(\mathrm{S} \alpha \mathrm{S})$ distribution, with symmetric pdf, describes a broad class of impulsive random variables with heavy tailed distributions [8]. The S $\alpha \mathrm{S}$ distribution has been used to model many impulsive noise sources using different analytical and empirical methodologies. The distribution has no closed form expression except for special cases when $\alpha=1$ (Cauchy distribution) and $\alpha=2$ (Gaussian distribution), so it is best described using its characteristic function

$$
\phi_{X}(\omega)=e^{-\gamma|\omega|^{\alpha}}
$$


where $\alpha$ measures the thickness of the tail of the distribution and $\gamma$ measures the spread of the distribution around its center. The probability density function is

$$
f_{X}(x)=\frac{1}{2 \pi} \int_{-\infty}^{\infty} \phi_{X}(\omega) e^{j \omega x} d \omega
$$

The $\mathrm{S} \alpha \mathrm{S}$ distribution is strictly a theoretical model as it possesses unrealistic properties such as infinite variance. However, it has been shown that this model is able to capture, to a great extent, the impulsive behavior of many physical noise sources. Moreover, the $\mathrm{S} \alpha \mathrm{S}$ model has attractive theoretical motivations such as the stability property and the support of the generalized limit theorem. The stability property means that a linear combination of $\mathrm{S} \alpha \mathrm{S}$ random variables results in a $\mathrm{S} \alpha \mathrm{S}$ random variable with different distribution parameters.

For the aforementioned reasons, the $\mathrm{S} \alpha \mathrm{S}$ distribution has been widely used to model impulsive noise in many different applications, including communication systems. In [29], the $\mathrm{S} \alpha \mathrm{S}$ distribution has been used to model the multiple access interference in direct sequence spread spectrum (DS-SS) networks. In [30,31], it was shown that the aggregate interference, which results from wireless terminals distributed in a plane according to a Poisson point process, can be modeled with the $\mathrm{S} \alpha \mathrm{S}$ distribution. Also, in [9], a mathematical framework based on the $\mathrm{S} \alpha \mathrm{S}$ distribution has been used to characterize the interference in many wireless networks such as cognitive radio and UWB networks. Moreover, the $\mathrm{S} \alpha \mathrm{S}$ model has been used to model co-channel interference [28]. In [11], it was shown that the $\mathrm{S} \alpha \mathrm{S}$ distribution closely fits the distribution of the multiple access interference in UWB networks. Recently, in [32], the $\mathrm{S} \alpha \mathrm{S}$ distribution has been proposed to model the multiple access interference in ad hoc UWB networks. In [33], the $\mathrm{S} \alpha \mathrm{S}$ model has been used in modeling interference in cooperative transmission in UWB relays. In [34], the $\mathrm{S} \alpha \mathrm{S}$ random process has been used to model the UWB channel impulse response at $60 \mathrm{GHz}$. Also, the 
$\mathrm{S} \alpha \mathrm{S}$ model has shown to be a close fit for the measured electromagnetic interference (EMI) generated by the clocks and buses in laptop and desktop computer environments [10]. In [6] a study of the impact of the EMI from household appliances on digital subscriber loop systems shows that the amplitude probability density function of the interference measured over the ADSL1 spectrum is well modeled by the $\mathrm{S} \alpha \mathrm{S}$ distribution.

In radar applications, the $\mathrm{S} \alpha \mathrm{S}$ distribution has been used to model the forest clutter observed in the UWB radar imagery [16]. Also, in [17], it has been shown that signal detection algorithms, based on the $\mathrm{S} \alpha \mathrm{S}$ model and applied to real radar sea-clutter data, give better performance than those based on the Gaussian model. In [35], the speckling effects on the synthetic aperture radar images have been modeled with the $\mathrm{S} \alpha \mathrm{S}$ distribution.

Moreover, the $\mathrm{S} \alpha \mathrm{S}$ distribution has been used to model heavy tail random processes in image processing and biomedical applications. In $[36,37]$ it has been shown that modeling the data in image watermarking using the $\mathrm{S} \alpha \mathrm{S}$ distribution gives better probability of detection in the discrete cosine transform domain than using the Gaussian distribution. In [38], the $\mathrm{S} \alpha \mathrm{S}$ has been used to model the wavelet coefficients used in image fusion. Also, for target tracking, in [39], it has been shown that modeling of the background fixed objects using the $\mathrm{S} \alpha \mathrm{S}$ distribution gives better probability of detection of the moving target. Moreover, in [40], it has been shown that the frequency spectra of brain magnetic resonance images exhibit non-Gaussian heavy tail behavior and it is better to use the $\mathrm{S} \alpha \mathrm{S}$ model than the Gaussian model. In $[41,42]$, the $S \alpha S$ distribution has been used to model the wavelet coefficients of ultrasonic images. In [43], the S $\alpha \mathrm{S}$ distribution has been used to model non-Gaussian background noise in the evoked potentials that are used to quantify the neurological system properties. 


\subsection{Research Objectives}

When designing a receiver for signals corrupted by additive noise, an optimal receiver is one that minimizes the probability of error. The design of such a receiver must necessarily take into consideration the pdf of the noise. When the noise is Gaussian, the optimal receiver can be simplified to a form with very low complexity. However, when the noise is non-Gaussian, it is not always possible to simplify the receiver while maintaining optimality, so suboptimal yet practical alternatives are considered instead.

The main objective of the proposed work is to design low complexity receivers with near-optimal performance for signals corrupted by non-Gaussian impulsive noise. In particular, the symmetric alpha stable $(\mathrm{S} \alpha \mathrm{S})$ model has been chosen because of its close fit for impulsive noise. The proposed work is concerned with the detection and the decoding functions of the receiver. Moreover, the research results have been also used to design simple suboptimal receivers for signals in Middleton's Class A noise.

\subsubsection{Detection}

The optimal maximum likelihood detector for signals in $\mathrm{S} \alpha \mathrm{S}$ noise requires complex computations, which imposes a challenge in practical implementations. Many suboptimal detectors have been proposed, however, there is always a trade-off between the complexity and the performance. Suboptimal detectors with affordable complexity have poor performance compared to the optimal one. Also, there is no methodology or framework that can be used to explain the performance of these detectors. In this research, we propose a different approach to design low complexity suboptimal detectors by analyzing the behavior of the optimal detector when processing the received samples. The proposed analysis is used to improve the performance of the existent detectors and develop a new low cost near-optimal detector based on a 
simple piecewise linear approximation of the optimal log-likelihood ratio (LLR).

\subsubsection{Decoding}

The design of an optimal decoder for signals that have been encoded with an error correcting code and transmitted over a $\mathrm{S} \alpha \mathrm{S}$ noise channel is a complex task because of the lack of a closed form expression of the S $\alpha \mathrm{S}$ noise pdf. Decoders that are designed based on a Gaussian assumption give poor performance. Many suboptimal designs for different decoders, such as the Viterbi and MAP decoders, have been proposed in the literature. These decoders use simple nonlinearities to enhance the performance of the conventional decoders which are optimized for Gaussian noise. However, their performance is still far from optimal. Moreover, many suboptimal decoders depend on ad hoc methods to enhance the performance. In this thesis, we propose a different approach to design different suboptimal decoders by using a simple approximation of the optimal LLR which results in near-optimal performance with low complexity. The proposed approach is applied to the Viterbi and MAP decoders, but it can also be used with other types of decoders such as the turbo, LDPC and BICM decoders.

\subsubsection{Application to the Class A Noise Model}

The proposed LLR-based framework has been used with the $\mathrm{S} \alpha \mathrm{S}$ noise to design low-complexity detectors and decoders. In the last part of the thesis, we show the generality of the research results by using the proposed LLR-based method to design suboptimal detectors and decoders for the case where the non-Gaussian noise is modeled as Middleton Class A. The resultant detector and Viterbi decoder give near-optimal performance with low complexity. 


\subsection{Research Contributions of the Thesis}

The research contributions of this thesis fall within two areas: detector design for uncoded communication systems and decoder design for systems that employ error correcting codes. With regards to detector design, the main contributions of the thesis are:

1. A novel framework based on decision regions is proposed to analyze the behaviour of optimal and suboptimal detectors. Application of this framework has provided considerable insight into why some suboptimal detectors work well while others do not. This insight in turn led to the design of two novel simple suboptimal detectors with near-optimal performance.

2. To overcome some limitations of the decision-region-based framework for detector design, we proposed an alternative approach based on analysis of the LLR of the received samples. A very simple detector based on using a piecewise linear approximation to the optimal LLR is proposed. This novel detector yields performance nearly indistinguishable from the optimal detector over a wide range of operating conditions.

In addition to these two main contributions, several other contributions of this thesis are:

1. The adaptive soft limiter detector is proposed that is extremely simple and works very well when the number of samples per symbol, $N$, is large and $\alpha$ has moderate to high values.

2. A piecewise linear detector is proposed, which approximates the optimal decision regions by simple linear segments and gives near-optimal performance for the case when $N=2$.

3. As an application to the LLR-based method, the PWL-LLR diversity combiner is proposed, which is a robust simple suboptimal detector that can be used with 
any value of $\alpha$ and $N$ for the case when multiple receive antennas are used with fading channel.

4. To emphasize the generality of the LLR-based approach to detector design, the technique is applied to designing a detector for signals corrupted by Class A noise. A simple piecewise linear approximation to the LLR is presented and the resulting detector is extremely effective.

With regards to decoder design, the contributions of the thesis include:

1. A new approach for decoder design by using the LLR-based framework is proposed. PWL-LLR Viterbi and MAP decoder are proposed to achieve nearoptimal performance with low complexity.

2. The proposed LLR-based method is used with Class A noise which results in a PWL-LLR detector and a PWL-LLR Viterbi decoder.

3. A dual-absolute branch metric for simple Viterbi decoding in $\mathrm{S} \alpha \mathrm{S}$ noise is proposed by using the LLR-approach (implicit use of LLR).

Parts of this thesis appear in the following publications:

T. Shehata Saleh, I. Marsland, and M. El-Tanany, "Suboptimal detectors for alpha-stable noise: Simplifying design and improving performance," Accepted to IEEE Transaction on Communications, February 2012.

T. S. Shehata, I. Marsland, and M. El-Tanany, "A Simplified LLR-Based Detector for Signals in Class-A Noise," Accepted to IEEE Vehicular Technology Conference, Fall 2012.

T. Shehata Saleh, I. Marsland, and M. El-Tanany, "A simplified LLR-based Viterbi decoder for convolutional codes in symmetric alpha-stable noise," Accepted to the Canadian Conference on Electrical and Computer Engineering (CCECE-2012), February 2012.

T. S. Shehata, I. Marsland, and M. El-Tanany, "A low-complexity near-optimal MAP decoder for convolutional codes in symmetric alpha-stable noise," Accepted to the Canadian Conference on Electrical and Computer Engineering (CCECE-2012), February 2012.

T. S. Shehata, I. Marsland, and M. El-Tanany, "A low-complexity near-optimal Viterbi decoder for convolutional codes in Class A noise," Accepted to the Canadian Conference on Electrical and Computer Engineering, Feb. 2012.

T. S. Shehata, I. Marsland, and M. El-Tanany, "Near optimal Viterbi decoders for convolutional codes in symmetric alpha-stable noise," in IEEE Vehicular Technology Conference, Fall 
2010. VTC'10. Sept. 2010.

T. S. Shehata, I. Marsland, and M. El-Tanany, "A novel framework for signal detection in alpha-stable interference," in IEEE Vehicular Technology Conference, Spring 2010. VTC'10. May 2010.

T. S. Shehata, I. Marsland, and M. El-Tanany, "A low complexity piecewise suboptimal detector for signals in alpha-stable interference," in IEEE Vehicular Technology Conference, Spring 2010. VTC'10. May 2010.

One more journal paper is currently being written, and another is planned.

\subsection{Thesis Organization}

In Chapter 2, a background on the $\mathrm{S} \alpha \mathrm{S}$ distribution and its characteristics is presented. In Chapter 3, a survey on the optimal and suboptimal detectors for signals in $\mathrm{S} \alpha \mathrm{S}$ noise is presented. In Chapter 4, the decision regions-based framework is proposed, which is used to design the adaptive soft-limiter and the piecewise linear detector. In Chapter 5, the LLR-based method is proposed, which is used to design the PWL-LLR detector and the PWL-LLR diversity combiner. In Chapter 6 , a survey on the optimal and suboptimal decoders for coded signals in $\mathrm{S} \alpha \mathrm{S}$ noise is presented. In Chapter 7, a new approach for decoder design using the proposed LLR-based framework is proposed, which is used to design PWL-LLR Viterbi and PWL-LLR MAP decoders. In Chapter 8, the LLR-based method is extended to the case of Class A model which is used to design PWL-LLR detector and PWL-LLR Viterbi decoder. In Chapter 9, the conclusion and the future work are presented. 


\section{Chapter 2}

\section{Symmetric Alpha-Stable: A Model for Impulsive Noise}

The stable laws and stable random processes have been intensively discussed in the literature [44-46]. In this chapter, we give a background on the $\mathrm{S} \alpha \mathrm{S}$ distribution and some of its properties that will be useful for the continuity of the context.

\subsection{Properties of the Alpha-Stable Distribution}

One of the properties of the $\alpha$-stable distribution is that there is no closed form expression for the probability density function, except for special cases where $\alpha=1$ (Cauchy distribution) and $\alpha=2$ (Gaussian distribution). Instead, the $\alpha$-stable distribution is described by its characteristic function:

$$
\phi_{X}(\omega)=\exp \left\{j \delta \omega-\gamma|\omega|^{\alpha}(1-j \beta \operatorname{sgn}(\omega) \theta(\omega, \alpha))\right\}
$$

where

$$
\theta(\omega, \alpha)=\left\{\begin{array}{cc}
\tan \left(\frac{\alpha \pi}{2}\right) & \alpha \neq 1 \\
-\frac{2}{\pi} \log |\omega| & \alpha=1
\end{array}\right.
$$


and

$$
\operatorname{sgn}(\omega)=\left\{\begin{array}{cc}
1 & \omega>0 \\
0 & \omega=0 \\
-1 & \omega<0
\end{array}\right.
$$

The stable distribution is completely defined by four parameters: $\alpha, \gamma, \delta$ and $\beta$, where $\alpha \in(0,2]$ is the characteristic exponent, which measures the thickness of the distribution's tail (a small value of $\alpha$ implies considerable probability mass in the tails, such that it is likely to have noise values far from the distribution's center); $\gamma>0$ is the noise dispersion, which measures the distribution's spread around its center; $\beta \in[-1,+1]$ is the symmetry parameter which controls the skewness of the stable distribution about the location parameter, $\delta$. When $\beta= \pm 1$, the stable distribution is called totally skewed, and when $\beta=0$, the distribution becomes symmetric around $\delta$. In this case, the distribution is called symmetric $\alpha$-stable $(\mathrm{S} \alpha \mathrm{S})$. For $\mathrm{S} \alpha \mathrm{S}$ distributions, $\delta$ represents the mean when $1<\alpha \leq 2$ and the median when $0<\alpha \leq 1$. Because of the symmetry of the non-Gaussian noise in many applications, we consider the class of symmetric $\alpha$-stable with zero location parameter $(\delta=0)$. The characteristic function of the zero-mean $\mathrm{S} \alpha \mathrm{S}$ distribution reduces to

$$
\phi_{X}(\omega)=e^{-\gamma|\omega|^{\alpha}}
$$

The pdf of the S $\alpha \mathrm{S}$ distribution, $f_{\alpha}(x)$, is given by the inverse Fourier transform of (2.4). Although no closed form expression is known for the resulting pdf, it can be evaluated numerically. Figure 2.1 shows the probability density function for different values of $\alpha$. It can be shown that the $\mathrm{S} \alpha \mathrm{S}$ distribution is smooth and has a bell shape like the Gaussian distribution, however, it has heavier tails than the Gaussian distribution $(\alpha=2)$ as shown in Figure 2.2. Different random realizations of a white $\mathrm{S} \alpha \mathrm{S}$ process for different values of $\alpha$ are shown in Figure 2.3. 


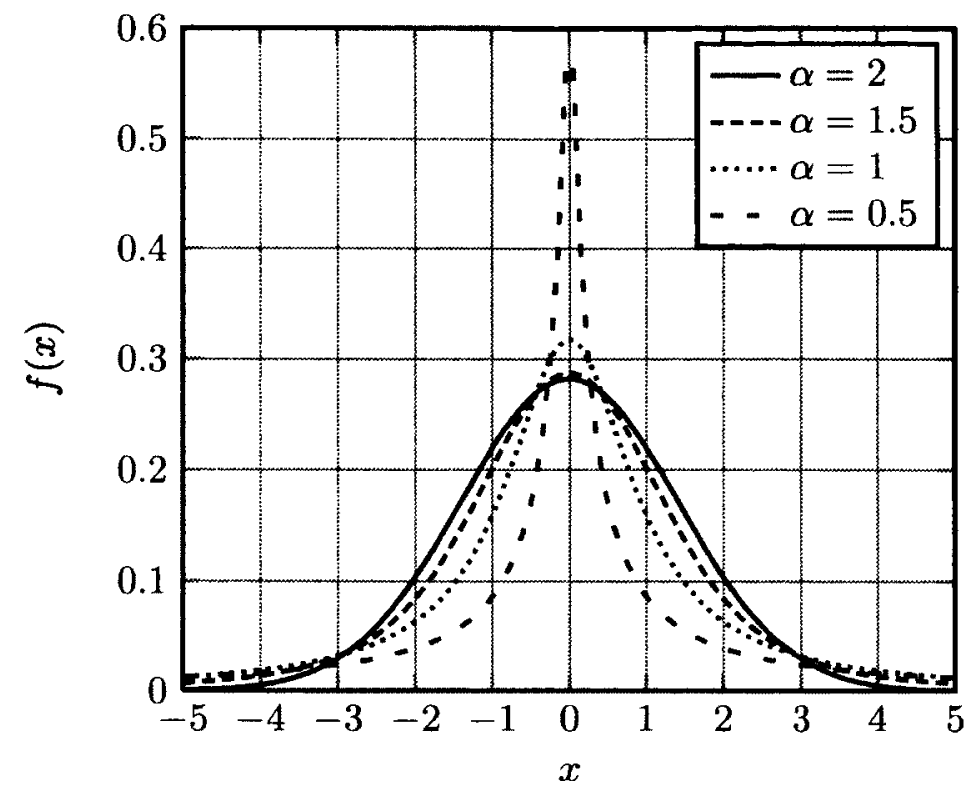

Figure 2.1: Symmetric $\alpha$-stable distribution with $\gamma=1$.

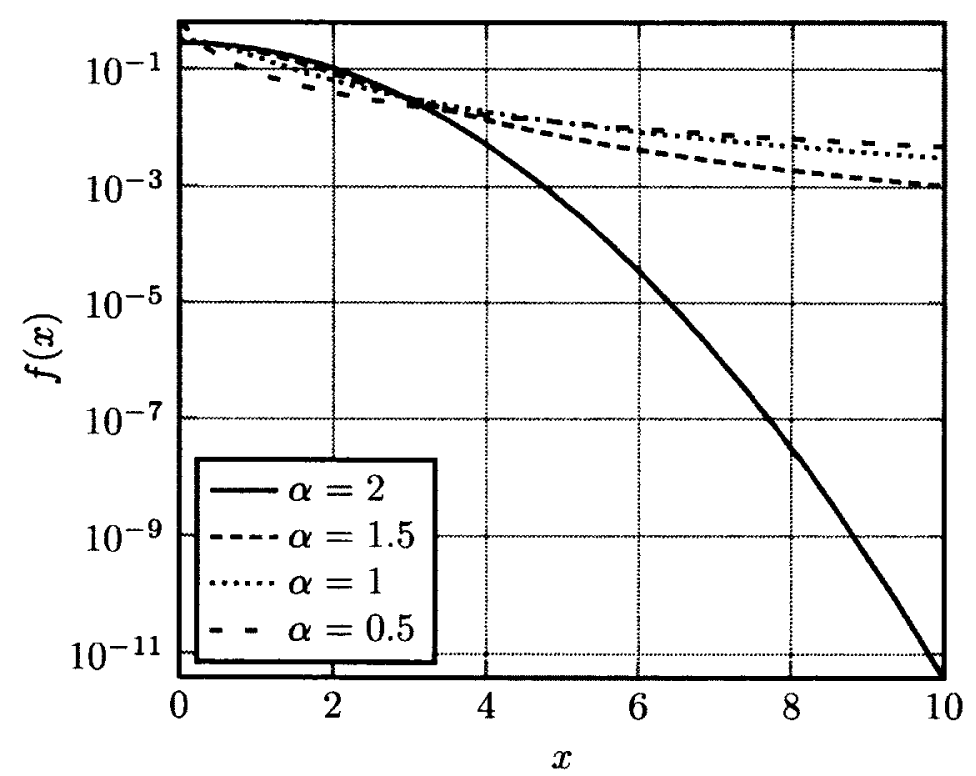

Figure 2.2: Symmetric $\alpha$-stable distribution with $\gamma=1$ on $\log$ scale. 


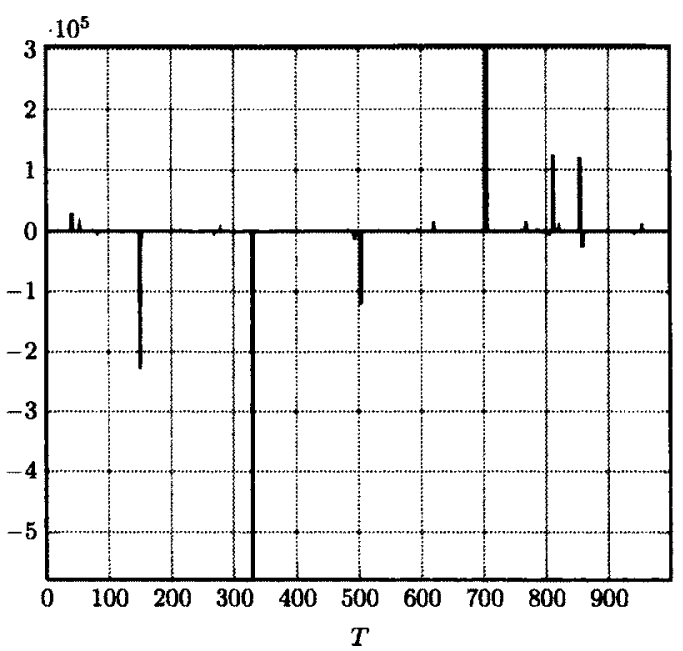

(a) $\alpha=0.5$

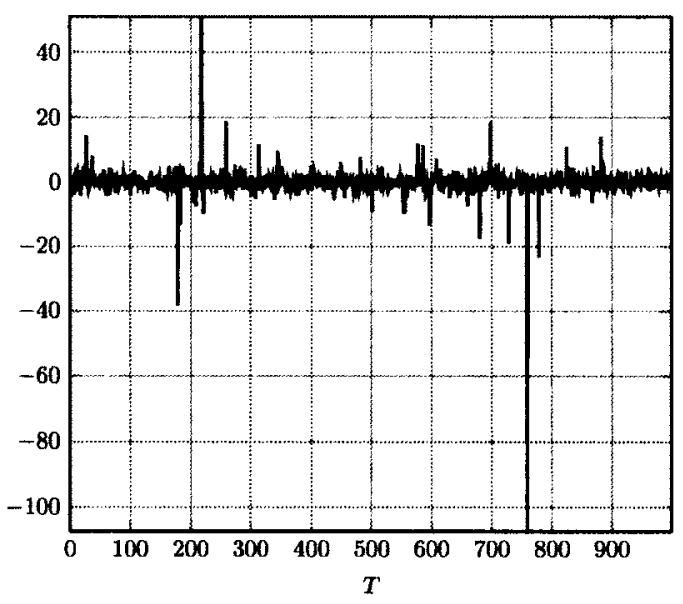

(c) $\alpha=1.5$

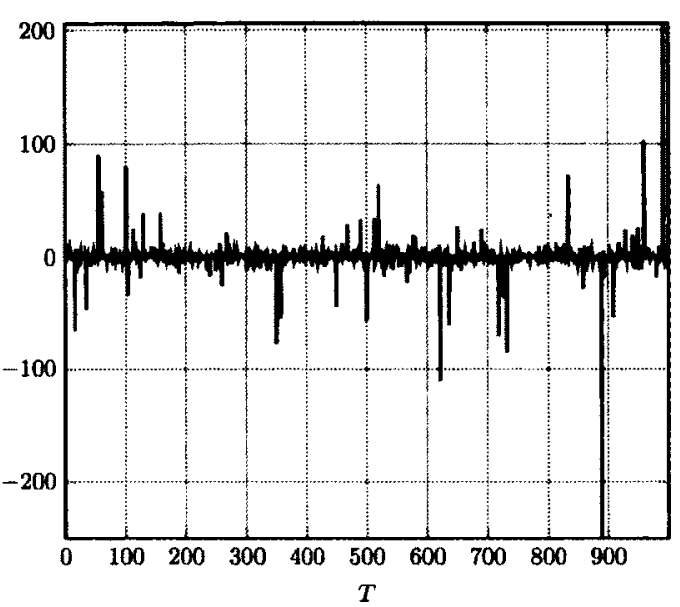

(b) $\alpha=1$

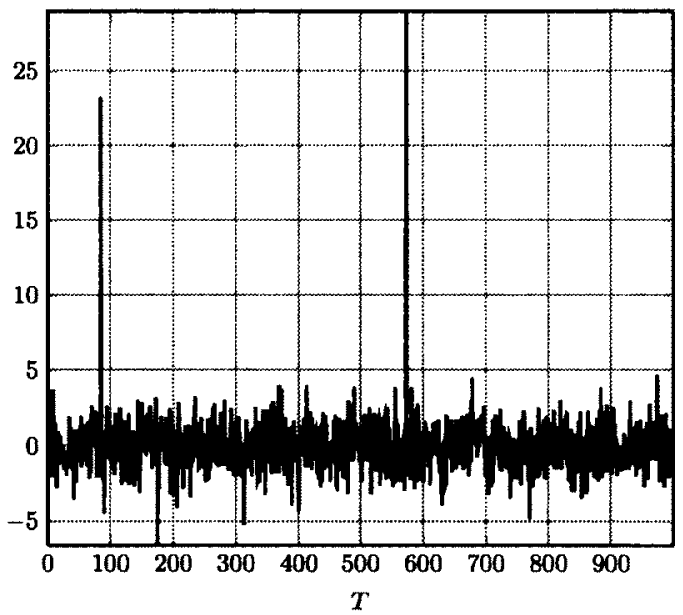

(d) $\alpha=1.9$

Figure 2.3: Realizations of symmetric $\alpha$-stable processes for different values of $\alpha$, with $\gamma=1$.

We will use the notation $X \sim S_{\alpha}(\delta, \gamma)$ to represent the $\mathrm{S} \alpha \mathrm{S}$ random variable (RV) $X$ with location parameter $\delta$, noise dispersion $\gamma$, and characteristic exponent $\alpha$. Some properties of $\mathbf{S} \alpha \mathrm{S}$ random variables [8]

1. Let $X=X_{1}+X_{2}$, where $X_{1} \sim S_{\alpha}\left(\delta_{1}, \gamma_{1}\right)$ and $X_{2} \sim S_{\alpha}\left(\delta_{2}, \gamma_{2}\right)$ be independent 
S $\alpha$ S RVs. Then, $X \sim S_{\alpha}\left(\delta_{1}+\delta_{2}, \gamma_{1}+\gamma_{2}\right)$.

2. If $X \sim S_{\alpha}(\delta, \gamma)$ and let $h$ be a real number. Then $h X \sim S_{\alpha}\left(h \delta,|h|^{\alpha} \gamma\right)$.

3. If $X \sim S_{\alpha}(0, \gamma)$ and $\alpha \neq 2$, then

$$
\lim _{x \rightarrow \infty} \operatorname{Pr}(X>x) \sim \gamma \frac{C_{\alpha}}{2} x^{-\alpha}
$$

where

$$
C_{\alpha}= \begin{cases}\frac{2}{\pi} & \alpha=1 \\ \frac{1-\alpha}{\Gamma(2-\alpha) \cos (\pi \alpha / 2)} & \alpha \neq 1\end{cases}
$$

and $\Gamma(\cdot)$ is the Gamma function. This property describes the polynomial asymptotic behavior of the decaying of the $\alpha$-stable distribution. For the Gaussian case $(\alpha=2)$, the asymptotic behavior of the tail is exponential [47]

$$
\lim _{x \rightarrow \infty} \operatorname{Pr}(X>x) \sim \frac{1}{2 \sqrt{\pi} \sigma x} \exp \left\{-\frac{x^{2}}{4 \sigma^{2}}\right\}
$$

where $\sigma^{2}=2 \gamma$ is the variance of $X$.

\subsection{Definition of Signal-to-Noise Ratio in The Context of $\mathrm{S} \alpha \mathrm{S}$ Noise}

The $\mathrm{S} \alpha \mathrm{S}$ random variable possess a finite absolute moment only for orders less than $\alpha$; i.e.,

$$
E\left[|X|^{p}\right]<\infty \quad \text { iff } \quad p<\alpha, 0<\alpha<2
$$

This property implies that the power of the S $\alpha \mathrm{S}$ process becomes infinite because it depends on the second order moment of the S $\alpha \mathrm{S} \mathrm{RV}$, so the standard signal to noise ratio becomes meaningless. Two common approaches exist to measure the strength 
of the $\mathrm{S} \alpha \mathrm{S}$ random process and consequently the signal-to-noise ratio.

- The signal power to noise dispersion ratio

The dispersion of the noise distribution, which is a measure of the spread around the center, is used as an indicator for the random process strength [10] [48]. The signal to noise dispersion ratio is defined as:

$$
\operatorname{SNDR}=\frac{E_{s}}{4 \gamma}
$$

where $E_{s}$ is the received energy per symbol and $\gamma$ is the noise dispersion. The scale factor of 4 is used such that the SNDR reduces to $\frac{E_{s}}{N_{o}}$ when $\alpha=2$ (Gaussian noise) where the noise variance is defined as $2 \gamma$.

\section{- The geometric signal power to geometric noise power ratio}

The geometric signal power was proposed in [49] as a better indicator of the strength of the heavy-tail processes. It is based on the geometric mean:

$$
S_{0}(X)=e^{E[\log |X|]}
$$

and it was proven that $S_{0}^{2}$ is a power indicator that can be used to describe and compare the strength of different processes. The geometric signal-to-noise ratio (GSNR) is defined as [49]:

$$
\mathrm{GSNR}=\frac{1}{4 C_{g}}\left(\frac{E_{s}}{S_{0}^{2}}\right)
$$

where $C_{g}=e^{C_{e}} \approx 1.78$ is the exponential of Euler's constant and $S_{0}^{2}$ is the geometric power of a $S \alpha S$ random variable, where $S_{0}=C_{g}^{\frac{1}{\alpha}-1} \gamma^{\frac{1}{\alpha}}$. The scale factor $\frac{1}{4 C_{g}}$ is used such that the GSNR reduces to the standard SNR in case of $\alpha=2$ (Gaussian noise). 
In this thesis, we use the geometric signal power to geometric noise power ratio (GSNR) to measure the relative signal to noise strength.

\subsection{System Model}

In this thesis, we use the same channel model used in $[10,48,50,51]$. A sequence of BPSK symbols $\left\{s_{i} \in\{ \pm A\}\right\}$ is transmitted, and $N$ independent samples per symbol are collected at the receiver, such as when oversampling is used $[8,22,48,50]$ or when $N$ independent multipath channels or multiple receive antennas are used $[8,22]$ or when bit repetition is used [32]. The received samples are modeled as

$$
r_{i k}=s_{i}+n_{i k} \quad k=1, \ldots, N
$$

where, for each $i,\left\{n_{k}\right\}$ are $N$ independent $\mathrm{S} \alpha \mathrm{S}$ noise samples collected during the symbol duration $T$. We will use the assumption of the collecting $N$ independent samples per symbol particularly in Chapters 3,4 and 5 where the detector analysis is proposed. In Chapters 6, we will use only one sample per symbol $(N=1)$. 


\section{Chapter 3}

\section{Signal Detection in SaS Noise:}

\section{Background and Literature Review}

\subsection{Introduction}

In this chapter, we give a comprehensive background on the detector design for binary signals in $\mathrm{S} \alpha \mathrm{S}$ noise. We review the optimal and several suboptimal detectors in the literature such as the Cauchy detector, the Myriad filter, the Gaussian (linear) detector, the soft limiter (SL) detector, the locally optimal Baysian (LOB) detector and the sign correlator (SC) detector. The performance of the suboptimal detectors is compared with that of the optimal one in terms of the probability of bit error $\left(P_{e}\right)$. The conclusion drawn from the performance comparison leads to some important questions which drive the main contributions of the detection part of the thesis. We use the channel model presented in Section 2.3 where the received samples are modeled as:

$$
r_{i k}=s_{i}+n_{i k} \quad k=1, \ldots, N
$$

where, for each $i, s_{i} \in\{ \pm A\}$ and $\left\{n_{i k}\right\}$ are $N$ independent $S \alpha S$ noise samples per received symbol. For simplicity, we will drop the index $i$ and consider the samples received within the interval of only one symbol. 


\subsection{Optimal Maximum Likelihood Detector}

The optimal maximum likelihood (ML) detector computes the following test statistic for a received symbol:

$$
\lambda_{M L}=\sum_{k=1}^{N} \log \left\{\frac{f_{\alpha}\left(r_{k}-A\right)}{f_{\alpha}\left(r_{k}+A\right)}\right\} \stackrel{s_{1}}{\sum_{s_{0}}} 0
$$

where $s_{0}$ is the hypothesis that $s=-A$ was transmitted, and $s_{1}$ is the hypothesis that $s=+A$ was transmitted. The ML detector is impractical because it requires complex computations such as numerical integration or DFT operation to calculate the probability density function for each received sample.

Complexity of the test statistic:

- $N \log$ operations.

- $N$ Division operations.

- $2 N$ L-point-FFT operations.

- Knowledge of $\alpha, \gamma$, and $A$.

For a single FFT operation, a tight lower bound of the number of real multiplications is $O L$, where $L$ is the number of the point of the FFT operation, and the number of real addition is $L \log _{2} L$ [52].

The implementation of the log operation is usually approximated by Taylor series

$$
\log x=2 \sum_{n=1}^{M} \frac{((x-1) /(x+1))^{2 n-1}}{2 n-1} \quad(x>0)
$$

which requires $\sum_{n=2}^{L} 2 n$ multiplications and one summation. 


\subsection{Suboptimal Detectors}

\subsubsection{Cauchy Detector}

The ML optimal detector can be simplified assuming $\alpha=1$ in (3.2), which results in the Cauchy detector. For the case when $\alpha=1$, the probability density function has a closed form expression

$$
f\left(r_{k} \mid s\right)=\frac{\gamma}{\pi} \frac{1}{\gamma^{2}+\left(r_{k}-s\right)^{2}}
$$

The test statistic for the Cauchy detector is

$$
\lambda_{\text {Cauchy }}=\sum_{k=1}^{N} \log \left[\frac{\gamma^{2}+\left(r_{k}+A\right)^{2}}{\gamma^{2}+\left(r_{k}-A\right)^{2}}\right]
$$

The Cauchy detector has been used as a suboptimal detector for all values of $\alpha$ because of its robust performance against $\alpha[8,50]$, but it is still complex because of the need to calculate the log. However, there is no clear explanation for the robustness of the Cauchy detector. In [53], it was reported that the performance gain, which the Cauchy detector has over many suboptimal detectors, is due to the dependence of the test statistics on the noise dispersion $\gamma$; however, this justification was not proven and also it does not depend on clear criteria to justify the performance.

Complexity of the test statistic:

- $N \log$ operations.

- $N$ division operations.

- $2 N$ multiplications.

- $2 N$ additions.

- Knowledge of $\gamma$, and $A$. 


\subsubsection{Myriad Filter}

The test statistic of the myriad filter is

$$
\lambda_{\text {Myriad }}=\sum_{k=1}^{N} \log \left[\frac{\kappa^{2}+\left(r_{k}+A\right)^{2}}{\kappa^{2}+\left(r_{k}-A\right)^{2}}\right]
$$

where $\kappa$ is the linearity parameter of the myriad filter. It can be seen that the test statistic of the myriad filter is similar to that of the Cauchy detector except for $\kappa$, which is function of $\gamma$ and $\alpha$. The linearity parameter, $\kappa$, is used to adapt the myriad filter to give better performance for different values of $\alpha$. By fitting experimental data, $\kappa$ was proposed in [54] to be

$$
\kappa=\gamma^{\frac{1}{\alpha}} \sqrt{\frac{\alpha}{2-\alpha}}
$$

which reduces to $\gamma$ when $\alpha=1$. However, the myriad filter is still complex, and it also depends on the robustness of the Cauchy detector which still has no justification.

\subsubsection{Gaussian Detector}

As the simplest suboptimal solution, the ML detector is simplified assuming $\alpha=2$ in (3.2) (i.e., the noise is assumed to be Gaussian) $[8,50]$. The resultant detector is the conventional Gaussian (linear) detector with the following test statistic:

$$
\lambda_{\text {Gauss }}=\sum_{k=1}^{N} r_{k}
$$

However, the Gaussian detector gives poor performance compared to the optimal one when $\alpha<2$. Also, there is no adequate justification for the performance degradation of the Gaussian detector compared to the performance robustness of the Cauchy detector even when $\alpha$ approaches 2 . 


\section{Complexity of the test statistic:}

- 1 Summation.

\subsubsection{Soft Limiter (SL) Detector}

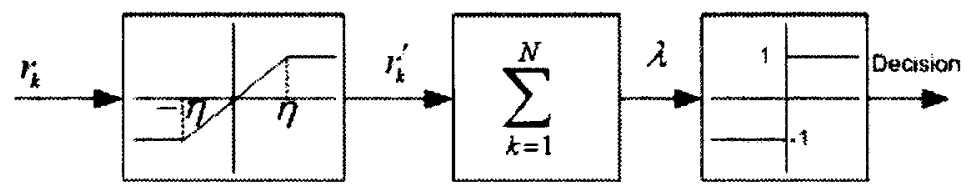

Figure 3.1: Block diagram of the soft limiter detector

As an attempt to improve the performance of the Gaussian detector, a soft limiter (SL) detector was introduced $[8,31,48,50]$, in which a clipping device is used to clip the received samples to a certain threshold, $\eta$, before the Gaussian detector as shown in Figure 3.1. The use of the clipping device was motivated because it increases the GSNR at the input of the Gaussian detector [50]. However, as will be shown later, this is not the reason why the SL detector usually improves the performance over the Gaussian detector. Also, arbitrary fixed threshold values were used [50]. In [31] and [48], it was argued that the optimal threshold value should minimize the probability of error. However, no clear method to calculate the threshold was provided, and the probability of error was calculated assuming a locally optimal nonlinearity was applied to the received samples, even though the limiter does not even crudely approximate the locally optimal nonlinearity.

\section{Complexity of the test statistic:}

- 1 Summation.

- 1 comparator. 


\subsubsection{Locally Optimal Bayesian (LOB) Detector}

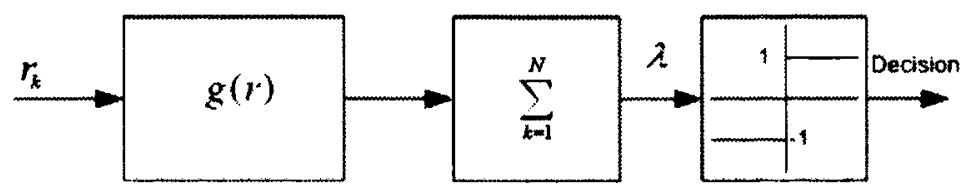

Figure 3.2: Block diagram of the locally optimal detector

The locally optimal Bayesian (LOB) detector is another type of suboptimal detector which uses the locally optimal detection criteria [55] to design an optimal nonlinearity that is applied to the received signal before the Gaussian detector [48] as shown in Figure 3.2. In [55], it was shown that the optimal Bayesian detector can be approximated by the locally optimal nonlinearity for the case of weak signals. By rewriting the test statistic of the optimal detector in (3.2),

$$
\lambda_{M L}=\sum_{k=1}^{N} \log \left[f_{\alpha}\left(r_{k}-A\right)\right]-\log \left[f_{\alpha}\left(r_{k}+A\right)\right]
$$

a Taylor series expansion can be applied for $\log [f(x-u)]$ about $u=0$, which results in

$$
\lambda_{M L}=\sum_{k=1}^{N}(A-(-A)) g_{L O}\left(r_{k}\right)+\frac{1}{2} \sum_{k=1}^{N}\left(A^{2}-\left(-A^{2}\right)\right)\left[\frac{f_{\alpha}^{\prime \prime}\left(r_{k}\right)}{f_{\alpha}\left(r_{k}\right)}-g_{L O}^{2}\left(r_{k}\right)\right]+\cdots
$$

where $g_{L O}(x)$ is the locally optimal nonlinearity [55],

$$
g_{L O}(x)=-\frac{f_{\alpha}^{\prime}(x)}{f_{\alpha}(x)}
$$

In the case of vanishing signal strength, it can be shown that the optimal Baysian detector can be approximated by using the first term of the Taylor series which is the locally optimal nonlinearity, so $\lambda_{L O}=\sum_{k=1}^{N} g_{L O}\left(r_{k}\right)$. However, the locally optimal nonlinearity depends on the probability density function and its first derivative, and 


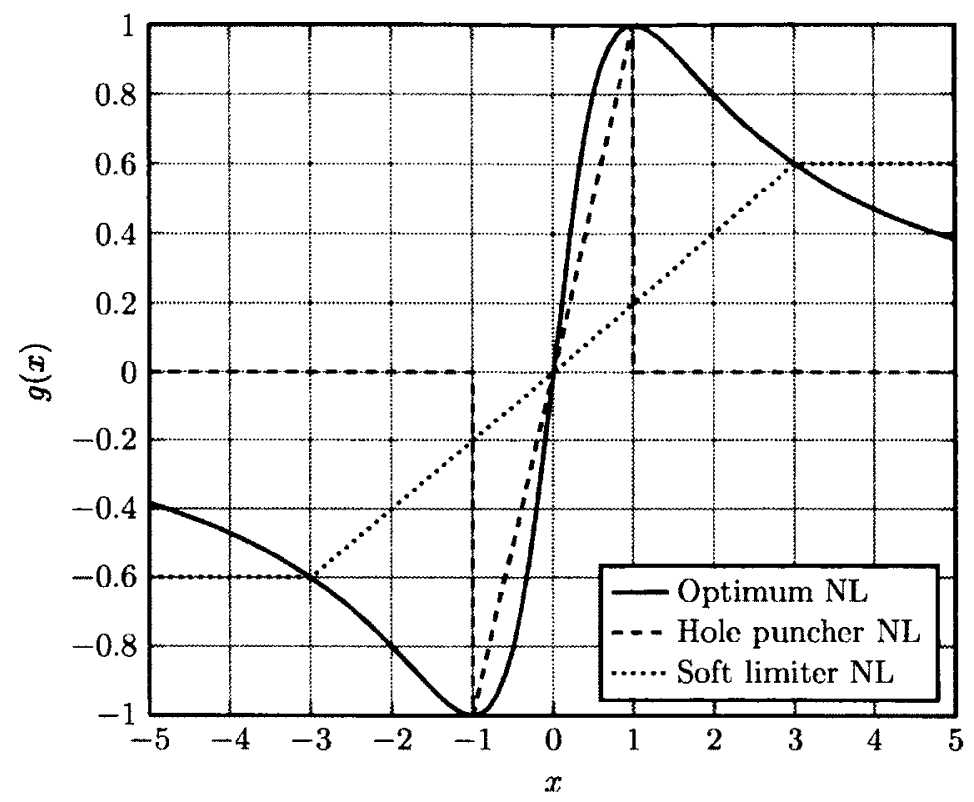

Figure 3.3: Locally optimal nonlinearity with $\alpha=1, \gamma=1$.

therefore it is complex to implement. Many approximations to the locally optimal nonlinearity were proposed such as the hole puncher detector [48] which uses a simple linear approximation as shown in Figure 3.3. Other nonlinear approximations were also used, such as the Gaussian-tailed nonlinearity $[51,56]$, and the finite Gaussian mixture [22]. However, the main limitation of using the locally optimal detector is that it depends on the locally optimal detection criteria which is valid for weak signals only. So, the performance is expected to be good at low GSNR values only.

\subsubsection{Sign Correlator (SC) Detector}

The sign correlator (SC) was introduced in [55] as the locally optimal detector for Laplacian (double exponential) noise with pdf

$$
f(x)=\frac{a}{2} e^{-a|x|} \quad a>0 .
$$


The test statistic is

$$
\lambda_{S C}=\sum_{k=1}^{N} \operatorname{sgn}\left(r_{k}\right)
$$

where

$$
\operatorname{sgn}(x)= \begin{cases}1 & x>0 \\ 0 & x=0 \\ -1 & x<0\end{cases}
$$

The SC detector was used as a simple nonparametric detector for signals in nonGaussian noise in general. It gives surprisingly good performance compared to the optimal one, when $N$ is odd or large as will be explained later.

Complexity of the test statistic:

- 1 Summation.

- 1 comparator.

Although these suboptimal detectors give different levels of performance, there has been very little explanation for why their performance differs. That is, there is no clear definition of what properties a good suboptimal detector should possess, which makes it difficult to design better detectors, other than by trial-and-error.

\subsection{Performance Comparison and Discussion}

\subsubsection{Verification of Simulation}

'Due to the lack of a closed form analytic solution for the BER of the optimum detector, and before we proceed with different simulation setup, we reproduced the simulation results published in [50] to verify our simulation settings. We reproduced the performance of the optimal detector with $N=10$ for different values of $\gamma$. In Figure 3.4 , it can be shown that the simulation result is similar to that published 
in $[50]^{1}$.

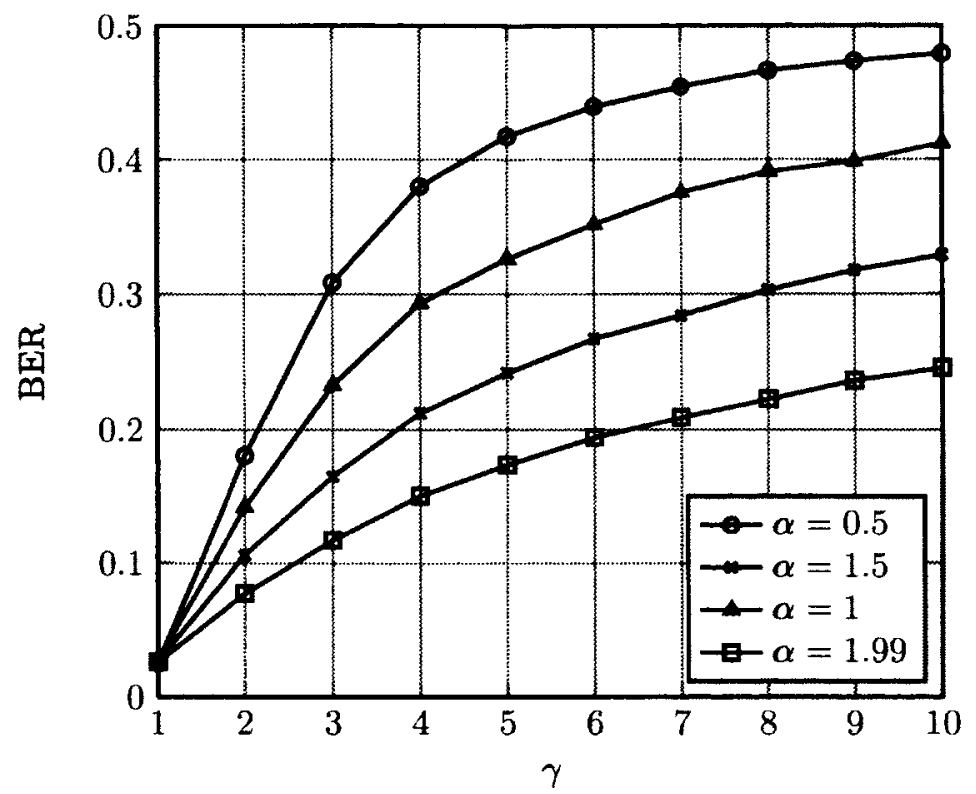

Figure 3.4: BER for the optimal detector for different $\gamma$ (with $N=10$ ). Reproduced results from [50], Figure 5-a.

\subsubsection{Simulation Setup}

To compare the performance, the probability of error $\left(P_{e}\right)$ of the optimal and suboptimal detectors is evaluated by simulation for the cases when $\alpha=1,1.3,1.5,1.7$ and 1.9 with different number of samples per symbol $N=2,3,4,5$ and 10 . Because the noise variance is not defined for values of $\alpha<2$, the geometric signal-to-noise ratio (GSNR), $\frac{1}{4 C_{g}}\left(\frac{E_{s}}{S_{0}^{2}}\right)$, is used (as shown in (2.11)), where $E_{s}=N A^{2}$ is the transmitted energy per symbol, and $S_{0}^{2}=C_{g}^{\frac{2}{\alpha}-2} \gamma^{\frac{2}{\alpha}}$ is the geometric power of the $\mathrm{S} \alpha \mathrm{S}$ random variable and $C_{g} \approx 1.78$ is the exponential of Euler's constant [49]. During the simulation, $\gamma$ has a fixed value while $A$ is changed with different values of GSNR. The receiver is assumed to collect $N$ independent samples per symbol. To calculate an accurate $P_{e}$, up to $4 \times 10^{5}$ frames with 250 bits each are transmitted, and the simulation is

\footnotetext{
${ }^{1}$ Figure 5 -a in reference $[50]$
} 
stopped when the number of bit errors reaches $10^{4}$ or $P_{e} \leq 10^{-5}$ is achieved.

\subsubsection{Performance of the Optimal Detector}

Figures 3.5 to 3.9 show the performance of the optimal detector with different values of $\alpha$ and $N$. It can be shown that for a given value of $\alpha, P_{e}$ decreases when $N$ increases. Figures 3.10 to 3.13 show how the performance is changing with $\alpha$ for a given value of $N$. In general, for higher values of GSNR, $P_{e}$ decreases when $\alpha$ increases.

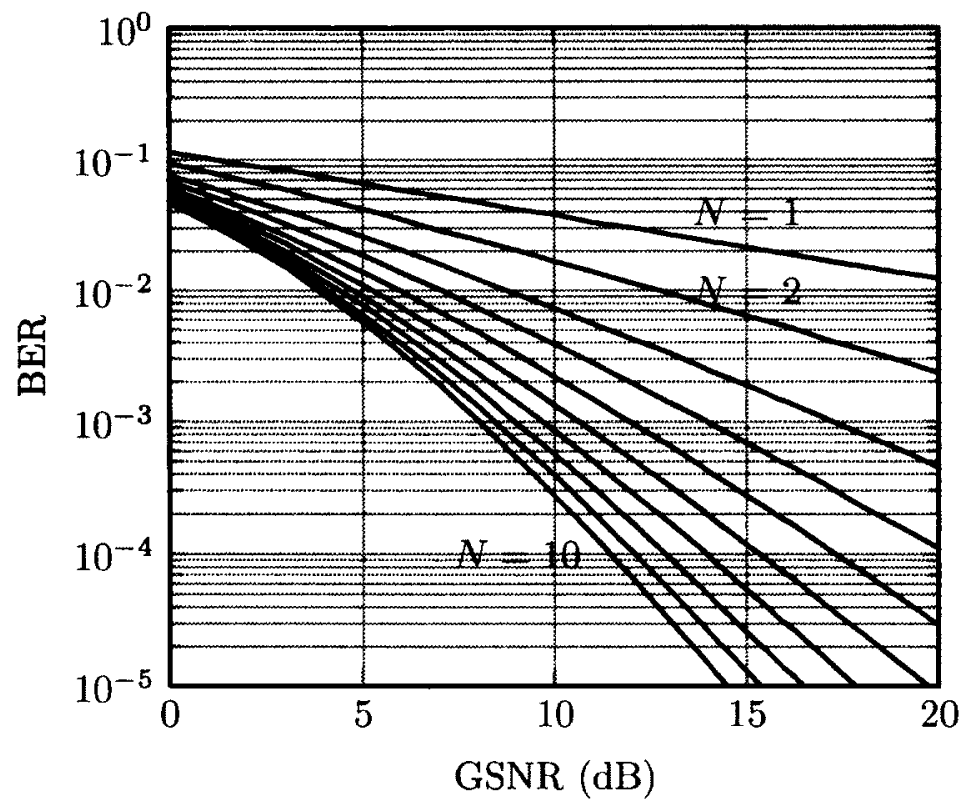

Figure 3.5: BER for the optimal detector for different $N$ (with $\alpha=1$ ). 


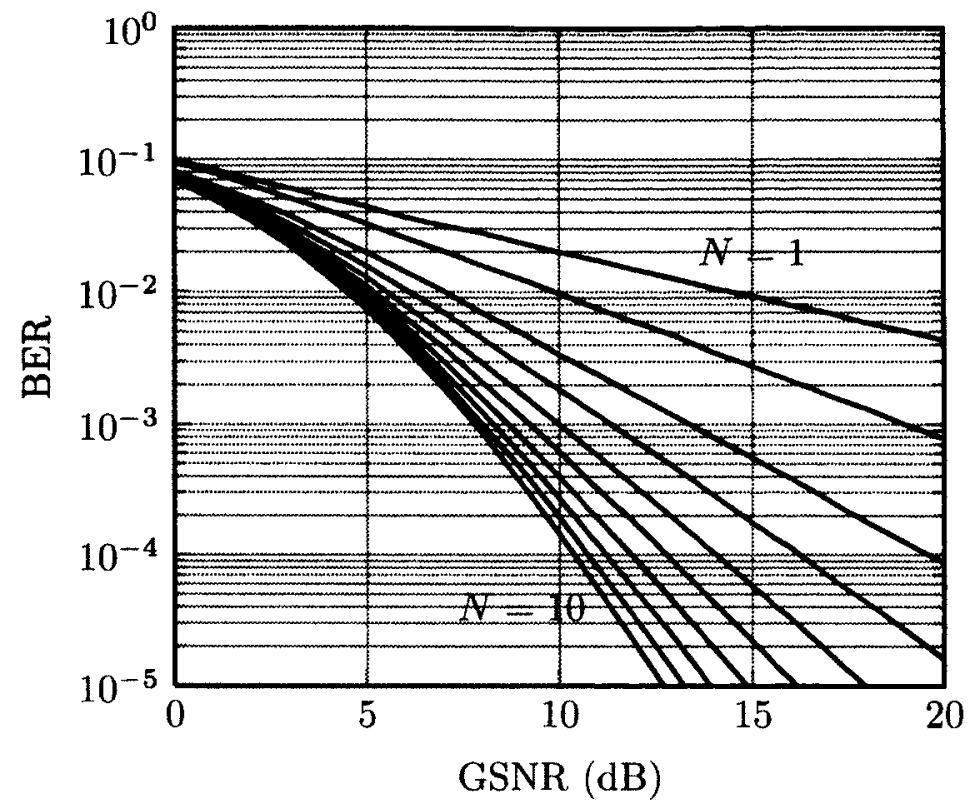

Figure 3.6: BER for the optimal detector for different $N$ (with $\alpha=1.3$ ).

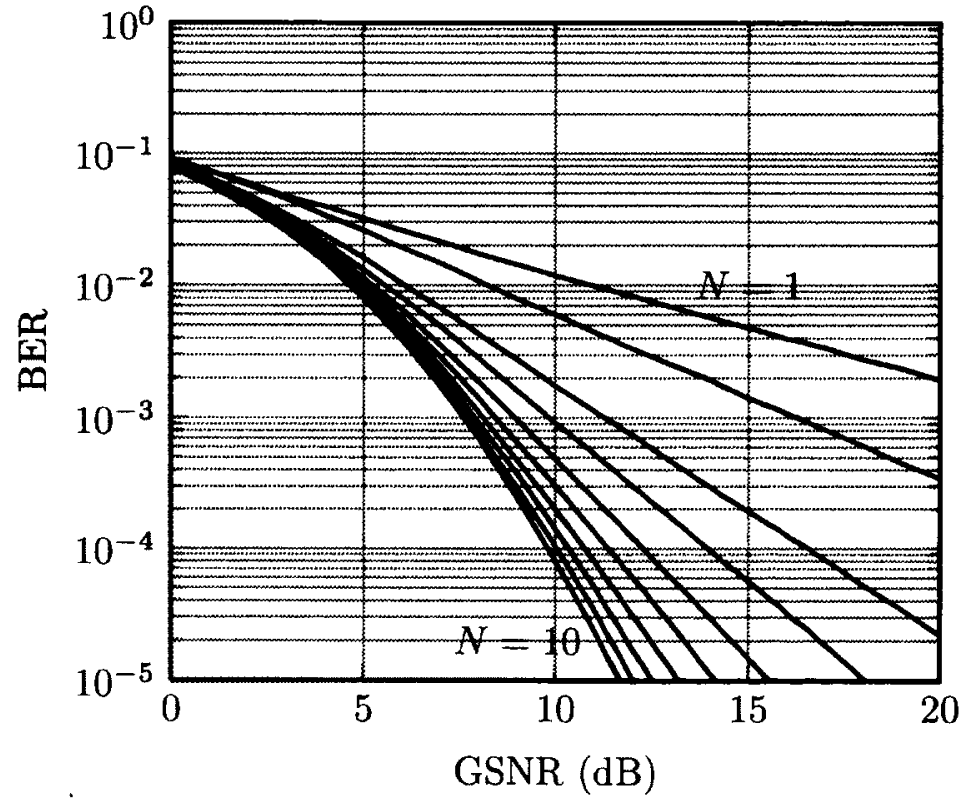

Figure 3.7: BER for the optimal detector for different $N$ (with $\alpha=1.5$ ). 


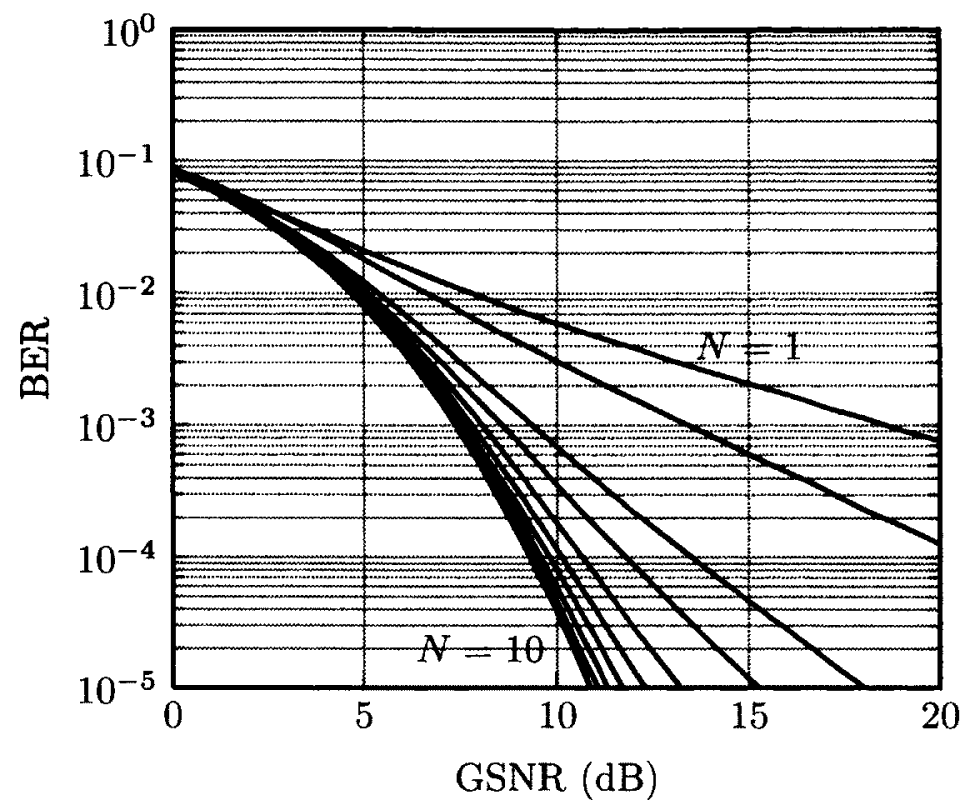

Figure 3.8: BER for the optimal detector for different $N$ (with $\alpha=1.7$ ).

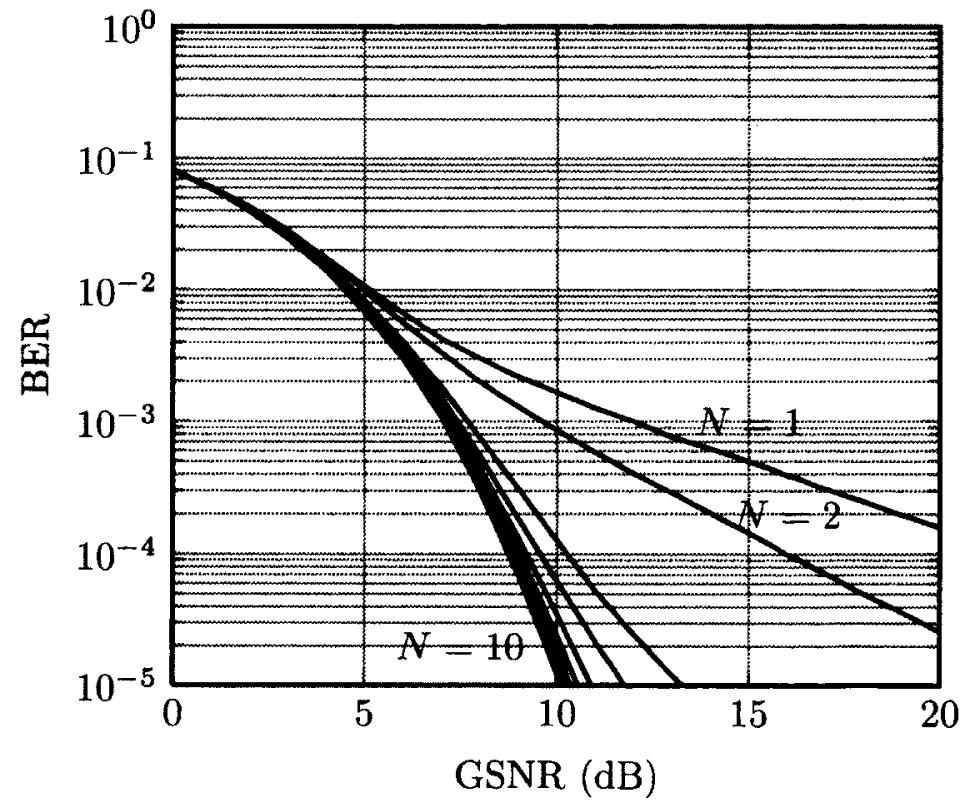

Figure 3.9: BER for the optimal detector for different $N$ (with $\alpha=1.9$ ). 


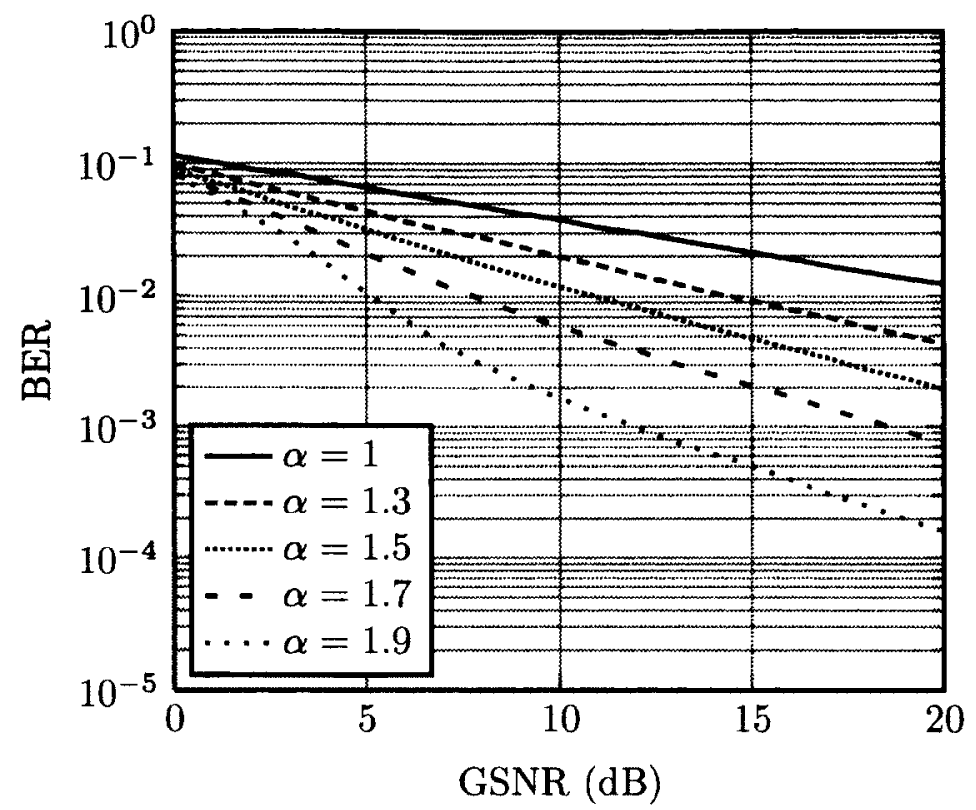

Figure 3.10: BER for the optimal detector for different $\alpha$ (with $N=1$ ).

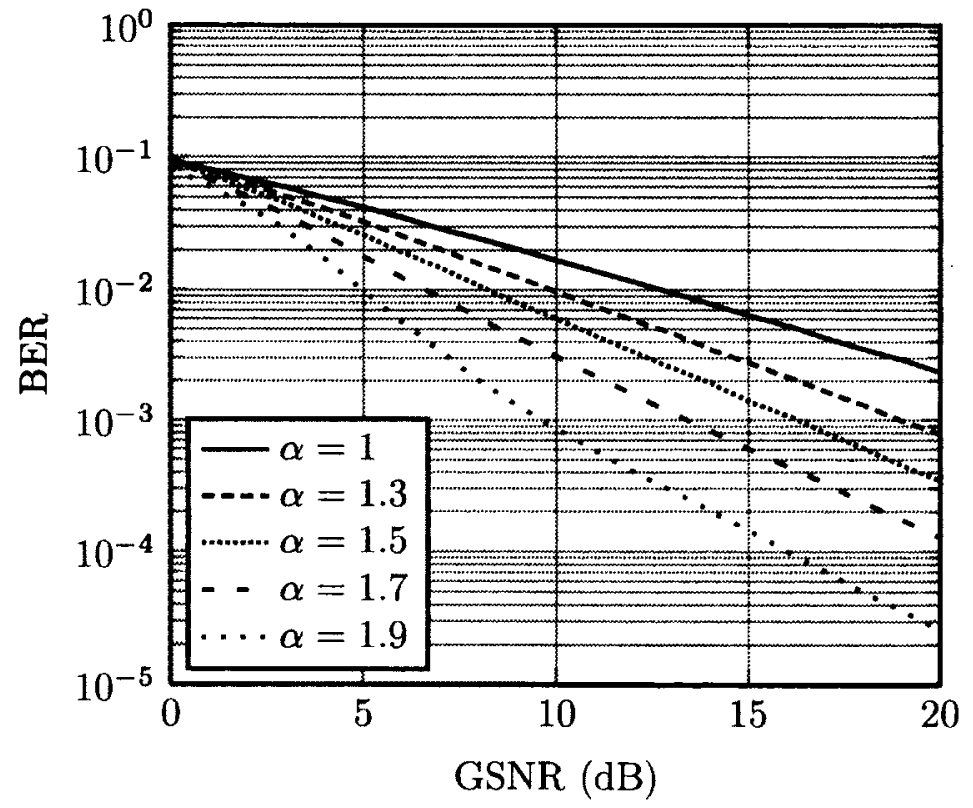

Figure 3.11: BER for the optimal detector for different $\alpha$ (with $N=2$ ). 


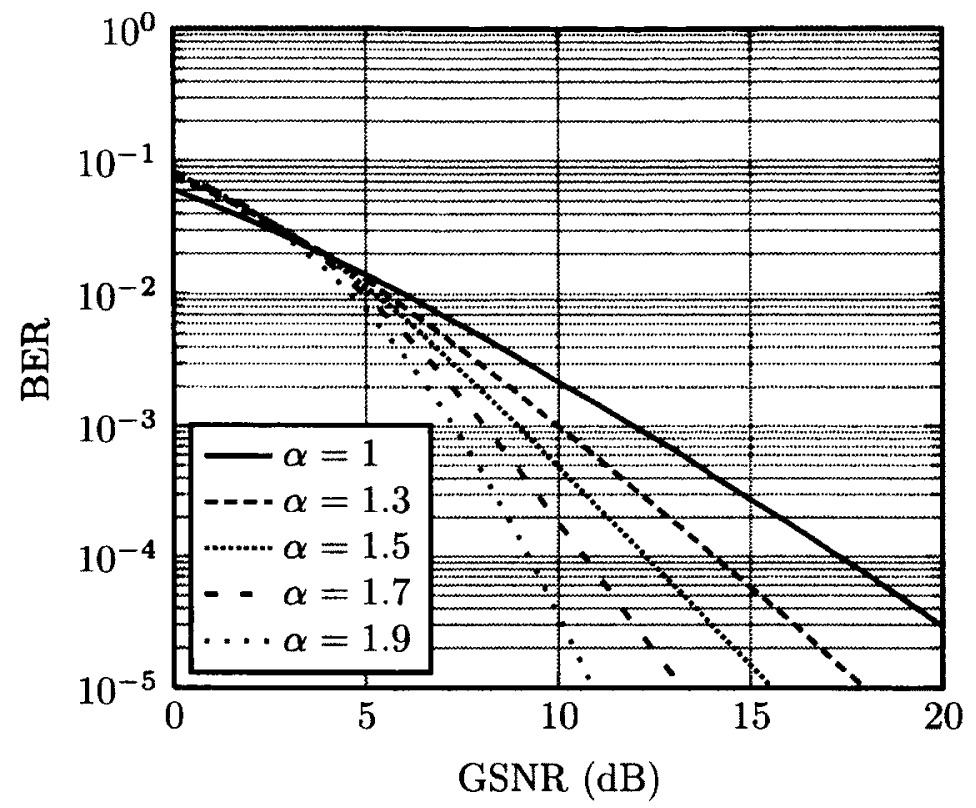

Figure 3.12: BER for the optimal detector for different $\alpha$ (with $N=5$ ).

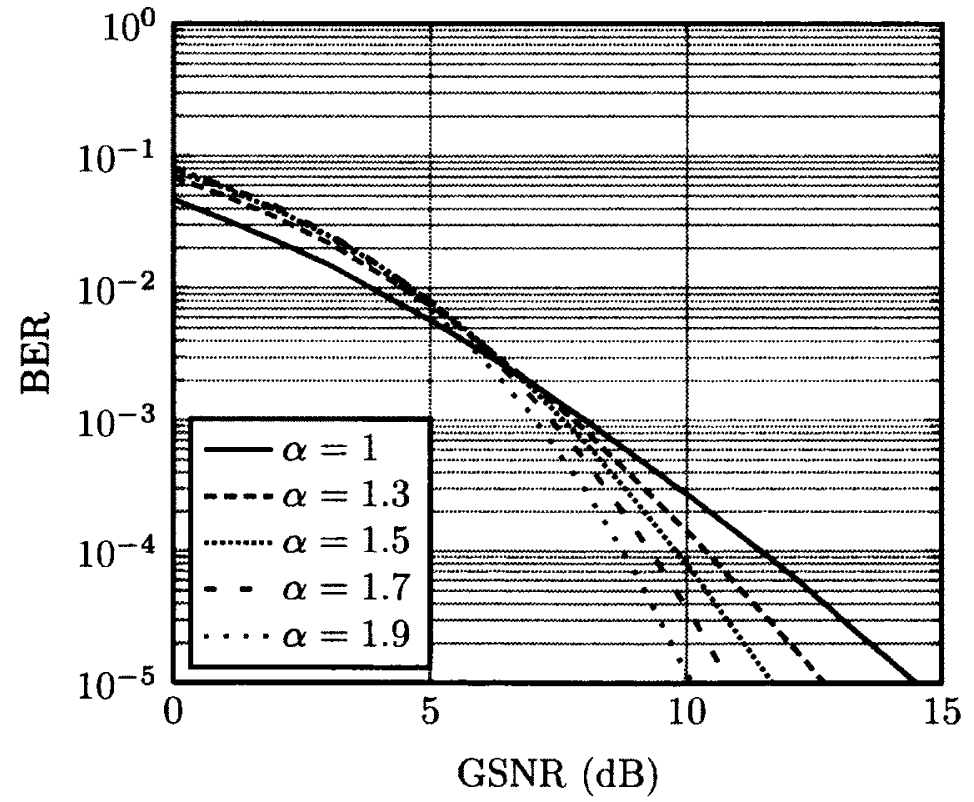

Figure 3.13: BER for the optimal detector for different $\alpha$ (with $N=10$ ). 


\subsubsection{Performance of the Suboptimal Detectors}

The performance of various suboptimal detectors has been evaluated by means of extensive computer simulation for $\alpha=1,1.3,1.5,1.7$ and 1.9 and for $N=2,3,4,5$ and 10. In this section, we will highlight the most important observations.

Figure 3.14 shows the performance of several suboptimal detectors for the case when $\alpha=1.5$ and $N=4$. We can see that both the Cauchy and myriad filter detectors perform very well, nearly matching the performance of the optimal detector. The Gaussian detector, on the other hand, performs quite poorly, with the performance degradation increasing with GSNR. The LOB detector performs slightly better than the Gaussian detector at low GSNR, but performs worse as the GSNR increases. This is expected due to the vanishing signal strength assumption at the LOB detector. The sign correlator $(\mathrm{SC})$, however, gives significantly better performance than the Gaussian detector, but is nonetheless noticeably worse than the optimal detector.

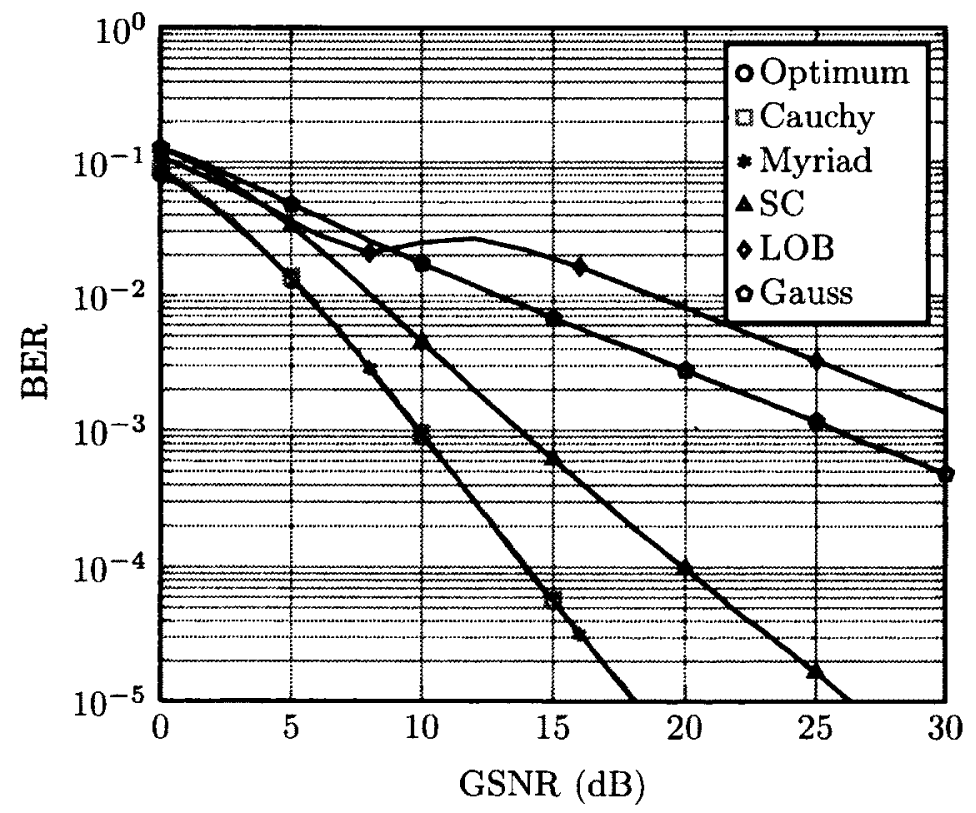

Figure 3.14: Performance comparison of suboptimal detectors with $\alpha=1.5, N=4$. 
Similar results hold when $\alpha$ is increased to $\alpha=1.9$, as shown in Figure 3.15, although some differences between the performance of the optimal, Cauchy, and myriad detectors are apparent. In particular, the Cauchy detector is clearly suboptimal at low GSNR, while the same is true for the myriad filter at high GSNR.

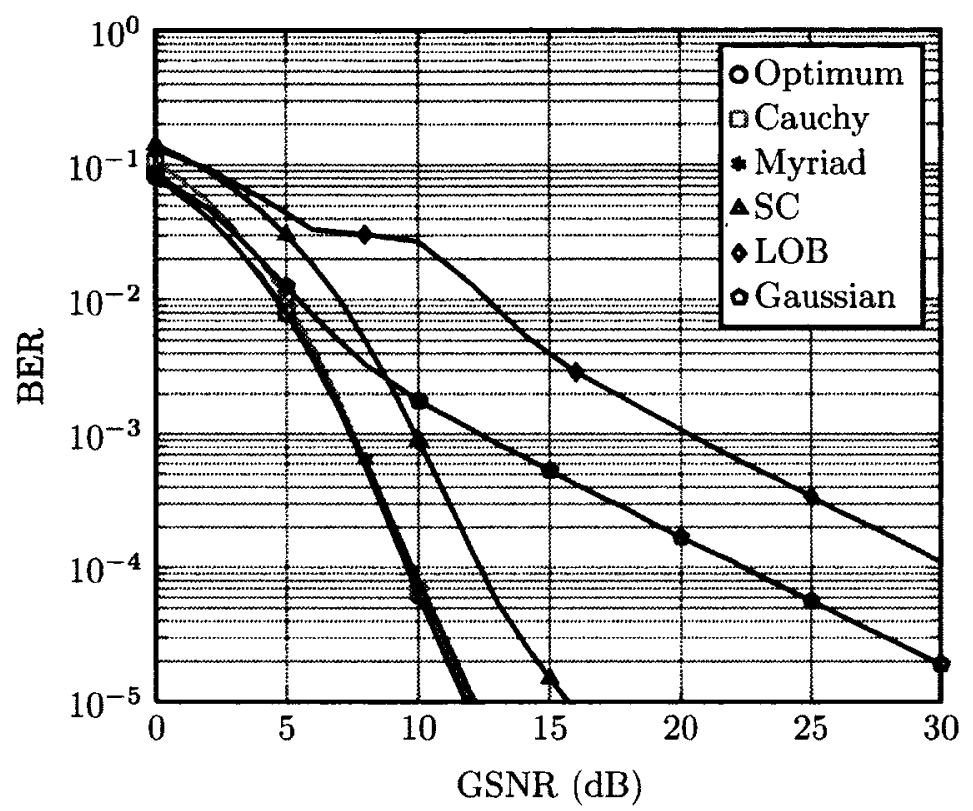

Figure 3.15: Performance comparison of suboptimal detectors with $\alpha=1.9, N=4$.

Figure 3.16 shows the performance of the SL detector compared to the Gaussian and optimal detectors. To compare the performance of the SL detector with different values of the threshold, we fix $A=1$ and vary $\gamma$ according to the GSNR. It can be seen that the soft limiter (SL) detector, with the arbitrary fixed threshold value of $\eta=30$ as proposed in [50], gives almost no performance improvement over the Gaussian detector. When the SL detector is used with smaller values, it gives different performance gains compared to the Gaussian detector. Although we believe that it is meaningless to choose these or any other fixed values, up to the best of our knowledge, no clear way to set the threshold has been proposed in the literature. We have observed that, at any given value of the threshold, the SL detector shows better 
performance at a certain value of GSNR, $A$ and/or $\gamma$. So, the threshold value can be set along with other system parameters to give better or worse performance. As a result, there is no fixed value of the threshold that would give a fair performance comparison. We therefore prefer to use the value that has been already proposed in the literature. In this thesis, we will show how to choose a more accurate threshold value.

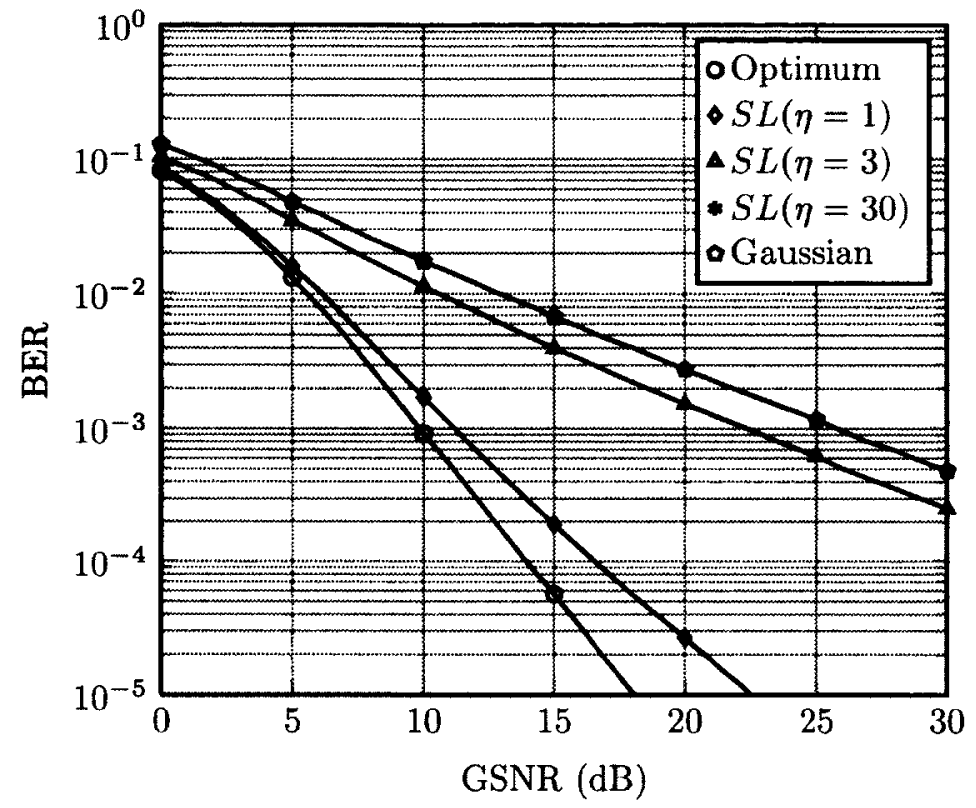

Figure 3.16: Performance of SL detector with different threshold values with $\alpha=$ $1.5, N=4$.

In general, for all values of $N$ and for all $\alpha<2$, the Gaussian detector performs much worse than the optimal detector. The LOB detector also performs poorly, except at very low GSNR when $\alpha<1.5$. The sign correlator is much better, except when $N=2$. Its performance improves as $N$ increases, and is better when $N$ is odd than even. This is because there is no possibility of any ambiguity in the majority decision rule when $N$ is odd. The Cauchy detector and myriad filter both perform well, with the myriad filter having the advantage at low GSNR while the Cauchy detector is better at high GSNR. Except as noted above, there is very little in the published 
literature to explain the differences in the performance of these suboptimal detectors, and one of the objectives of this thesis is to provide a framework for analyzing these detectors to provide appropriate explanations, as will be presented in Chapter 4 .

\subsubsection{Summary}

The optimal ML detector for signals in $\mathrm{S} \alpha \mathrm{S}$ noise is impractical due to its complexity. There are feasible simple suboptimal detectors, however, there is no methodology for designing and improving these suboptimal detectors. For example, it was found by observation that the Cauchy detector has robust performance for different values of $\alpha$, which is better than many suboptimal detectors; however, there is no justification for its robustness. When simple detectors are needed, the Gaussian (linear) detector was chosen with a pre-processing device to shape the received signal by eliminating the impulses in the received samples to improve the Gaussian detector performance. However, the pre-processing device was designed based on the locally optimal nonlinearity which is based on a limited and inaccurate criterion. Moreover, instead of using design criteria that consider the underlying noise statistics, this way of design forces the received signal to have a Gaussian-like distribution just to use the Gaussian detector, which is not necessarily a good way of design.

In the next chapter, a new methodology for suboptimal detector design is proposed. This methodology depends on understanding the behavior of the optimal detector by analyzing its decision regions. The proposed analysis gives insight on the optimal processing of the received samples and the effect of the noise impulses on the optimal decisions. The proposed methodology succeeds in justifying the performance of several suboptimal detectors and it also gives a way to improve the existent suboptimal detectors and develop new low-cost detectors as well. 


\section{Chapter 4}

\section{Optimal Decision Regions: A Framework to Design Suboptimal Detectors for Signals in SoS Noise}

\subsection{Introduction}

In this chapter, we present the main contribution of the thesis on the detection of signals in $\mathrm{S} \alpha \mathrm{S}$ noise. Firstly, we propose to use the optimal decision regions to study the behavior of the optimal detector which gives insight into the effect of the noise impulses on the optimal decisions. Based on this analysis, accurate justification for the performance of several suboptimal detectors is proposed. Secondly, an adaptive suboptimal detector is introduced as a direct application of using the proposed analysis to improve the soft limiter detector. Finally, a more generic simple suboptimal detector is proposed based on a linear piecewise approximation of the log-likelihood ratio of the received samples which gives robust near-optimal performance at low complexity. 


\subsection{Optimal Decision Regions}

In this section, we propose to analyze the decision regions of the optimal detector to understand the effect of the noise impulses on the optimal decisions. The received samples are modeled as

$$
r_{k}=s+n_{k} \quad k=1, \ldots, N
$$

where the transmitted symbol is $s \in\{ \pm A\}$ and $\left\{n_{k}\right\}$ are $N$ independent S $\alpha S$ noise samples per symbol.

When $N=1$, it can be shown that the optimal ML detector reduces to the Gaussian detector. However, when $N>1$, the ML detector behaves differently while the Gaussian detector has poor performance as shown in Chapter 3. The behavior of the optimal detector is analyzed when $N=2$, which gives tractable analysis and explains the effect of the noise impulses on the optimal decisions. In the proposed analysis, the optimal decision boundaries are calculated which will be used to justify and improve the performance of many suboptimal detectors.

To calculate the decision regions, the optimal decision boundaries are calculated by equating the conditional joint $p d f \mathrm{~s}$ as follows:

$$
f_{\alpha}\left(r_{1}, r_{2} \mid s=-A\right)=f_{\alpha}\left(r_{1}, r_{2} \mid s=+A\right)
$$

For the case of the Cauchy distribution $(\alpha=1)$, the decision boundaries can be obtained analytically given the Cauchy $p d f[8]$ :

$$
f\left(r_{k} \mid s\right)=\frac{\gamma}{\pi} \frac{1}{\gamma^{2}+\left(r_{k}-s\right)^{2}}
$$




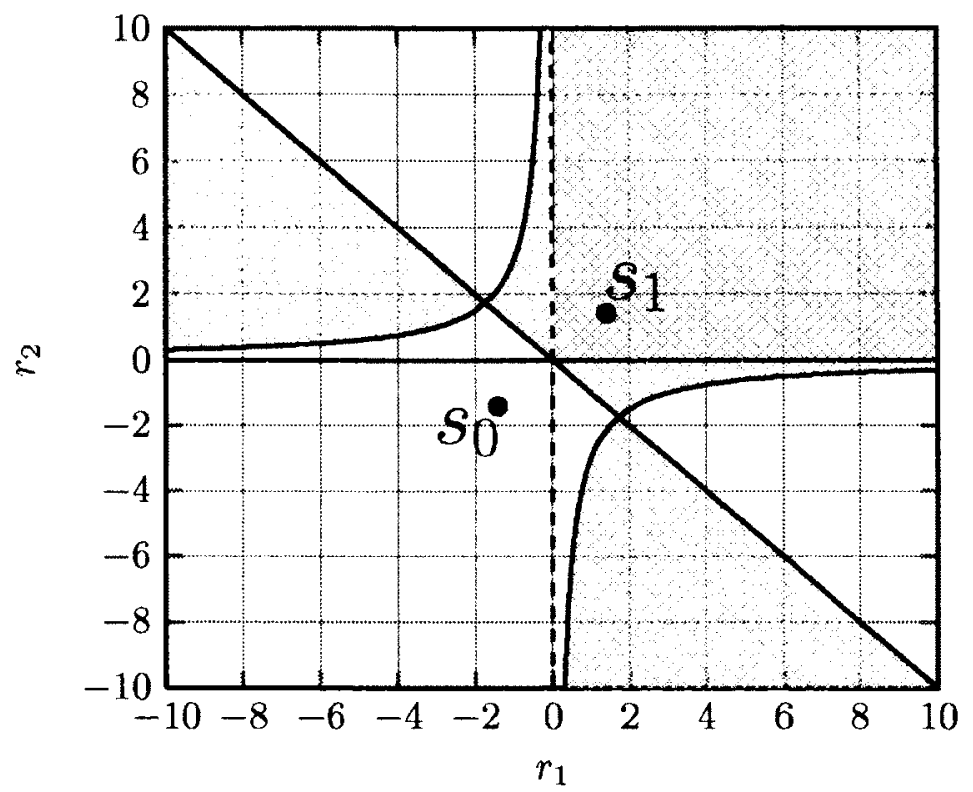

Figure 4.1: Optimal decision regions with $\alpha=1$ and $\gamma=1$. Shaded area: decide $s_{1}$, white area: decide $s_{0}$.

By substituting (4.3) into (4.2), the decision boundaries can be solved as

$$
\begin{aligned}
& r_{2}=-r_{1} \\
& r_{2}=-\frac{\gamma^{2}+A^{2}}{r_{1}}
\end{aligned}
$$

The optimal decision regions are illustrated in Figure 4.1 for $\alpha=1$. As shown in the figure, the $\mathrm{S} \alpha \mathrm{S}$ optimal detector has disjoint decision regions which result from the heavy tail effect of the noise distribution. The reason behind those disjoint decision regions can be explained by showing the joint probability density function of the received samples, $f_{\alpha}\left(r_{1}, r_{2}\right)$. Figures 4.2 and 4.3 show the joint conditional probability density function for Cauchy and Gaussian distributions, $\alpha=1$ and 2, respectively. Also, in Figures 4.4 and 4.5, both Cauchy and Gaussian joint conditional probability density functions are shown on log scale to better illustrate the heavy tail effect on the decision region. 


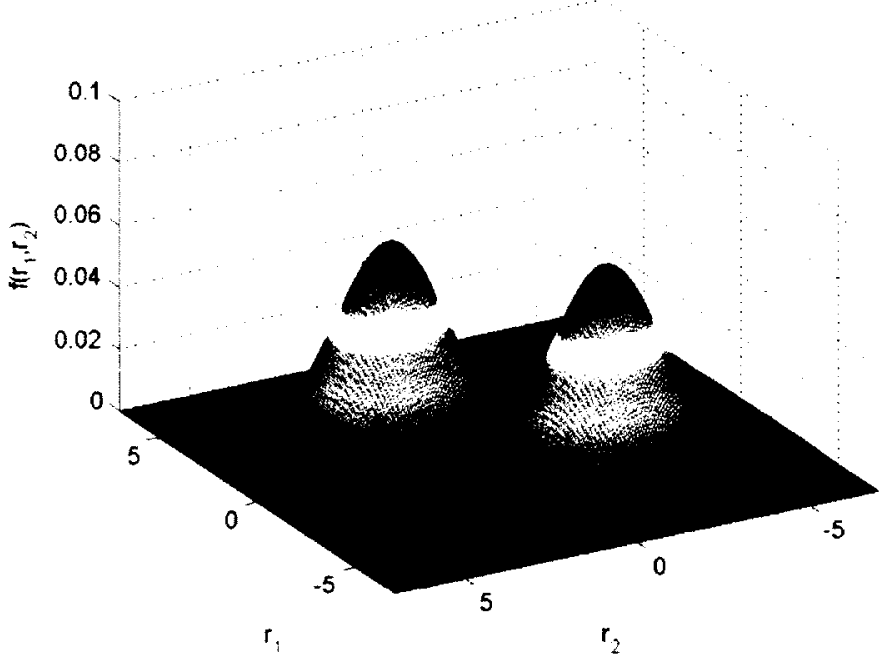

Figure 4.2: Gaussian joint conditional probability density functions.

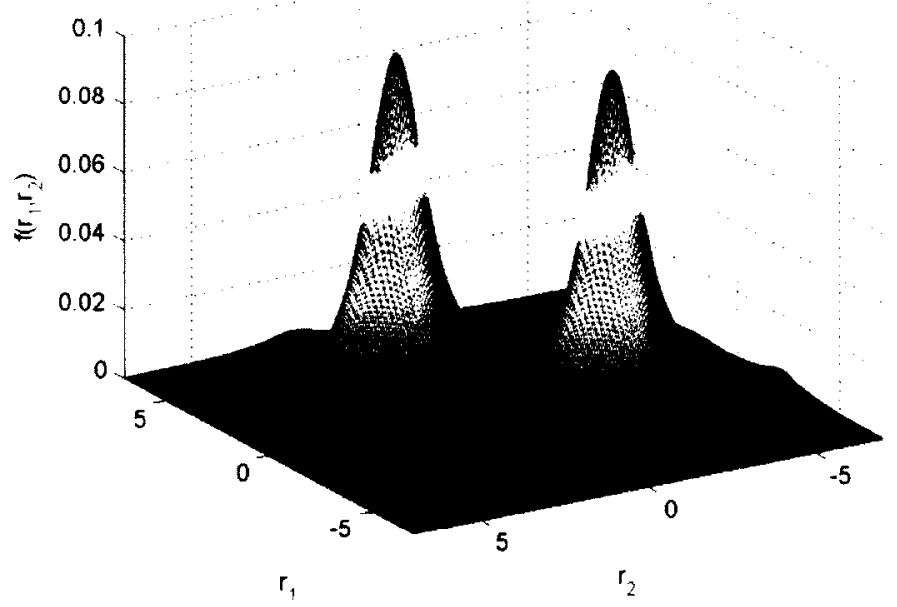

Figure 4.3: Cauchy joint conditional probability density functions. 


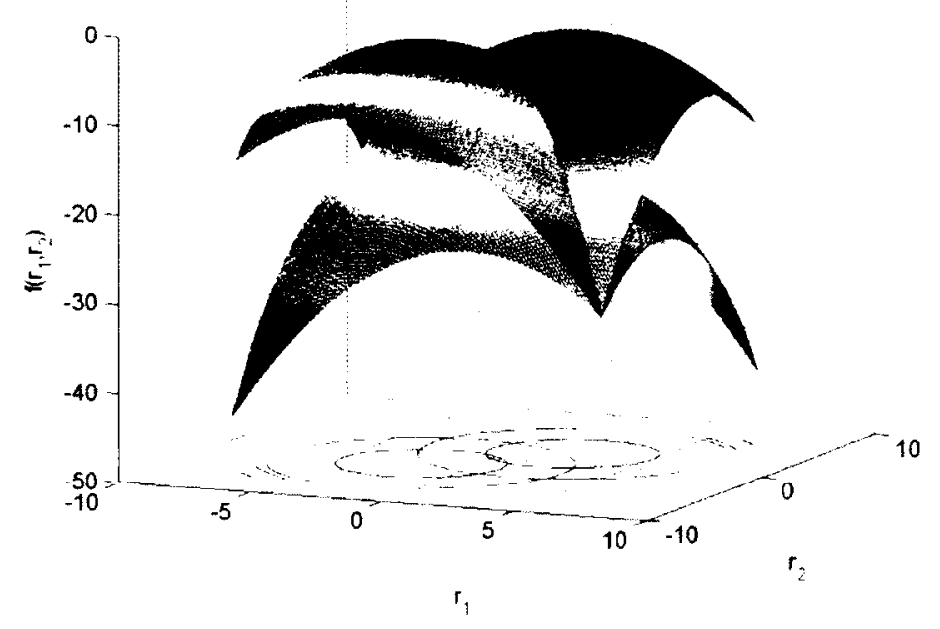

Figure 4.4: Gaussian joint conditional probability density functions on log scale.

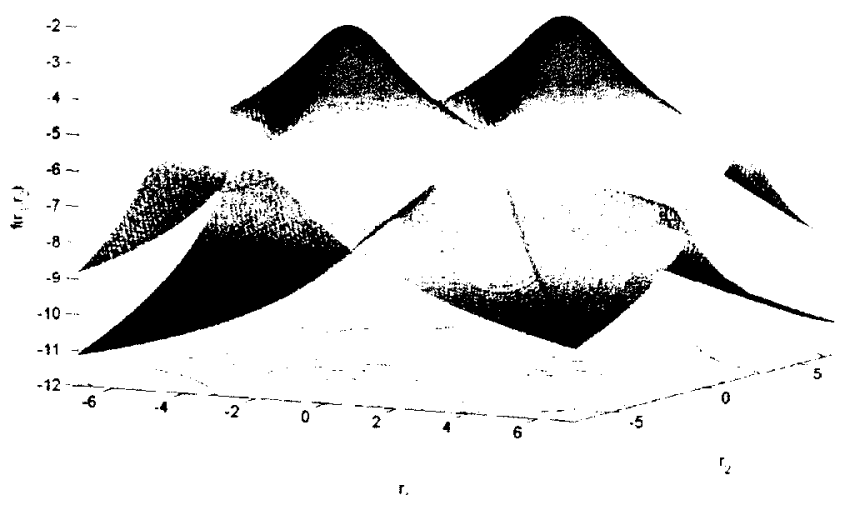

Figure 4.5: Cauchy joint conditional probability density functions on log scale.

It can be observed that if the noise in one of the received samples is an impulse, it is most likely that it will be detected correctly, even if the impulse has a sign opposite to that of the transmitted symbol. For different values of $\alpha$, Figure 4.6 shows the optimal decision boundaries (found numerically) in the lower part of the fourth quadrant only, evaluated at different values of GSNR ${ }^{1}$. As shown in the figure,

\footnotetext{
${ }^{1}$ In this figure $\gamma$ is fixed at 1 and $A$ is adjusted according to the GSNR.
} 


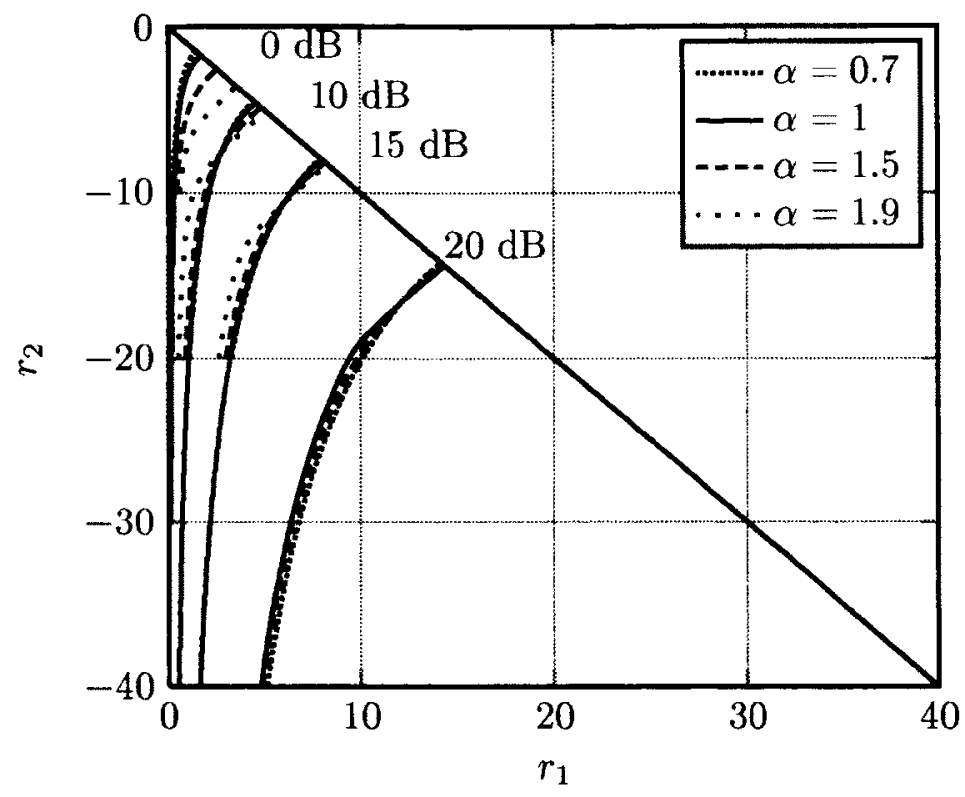

Figure 4.6: Optimal curved decision boundaries.

the optimal decision boundaries for $\alpha \neq 1$ shares the linear boundary in (4.4a) and the curved boundaries become close to the case when $\alpha=1$ as the GSNR increases. So, at higher GSNR, the curved boundaries for different values of $\alpha$ can be approximated by $(4.4 b)$.

\subsection{Performance Justification of the Existing De- tectors}

By comparing the decision regions for the suboptimal detectors with those of the optimal detector, we gain considerable insight into the behavior of the suboptimal detectors when processing the received samples. This helps justify the performance of several suboptimal detectors and provides a way to improve them. 


\subsubsection{Gaussian Detector}

This detector has one linear decision boundary, as given by (4.4a). As shown in Fig. 4.1, there are wide decision regions where the Gaussian detector makes different decisions from the optimal one. The difference in the decision results from the difference between the heavy-tail and Gaussian noise models. Furthermore, when the noise has a heavy tail, there is a high probability that the received samples will fall in these erroneous decision regions. Therefore, the Gaussian detector's performance is expected to be much worse than the optimal one, even for values of $\alpha$ near 2 .

\subsubsection{Soft Limiter Detector}

This detector clips the received samples to a certain threshold then it applies the Gaussian detector. The motivation behind the clipping of the received signal is mentioned in [50] as the signal to noise ratio will be improved and therefore the detectors performance will be improved as well. However, based on the proposed analysis in this section, we provide a better explanation for the benefit of the soft limiter in terms of the resultant decision region. A more detailed discussion is introduced in section 4.4 to justify and improve the performance of the SL detector.

\subsubsection{Cauchy Detector}

By observing the optimal decision boundaries in Fig. 4.6, it can be seen that the optimal boundaries for all values of $\alpha$ have the same linear boundary and different, but similar, curved boundaries. Therefore, the decision boundary for the Cauchy detector can be considered as a better fit to the optimal boundaries (for any value of $\alpha$ ) than the other suboptimal detectors. Therefore, this observation justifies the robust performance of the Cauchy detector for different values of $\alpha$ and the advantage it has over many suboptimal detectors. 


\subsubsection{Myriad Filter}

The decision boundaries of the myriad filter, for the case when $\alpha=1$, are:

$$
\begin{aligned}
& r_{2}=-r_{1} \\
& r_{2}=-\frac{\kappa^{2}+A^{2}}{r_{1}}
\end{aligned}
$$

where $\kappa=\gamma^{\frac{1}{\alpha}} \sqrt{\frac{\alpha}{2-\alpha}}$. Note that $\kappa=\gamma$ when $\alpha=1$. So, it can be shown that, the myriad filter tries to fit its decision boundaries to the optimal boundaries by using the Cauchy boundaries with variable noise dispersion $\kappa$. Therefore, the performance of the myriad filter is almost equal to that of the Cauchy detector when $\alpha$ approaches one and it is slightly better than the Cauchy detector for higher values of $\alpha$ where $\kappa$ succeeds in fitting the optimum decision boundaries more closely. It is worth noting that the performance of the myriad filter is limited by the accuracy of the shape of the Cauchy detector boundaries which form the curve fitting process. Also, there is no optimized way to calculate $\kappa$. By using the proposed analysis, a better way to optimize $\kappa$ to closely fit the optimum boundaries for different values of $\alpha$ can be suggested.

\subsubsection{Locally Optimum Baysian Detector}

The decision boundaries of this detector, for the case when $\alpha=1$, are:

$$
\begin{aligned}
& r_{2}=-r_{1} \\
& r_{2}=-\frac{\gamma^{2}}{r_{1}}
\end{aligned}
$$

It can be shown that the boundaries of the locally optimal Baysian detector approximate the optimal decision boundaries for the case when $\alpha=1$ for low GSNR values. 


\subsubsection{Sign Correlator Detector}

The decision boundaries of this detector is straight forward as it depends on the majority rule, see (3.13),

$$
r_{i}=0 \quad i=1,2, \cdots N,
$$

which results in $2^{N}$ equal decision regions. For simplicity, when $N=2$, the sign correlator detector has the same decision as the optimal one in the first and third quadrant. However, in the second and the fourth quadrant, where $\operatorname{sgn}\left(r_{1}\right)+\operatorname{sgn}\left(r_{2}\right)=$ 0 , it flips a coin, i.e., $P_{e}=0.5$, which explains the poor performance of the sign correlator detector when $N=2$. In general, when $N$ is even, the sign detector flips a coin in the regions where $\sum_{i} \operatorname{sgn}\left(r_{i}\right)=0$ which explains the worse performance of the sign correlator detector relative to the optimal one for the case when $N$ is even compared to the case when $N$ is odd.

\subsection{Proposed Simplified Near-Optimal Detectors}

Based on the optimal decision regions, two simplified suboptimal detectors are proposed to give near-optimal performance with low complexity. The proposed detectors are designed in a way to have decision regions with closer fit to the optimal decision regions while maintaining low-complexity. First, we improve the performance of the soft limiter detector by using an adaptive threshold which tunes the decision regions in order to closely approximate the optimal decision regions. Another suboptimal detector, the piecewise linear detector, has been proposed to have decision regions with better fit to the optimal ones. In this section, we present those detectors and the limitations of the proposed design. 


\subsubsection{Soft Limiter Detector with an Adaptive Threshold}

Due to its simplicity, the soft limiter detector is appealing in practical implementations. It simply uses a clipping device at the input of the Gaussian detector, so it adds almost no complexity to the conventional Gaussian detector. However, its performance is far from the optimal. The threshold value is the main parameter that affects the performance of the soft limiter detector. For a given GSNR, $A$ and/or $\gamma$, setting the threshold too high results in what is essentially a Gaussian detector, whereas, setting the threshold too low results in what is essentially a sign correlator. In [50], arbitrary fixed threshold values were used. In [31] and [48], it was argued that the optimal threshold value should minimize the probability of error. However, no clear method to calculate the threshold was provided, and the probability of error was calculated assuming that a locally optimal nonlinearity was applied to the received samples, even though the limiter does not even crudely approximate the locally optimal nonlinearity. In this section, we investigate the soft limiter detector behavior in more detail. This investigation shows the effect of the threshold value on the detector performance. Then, an adaptive threshold is proposed to improve the detector's performance. Unlike the fixed threshold, the proposed threshold depends on the signal level.

\subsubsection{Soft Limiter Detector Analysis}

Due to symmetry, only the fourth quadrant in the decision region plane will be analyzed. In this quadrant, the decision regions for the soft limiter and Gaussian detectors are illustrated in Fig. 4.7 where the soft limiter detector uses a threshold value $\eta$. It can be seen that the limiter and Gaussian detectors give the same decision when the received sample falls in region $R_{1}$ and $R_{2}$; they differ only in regions $R_{3}$ and $R_{4}$. The soft limiter clips all points in these regions to $(\eta,-\eta)$, which is on the linear boundary. 


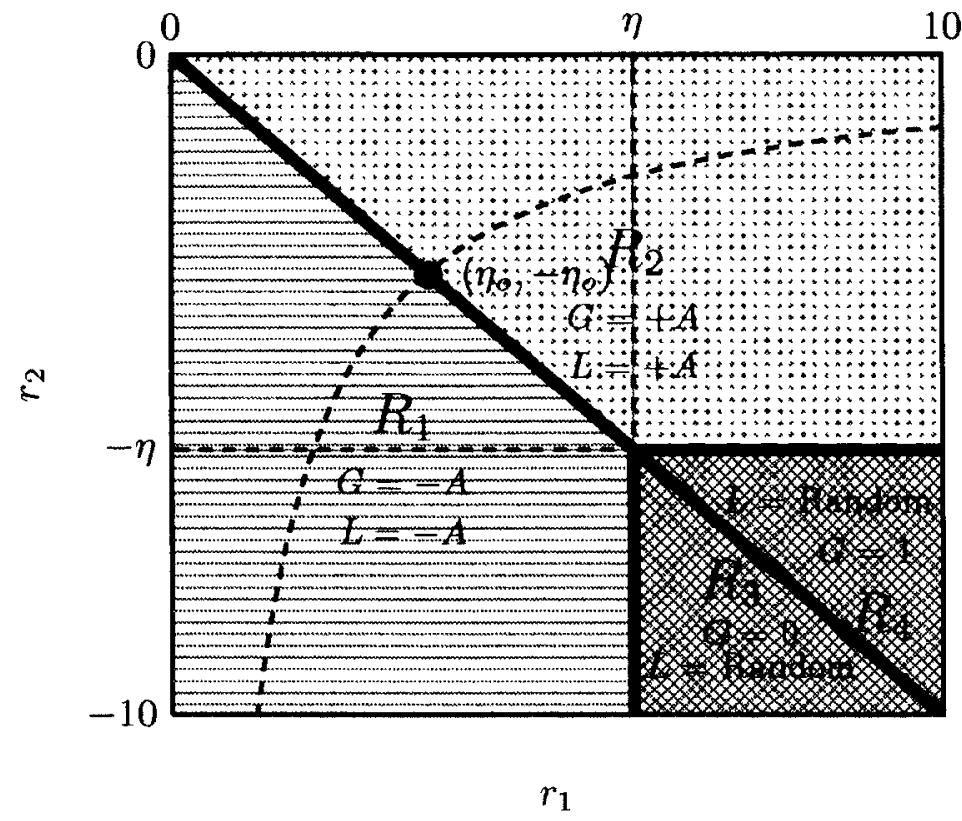

Figure 4.7: Comparison between the decision regions of the soft limiter (L), Gaussian detectors $(G)$.

By assuming any point on the boundary is selected randomly by means of a coin toss, samples that fall in these regions will be detected as $s=+A$ half the time and as $s=-A$ half the time, so the probability of error for the limiter detector will be 0.5 when the sample falls in these regions. On the other hand, the Gaussian detector will always choose $s=-A$ for region $R_{3}$ and $s=+A$ for region $R_{4}$. But, according to Figure 4.1, the optimal detector decides in favour of $s=+A$ when the received sample falls in region $R_{3}$, which means $P_{r}\left\{s=+A \mid r_{1}, r_{2}\right\}>P_{r}\left\{s=-A \mid r_{1}, r_{2}\right\}$ in this region, so $P_{r}\left\{s=+A \mid r_{1}, r_{2}\right\}>0.5$. Therefore the probability of error for the Gaussian detector is greater than 0.5 when the sample falls in $R_{3}$ (and, by similar reasoning, in $R_{4}$ ). As a result, the soft limiter detector is less likely than the Gaussian detector to make an error if the received samples fall within $R_{3}$. This detector behavior explains the performance improvement that the soft limiter detector has over the Gaussian detector. 


\subsubsection{Adaptive Soft Limiter (ASL) Detector}

To improve the performance of the soft limiter detector, an approximate value of the threshold may be chosen such that the regions $R_{3}$ and $R_{4}$ are as large as possible without introducing errors in the other decision regions. By inspecting the decision regions in Fig. 4.7, it can be shown that setting the threshold value at the intersection of the decision boundaries, $\eta_{o}$, achieves the largest decision region, $R_{3}$, without affecting the other decision regions. This threshold can be calculated analytically when $\alpha=1$ and $N=2$ to be $\eta_{o}=\sqrt{\gamma^{2}+A^{2}}$ which assumes that the noise dispersion, $\gamma$, is known to the receiver.

Because of the complex analysis of the decision boundaries for $N>2$, we used simulation BER results for different values of $\eta$ to calculate the optimal value of the threshold. The results show that the optimal threshold is upper bounded by $\eta_{o}$. Also, this threshold can be further simplified for large GSNR values to be $\eta_{o}=A$. The simulation results show that this threshold can be used as a good approximation for the case when $\alpha \neq 1$ with different values of $N$.

\subsubsection{Simulation Results and discussion}

The performance, in terms of the probability of error, of the soft limiter detector that uses the proposed adaptive threshold (ASL) is evaluated by simulation. Based on the proposed analysis, the simulation results compare the performance of the Gaussian, SL detector with fixed threshold, adaptive soft limiter, and optimal detectors. The fixed SL detector uses a fixed threshold of $\eta=30$ as used in [50], whereas the adaptive soft limiter uses $\eta=\eta_{o}$.

Figure 4.8 shows the performance when $\alpha=1.5$ and $N=2$. It can be seen that the performance of the fixed SL and the Gaussian detector are almost the same. This performance is expected because the threshold value that controls the decision 
regions $R_{3}$ and $R_{4}$ in Figure 4.7, is much higher than $A$. So, these regions are located far from the center of the joint distribution of the received samples, which decreases the advantage that the SL detector has over the Gaussian detector. As can be seen, the ASL detector gives a slight gain over the fixed limiter, although its performance is still far from optimal, because region $R_{3}$ is large.

The advantage of the adaptive soft limiter is more pronounced when $N$ is larger, as shown in Figure 4.9 and 4.10 for $N=10$ and $\alpha=1.5$ and 1.9. The ASL detector provides performance that is nearly identical to that of the optimal detector, whereas the Gaussian and fixed threshold SL detectors are very ineffective. Although the SC gives better performance than the Gaussian one, it is still behind the optimal by about $2 \mathrm{~dB}$. When $N$ is odd, the SC detector is close to the proposed ASL detector. Even the Cauchy detector, whose complexity becomes impractical as $N$ increases, suffers from degraded performance for large $\alpha$. This indicates that the soft limiter detector with the proposed adaptive threshold can achieve very good performance, even approaching the optimal detector in some cases, at almost no additional complexity over the conventional Gaussian detector. 


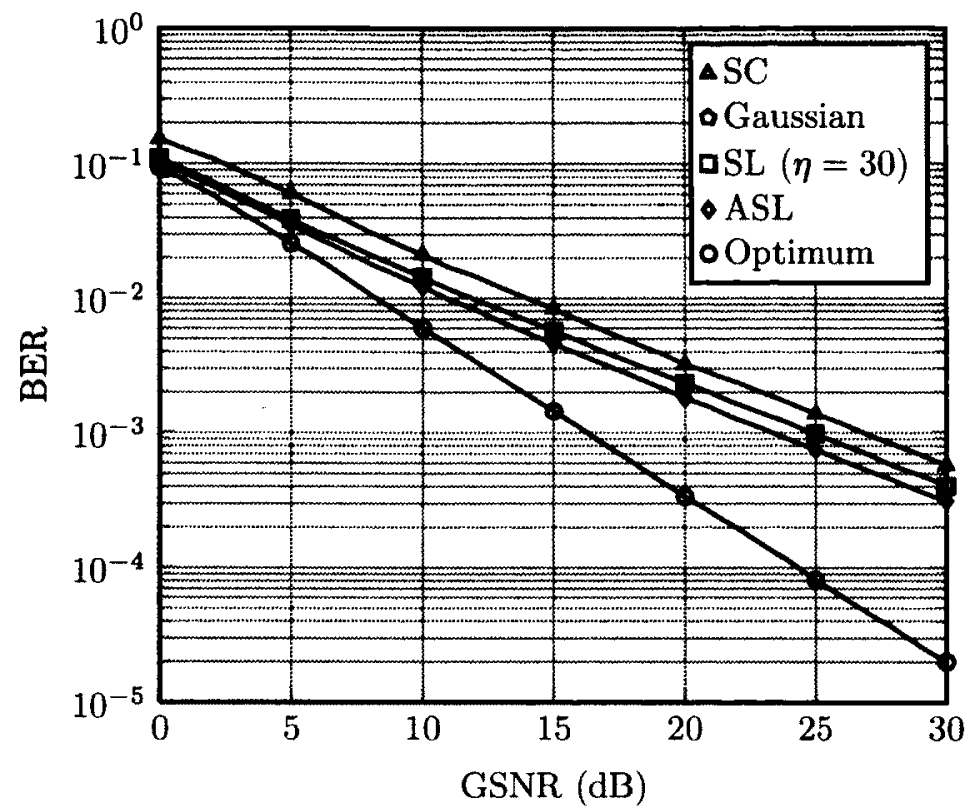

Figure 4.8: Performance comparison of the ASL detector with $\alpha=1.5, N=2$.

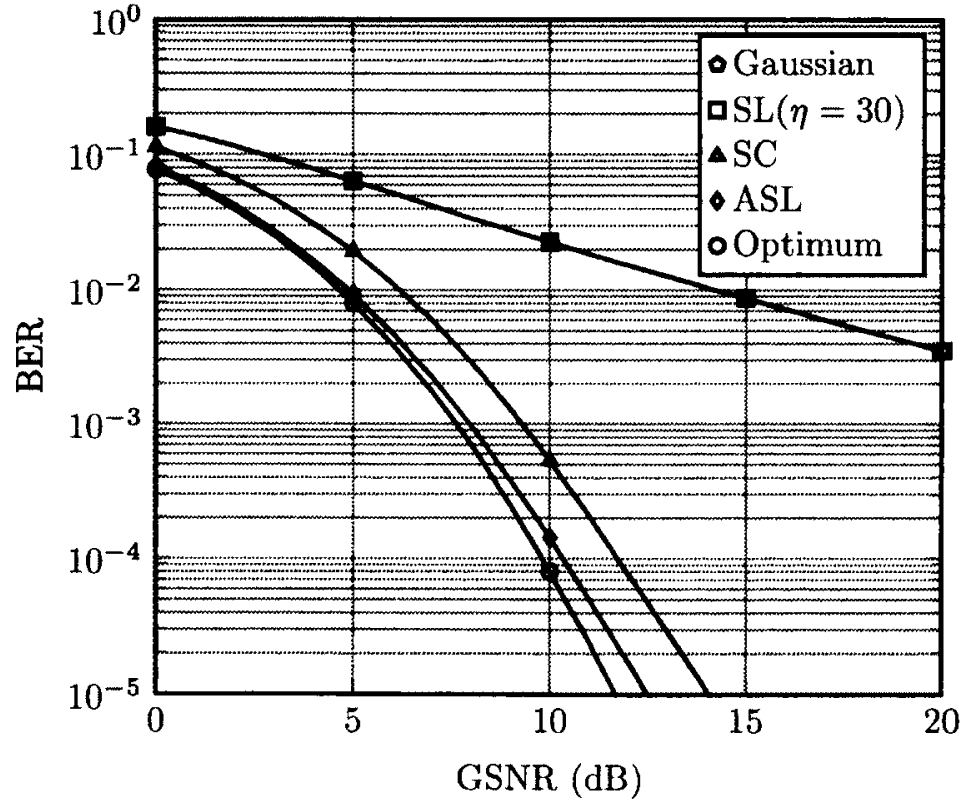

Figure 4.9: Performance comparison of the ASL detector with $\alpha=1.5, N=10$. 


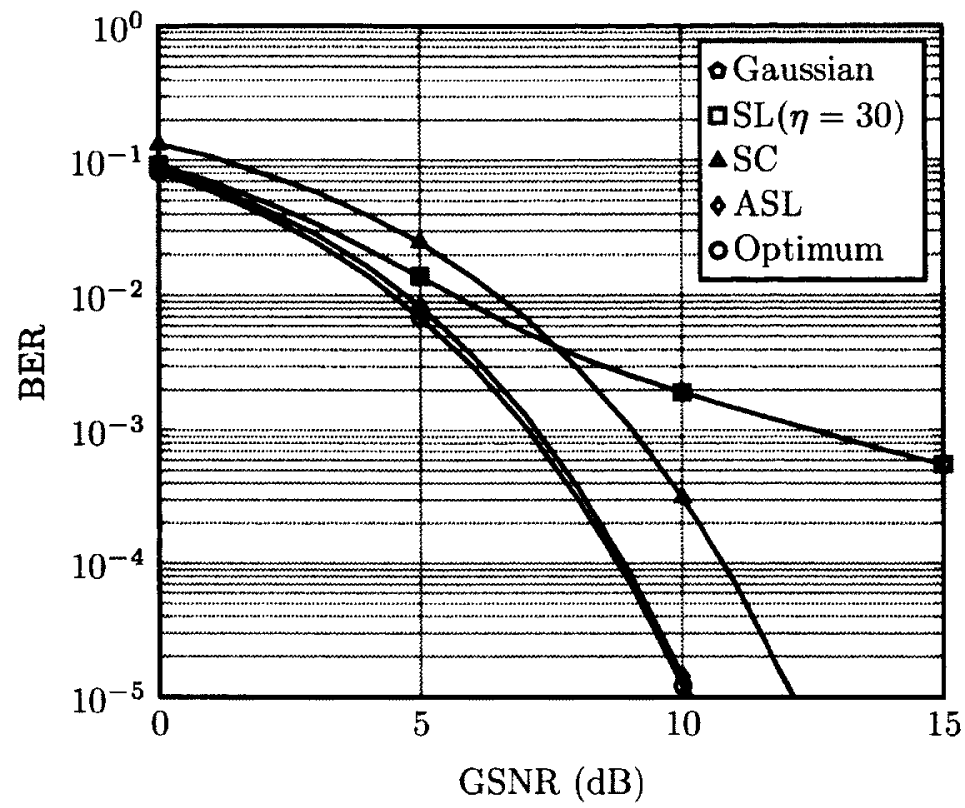

Figure 4.10: Performance comparison of the ASL detector with $\alpha=1.9, N=10$.

To help explain why the performance of the adaptive soft limiter improves so much as $N$ increases, it is instructive to imagine the ambiguous decision regions where the adaptive limiter randomly makes its decision (that is, consider the region equivalent to $R_{3}$ and $R_{4}$, but in $N$-space). These regions only occur when $N$ is even. As $N$ increases, these regions cover a decreasing fraction of the signal space. Assuming for the moment that the threshold is $\eta=0$ (as for the sign correlator), then the ambiguous region covers $\frac{1}{2}$ of the signal space when $N=2$ but only $\frac{6}{16}$ of the space when $N=4$, and $\left(\begin{array}{c}N \\ \frac{N}{2}\end{array}\right) / 2^{N}$ in general. Furthermore, a larger number of large impulses are needed for the received sample to fall in these regions as $N$ increases, so the likelihood of this occuring decreases. It should be noted that this only partially explains the reduction in the gap between the adaptive limiter and the optimal detector as $N$ increases. When $N$ is odd, small gains are also evident as $N$ increases, but the reasons are less clear. It is likely not so much that the decision regions of the adaptive limiter more closely match those of the optimal detector as $N$ increases but rather that the 
received samples are less likely to fall in areas where the regions differ.

\subsubsection{Low-Complexity Piecewise Detector}

In this section, we consider the design of a suboptimal detector for the case where only two independent samples are available at the receiver $(N=2)$. This case is practically appealing when it is justified by the use of two antennas at the receiver. Although the simple adaptive soft limiter detector proposed in the previous section has almost $2 \mathrm{~dB}$ gain over the Gaussian detector, it is still far from the optimal performance as can be seen in Figure 4.8. Also, although the Cauchy detector has near-optimal performance, it is more complex. Based on the framework proposed in this chapter, we propose a low complexity piecewise detector that approximates the decision boundaries of the optimal detector using linear segments. The performance of the proposed piecewise detector approaches the optimal one for different values of $\alpha$ with much less complexity compared to the optimal ML detector. Also, by using the proposed framework, the performance of the optimal and the proposed detectors can be evaluated analytically which was not attainable in the past. Based on the analysis of the optimal decision regions, we propose a new suboptimal detector that linearly approximate the optimal decision boundaries shown in Figure 4.1. In this detector, the curved boundaries in $(4.4 \mathrm{~b})$ are approximated by using piecewise symmetric linear segments passing through the boundaries cross point $\left( \pm a_{o}, \mp a_{o}\right)$ as shown in Figure 4.11. Due to symmetry, the fourth quadrant is considered where, for $0 \leq r_{1} \leq a_{o}$ the linear segment is

$$
r_{2}=m r_{1}-(m+1) a_{o}
$$

where $a_{o}=\sqrt{\gamma^{2}+A^{2}}$ and $m$ is the slope of the segment. The linear segment crosses the boundary (4.4b) in two points $\left(a_{0},-a_{o}\right)$ and $\left(\frac{a_{o}}{m},-a_{o} m\right)$. When $m=1$, the line in 


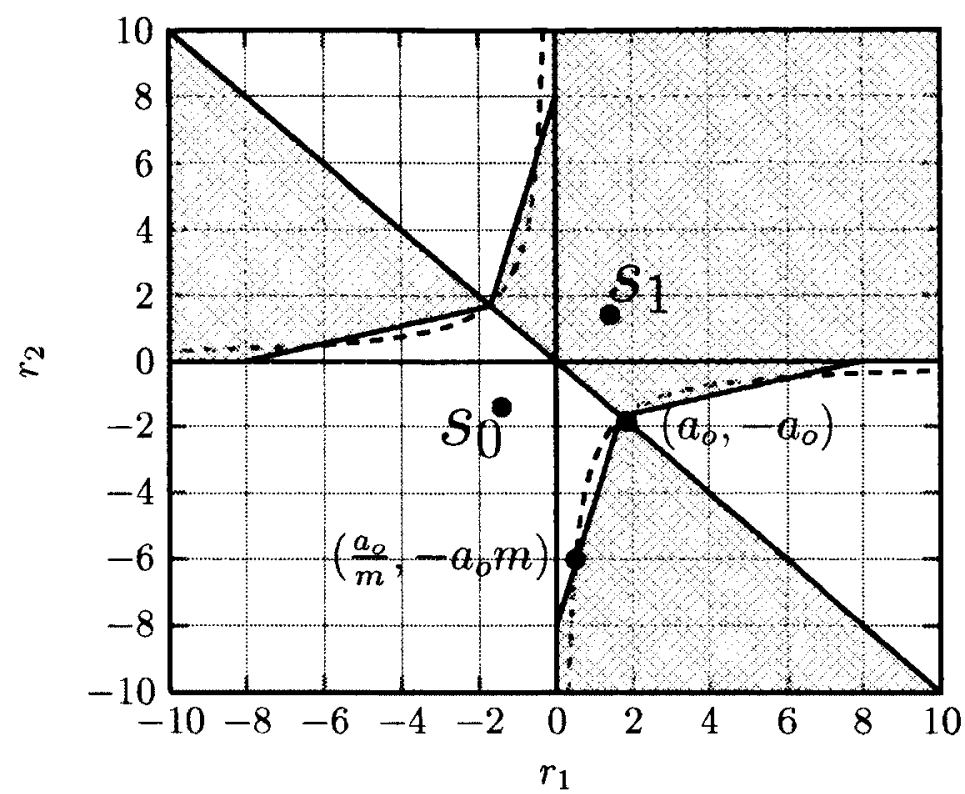

Figure 4.11: Decision boundaries of the piecewise detector.

(4.8) is a tangent to the boundary at the point $\left(a_{o},-a_{o}\right)$, while at $m>1$ and $m<1$, the line forms the two segments shown in Figure 4.11. As can be seen, the accuracy of the linear approximation in (4.8) depends on the slope $m$. We propose to choose $m$ such that the excess probability of error, $\beta$, is minimized. The excess probability of error is defined as the difference between the probability of bit error of the piecewise and optimal detectors. By comparing the decision regions in which both the optimal and the piecewise detector have different decisions, the excess probability of error, $\beta$, is reduced to:

$$
\beta=2 \times\left[\iint_{R_{1}} \Lambda_{\alpha}\left(r_{1}, r_{2}\right) d r_{2} d r_{1}+\iint_{R_{2}} \Lambda_{\alpha}\left(r_{1}, r_{2}\right) d r_{2} d r_{1}\right]
$$

where

$$
\Lambda_{\alpha}\left(r_{1}, r_{2}\right)=f_{\alpha}\left(r_{1}, r_{2} \mid s=+A\right)-f_{\alpha}\left(r_{1}, r_{2} \mid s=-A\right)
$$

and $R_{1}:\left\{r_{1}=0 \rightarrow \frac{a_{o}}{m}, r_{2}=-\frac{a_{o}^{2}}{r_{1}} \rightarrow-r_{1}\right\}$ and $R_{2}:\left\{r_{1}=\frac{a_{o}}{m} \rightarrow a_{o}, r_{2}=-r_{1} \rightarrow-\frac{a_{o}^{2}}{r_{1}}\right\}$. 


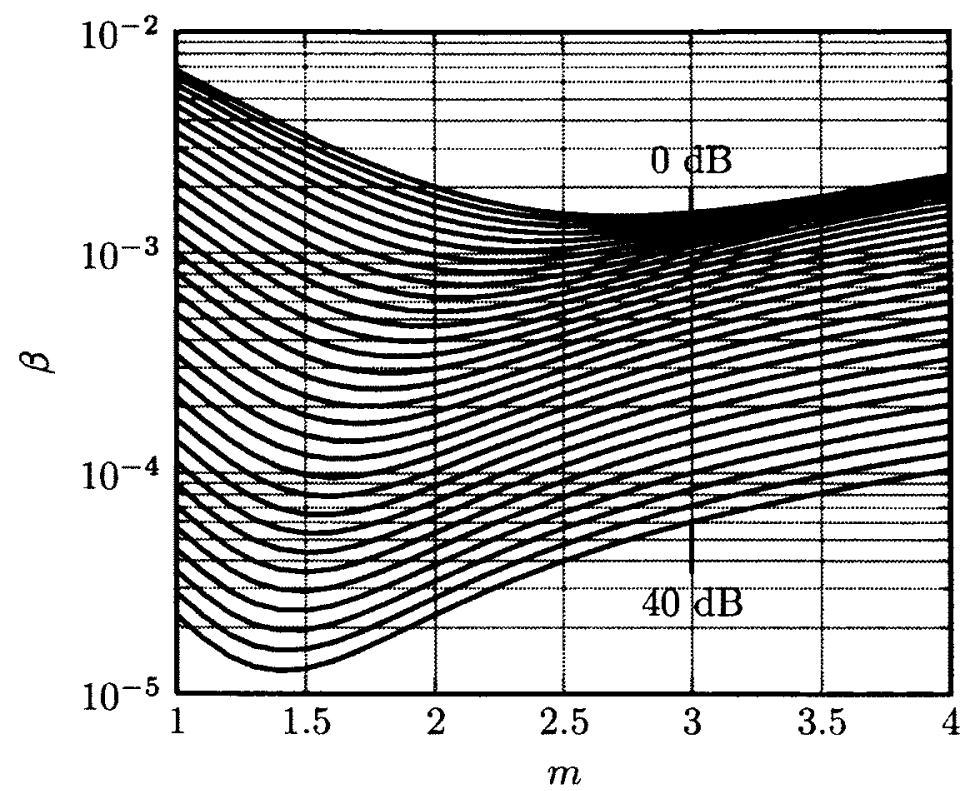

Figure 4.12: The excess probability of error $(\beta)$ evaluated at GSNR $=0,1, \ldots, 40$ dB with $\alpha=1$.

It is difficult to minimize $\beta$ analytically, so instead it is evaluated numerically. Figure 4.12 shows $\beta$ at different values of the GSNR. The optimal slope, $m_{\text {opt }}$, can be calculated numerically for different values of $\gamma$ and GSNR and stored in memory. It would also be fitted linearly with GSNR. However, Figure 4.12 shows that the difference between the excess probability of error, $\beta$, when $m=1$ and $m=m_{\text {opt }}$ is small enough to use $m=1$ as a simple good approximation of the optimal slope, bearing in mind that the excess probability of error is usually small compared to the total probability of error.

\subsubsection{Performance Evaluation and Simulation Results}

In this section, the performance of the optimal, the Gaussian and the piecewise detectors will be evaluated analytically in the case when $\alpha=1$.

\section{Optimum Detector}

By using the decision regions in Figure 4.1, the conditional probability of correct 
decision can be evaluated as follows:

$$
\begin{aligned}
& P_{c \mid-A}= \iint_{Z} f_{\alpha}\left(r_{1}, r_{2} \mid s=-A\right) d r_{2} d r_{1} \\
&= 2 \times \int_{r_{1}=0=-\frac{a_{0}^{2}}{r_{1}}}^{a_{o}} \int_{\alpha}^{-r_{1}}\left(r_{1}, r_{2} \mid s=-A\right) d r_{2} d r_{1}+ \\
& 2 \times \int_{r_{1}=a_{o}}^{\infty} \int_{r_{2}=-r_{1}}^{-\frac{a_{o}^{2}}{r_{1}}} f_{\alpha}\left(r_{1}, r_{2} \mid s=-A\right) d r_{2} d r_{1}+ \\
& \int_{r_{1}=-\infty}^{0} \int_{r_{2}=-\infty}^{0} f_{\alpha}\left(r_{1}, r_{2} \mid s=-A\right) d r_{2} d r_{1}
\end{aligned}
$$

where $a_{o}=\sqrt{\gamma^{2}+A^{2}}$ and $f_{\alpha}\left(r_{1}, r_{2} \mid s=-A\right)$ is the conditional joint distribution of the received samples. For the case when $\alpha=1$,

$$
f_{\alpha}\left(r_{1}, r_{2} \mid s=-A\right)=\frac{\gamma^{2}}{\pi^{2}} \prod_{i=1}^{2} \frac{1}{\gamma^{2}+\left(r_{i}+A\right)^{2}}
$$

Assuming that the transmitted symbols are equally likely, the average probability of error is:

$$
P_{e}=1-\left(P_{c \mid-A}\right)
$$

The double integral in (4.10) can be reduced to a single integral, which can be simply evaluated numerically, by applying the following identity:

$$
\begin{aligned}
& \int_{r_{1}=a}^{b} \int_{r_{2}=f_{1}\left(r_{1}\right)}^{f_{2}\left(r_{2}\right)} f_{\alpha}\left(r_{1}, r_{2} \mid s=-A\right) d r_{2} d r_{1} \\
= & \frac{\gamma^{2}}{\pi^{2}} \int_{r_{1}=a}^{b} \frac{\tan ^{-1}\left[\frac{A+f_{2}\left(r_{1}\right)}{\gamma}\right]-\tan ^{-1}\left[\frac{A+f_{1}\left(r_{1}\right)}{\gamma}\right]}{\gamma\left[\gamma^{2}+\left(r_{1}+A\right)^{2}\right]} d r_{1} .
\end{aligned}
$$




\section{Gaussian Detector}

The probability of correct decision for the Gaussian detector can be calculated analytically by changing the decision boundaries in (4.10):

$$
\begin{aligned}
P_{c \mid-A}= & \iint_{Z} f_{\alpha}\left(r_{1}, r_{2} \mid s=-A\right) d r_{2} d r_{1} \\
= & 2 \times \int_{r_{1}=0}^{\infty} \int_{r_{2}=-\infty}^{-r_{1}} f_{\alpha}\left(r_{1}, r_{2} \mid s=-A\right) d r_{2} d r_{1}+ \\
& \int_{r_{1}=-\infty}^{0} \int_{r_{2}=-\infty}^{0} f_{\alpha}\left(r_{1}, r_{2} \mid s=-A\right) d r_{2} d r_{1} .
\end{aligned}
$$

Then, the probability of error can be calculated using (4.12).

\section{Piecewise Detector}

To evaluate the performance of the piecewise detector, the decision regions in Figure 4.11 are used to construct the integration limits of the joint probability density function in (4.10). So the conditional probability of correct decision of the piecewise detector is:

$$
\begin{aligned}
P_{c \mid-A}= & 2 \times \int_{r_{1}=0}^{a_{o}} \int_{r_{2}=m r_{1}-(m+1) a_{o}}^{-r_{1}} f_{\alpha}\left(r_{1}, r_{2} \mid s=-A\right) d r_{2} d r_{1}+ \\
& 2 \times \int_{r_{1}=a_{o}}^{\left(1+\frac{1}{m}\right) a_{o}} \int_{r_{2}=-r_{1}}^{m r_{1}-(m+1) a_{o}} f_{\alpha}\left(r_{1}, r_{2} \mid s=-A\right) d r_{2} d r_{1}+ \\
2 \times \int_{r_{1}=-\infty}^{0} \int_{r_{1}=\left(1+\frac{1}{m}\right) a_{o}}^{\infty} f_{\alpha}\left(r_{1}, r_{2} \mid s=-r_{1}\right. & \int_{r_{2}=-\infty}^{0} f_{\alpha}\left(r_{1}, r_{2} \mid s=-A\right) d r_{2} d r_{1}+
\end{aligned}
$$


The performance of the piecewise detector is compared to the optimal and Gaussian detectors and validated by simulation results represented next.

\section{Simulation Results and Discussion}

To evaluate the performance of the proposed detector, the probability of error of the optimal and suboptimal piecewise detector is evaluated by simulation for the case when $\alpha=0.7,1,1.5$ and 1.9. The simulation setup is the same as used in Chapter 3. The simulation results validate the analytic performance of the piecewise detector in case when $\alpha=1$, and demonstrate, the performance when $\alpha \neq 1$.

Figure 4.13 shows the performance of the piecewise detector with $m=1$ and $m=m_{\text {opt }}$ when $\alpha=1$. It can be seen that the performance of the proposed piecewise detector approaches the optimal one with much less complexity. Also, when $m=1$ is used, the performance of the piecewise detector is only $0.2 \mathrm{~dB}$ less than the optimal one at a BER of $4 \times 10^{-3}$. When $m=m_{\text {opt }}$ is used, the performance of the piecewise detector becomes closer to the optimal performance because it has the minimum excess probability of error, which is consistent with the analysis in Section 4.4.2.1.

Although the proposed piecewise detector is designed for the case when $\alpha=1$, it shows robust performance for different values of $\alpha$. Figure 4.14 and Figure 4.15 show the near optimal performance of the proposed detector for $\alpha=0.7$ and 1.5, respectively. Figure 4.16 shows the performance when $\alpha=1.9$. It can be seen that the performance of the piecewise detector approaches the optimal performance at higher GSNR, however, it is almost $1 \mathrm{~dB}$ less that the optimal at lower GSNR. This performance is expected because, at higher GSNR, the optimal decision boundaries when $\alpha$ approaches 2 become closer to the case when $\alpha=1$, as shown in Figure 4.6, so the optimal boundaries can be well approximated by (4.4). However, this approximation becomes loose at lower values of GSNR, which results in performance degradation of the piecewise detector. This small degradation could be improved by adjusting the piecewise detector parameters such as the cross point, $a_{o}$, and the slope $m$ to give a 
better approximation of the optimal boundaries when $\alpha \neq 1$, if so desired.

Finally, it is worth reiterating that this proposed technique is only applicable to the case when $N=2$. It is difficult to generalize the technique for $N>2$ because the decision regions become more complex. Figure 4.17 and 4.18 show the optimal decision regions for $N=3$. It can be seen that how difficult it is to approximate the decision boundaries in the three-dimensional case.

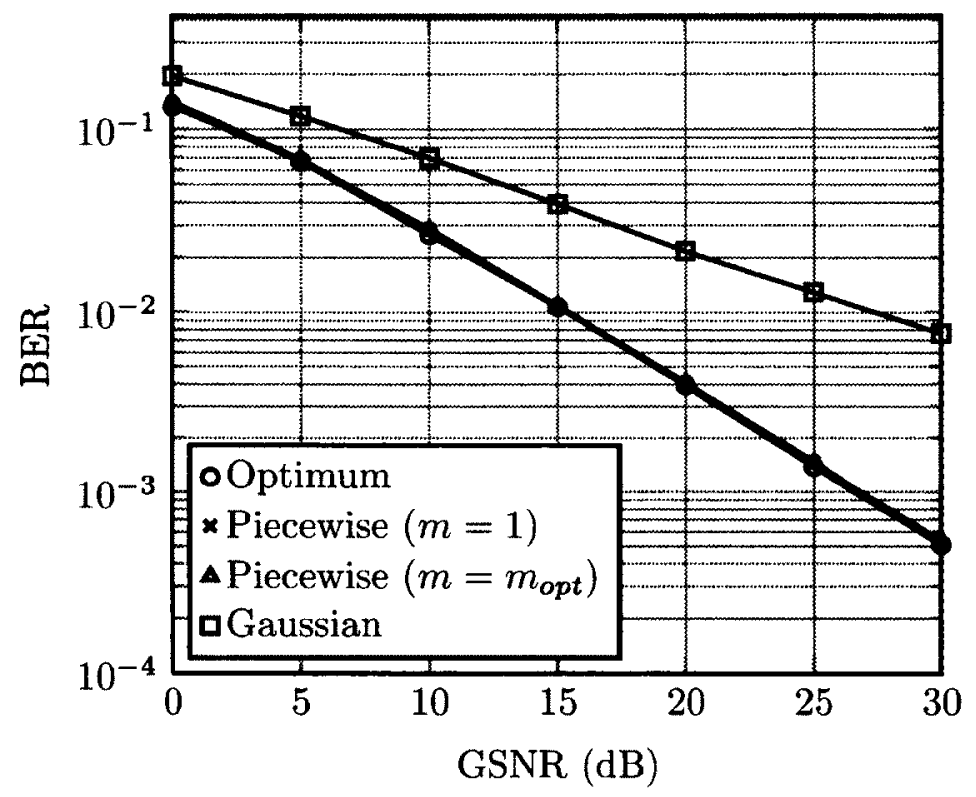

Figure 4.13: Analytical and simulated probability of error with $\alpha=1$. Analytic: solid lines, simulations: markers only. 


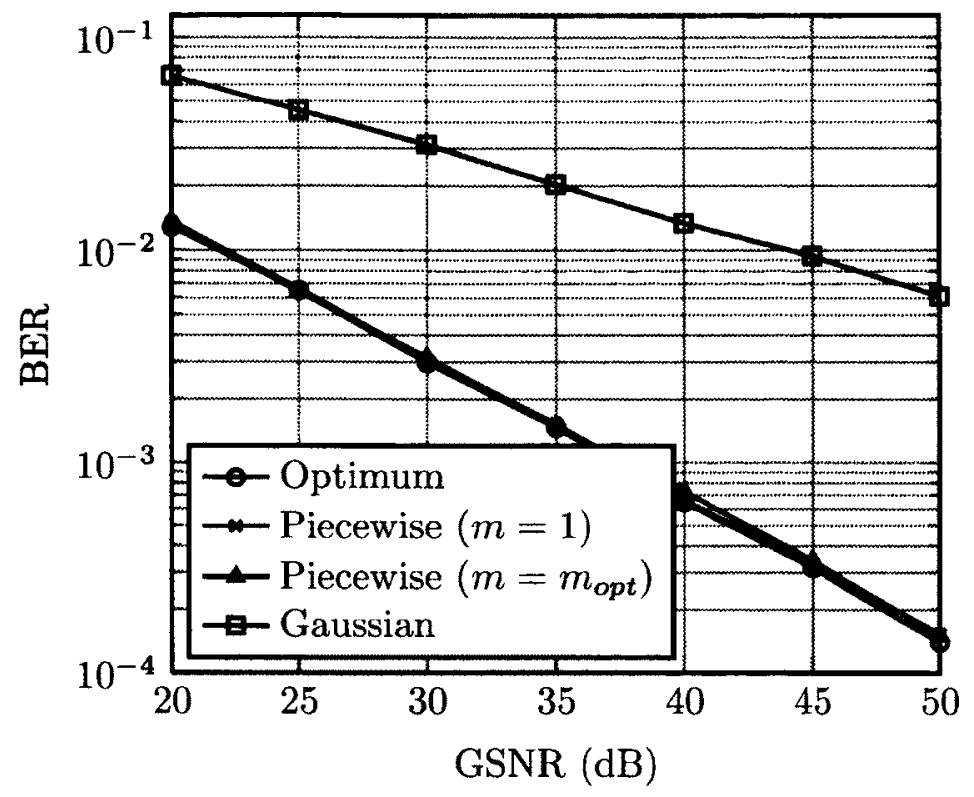

Figure 4.14: Performance comparison with $\alpha=0.7$.

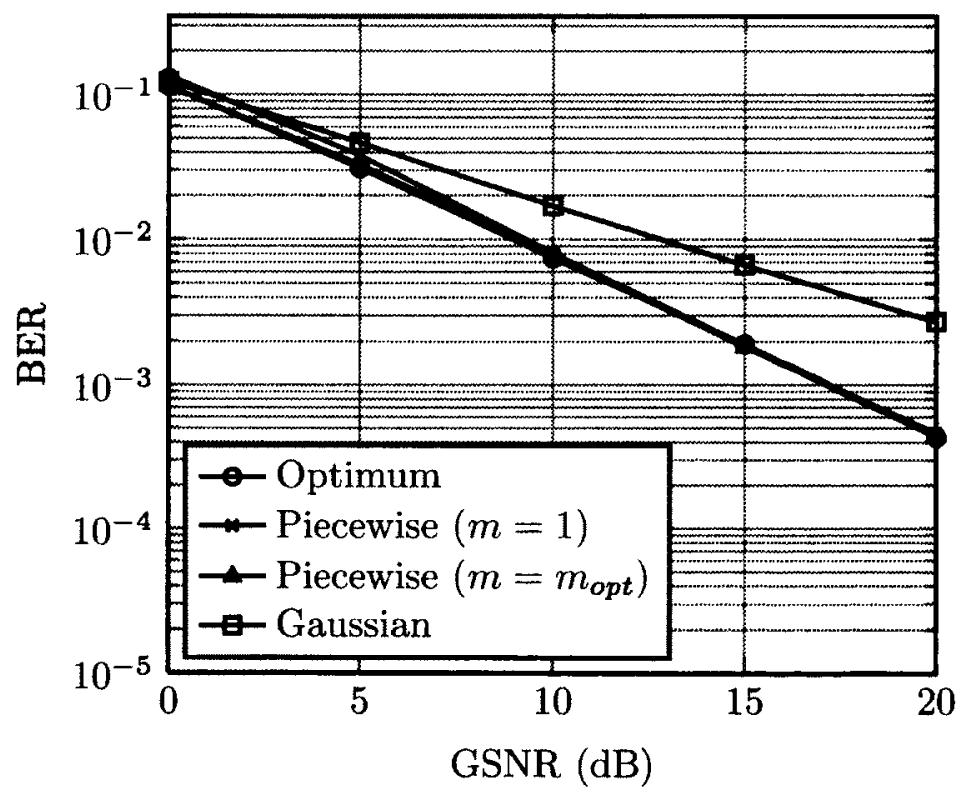

Figure 4.15: Performance comparison with $\alpha=1.5$. 


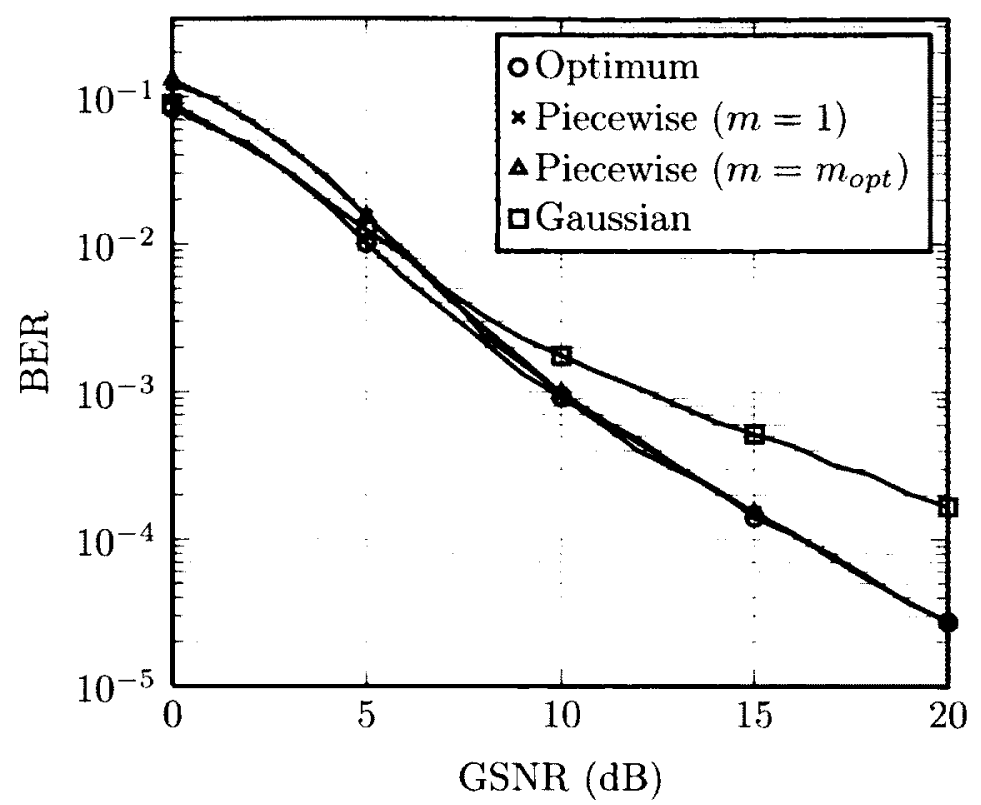

Figure 4.16: Performance comparison with $\alpha=1.9$.

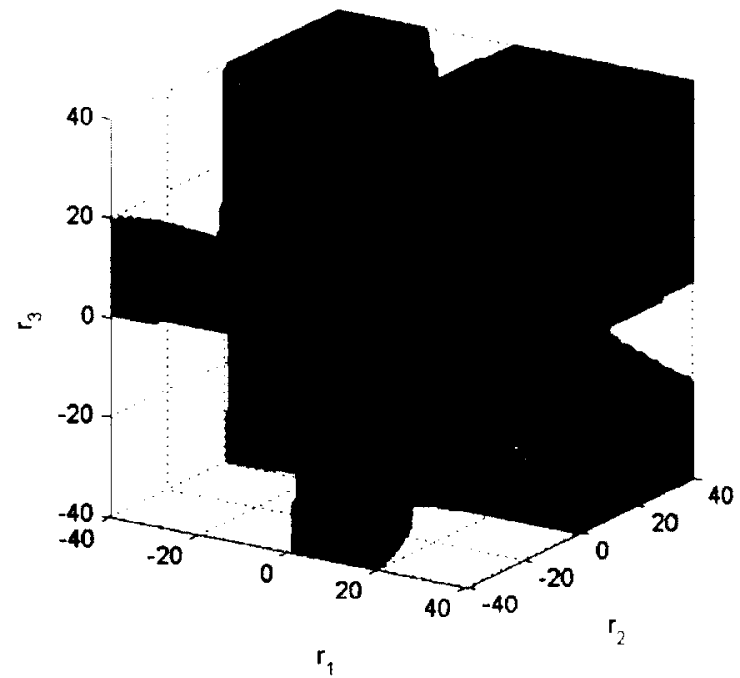

Figure 4.17: Optimal decision regions for $N=3$ and $\alpha=1$. 


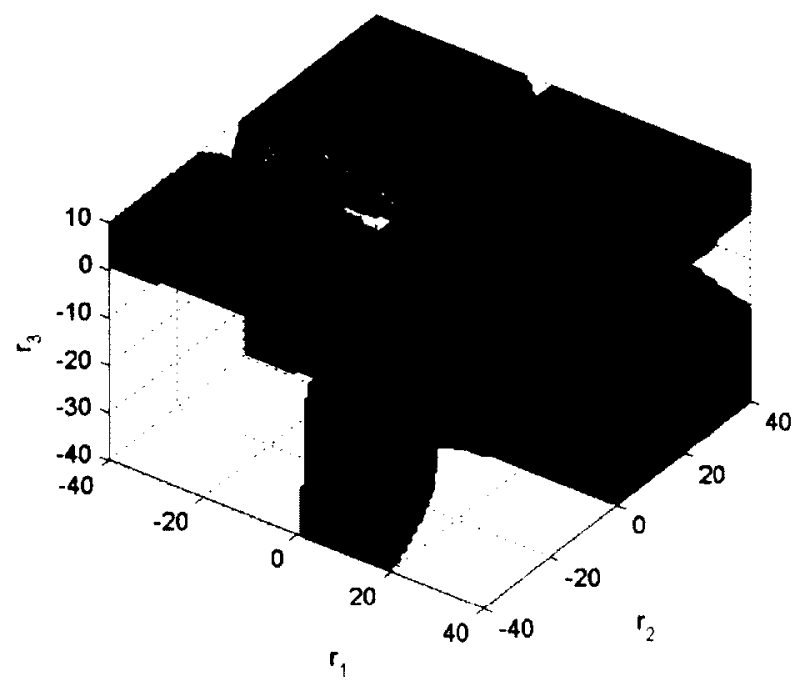

Figure 4.18: A cross section in the optimal decision regions for $N=3$ and $\alpha=1$.

\subsection{Conclusion}

A new framework is proposed to analyze the behavior of the optimal and suboptimal signal detectors in $\mathrm{S} \alpha \mathrm{S}$ noise. The proposed framework calculates the optimal decision regions and compares them to those of the suboptimal detectors. Based on this analysis, an accurate justification of the structure and the performance of several suboptimal detectors is presented. This analysis has been used to propose the adaptive soft limiter detector which gives near optimal performance for large $N$ with low-complexity. Also, a piecewise linear detector has been proposed to give nearoptimal performance for the case when $N=2$. However, it is difficult to generalize the piecewise detector for $N>2$ because the optimal decision regions become more challenging to approximate. In the next chapter, we will present a simple general approach that can be used to design simple near-optimal detectors for any value of $N$ and $\alpha$. This approach can be also used to design several elements in the receiver such as the diversity combiner and different types of decoders. 


\section{Chapter 5}

\section{Log-likelihood Ratios: A Generalized Framework to Design Suboptimal Detectors for Signals in SaS Noise}

\subsection{Introduction}

In the previous chapter, we proposed the adaptive soft limiter (ASL) with nearoptimal performance for large $N$ which suggests that the ASL detector may approximate the optimal decision regions. However, for lower values of $N$, the ASL detector gives little performance improvement over the Gaussian detector, which is a result of an inaccurate approximation of the optimal decision regions. A piecewise linear detector was also proposed for the case when $N=2$ with performance almost the same as the optimal one with low complexity. It seems that approximating the optimal decision boundaries is a good way to achieve near-optimal performance; however, it is too difficult to generalize the piecewise approximation method for $N>2$ due to the increasing complexity of the optimal decision regions.

In this chapter, we propose to apply a piecewise linear approximation to the 
optimal log-likelihood ratios of the received samples

$$
\begin{aligned}
L L R & =\log \left\{\frac{\prod_{k=1}^{N} f_{\alpha}\left(r_{k}-A\right)}{\prod_{k=1}^{N} f_{\alpha}\left(r_{k}+A\right)}\right\} \\
& =\sum_{k=1}^{N} L L R_{O p t}\left(r_{k}\right)
\end{aligned}
$$

where

$$
L L R_{O p t}\left(r_{k}\right)=\log \left\{\frac{f_{\alpha}\left(r_{k}-A\right)}{f_{\alpha}\left(r_{k}+A\right)}\right\}
$$

Now, instead of approximating multi-dimensional decision boundaries, the problem reduces to only approximating $L L R_{O p t}\left(r_{k}\right)$ which is a one-dimensional function.

Another motivation behind the proposal of approximating the optimal LLR is the use of the locally optimal detector. As shown in Section 4.3.5, the optimal Bayesian detector, which implements the optimal likelihood ratios in (3.2), has been approximated by the locally optimal detector under the assumption of weak signals [55]. Moreover, the locally optimal detector has been approximated by many other simple nonlinearities to overcome the complexity of the locally optimal nonlinearity. In this thesis, we choose to directly approximate the optimal nonlinearity, which is simply the optimal log-likelihood ratios (LLRs). In this way, we get rid of one stage of approximation. On one hand, the use of the approximated LLRs will not be limited by the weak signal assumption. On the other hand, the optimal LLRs is another way to represent the optimal decision boundaries. So, by using a good approximation of the optimal LLRs, we provide a simple way to get a good fit of the optimal decision regions for any values of $N$, which will be reflected on the detector's performance. 


\subsection{Piecewise Linear LLR Approximation}

Figures 5.1 to 5.4 show the optimal LLR for different values of $\alpha$ at a given value of GSNR. Also, Figures 5.5 to 5.9 show how the optimal LLR changes with GSNR at a given value of $\alpha$. When $\alpha=2$ (the Gaussian case), the optimal LLR is linear; however, when $\alpha<2$, the optimal LLR behaves differently, as it goes to zero when $r \rightarrow \infty$, due to the heavy tail of the noise distribution. This behavior can be explained by the decaying property of the distribution's tail. As shown in (2.5) and (2.7), for $\alpha<2$, the tail decays polynomially with a rate much slower than that of the exponentially decaying tail for the case when $\alpha=2$. The slow decay of the distribution tail results in a little difference between the nominator and denominator of the likelihood ratio, which becomes almost equal when $r \rightarrow \infty$. Also, it can be shown in the figures that when the tail becomes heavy, i.e., the rate of the tail decay is slow (e.g. polynomial), the LLR tends to go to zero. However, when the rate of the tail decay is fast (e.g. exponential), the LLR tends to go to infinity. The optimal LLR has a closed form expression only when $\alpha=2$ and $\alpha=1$ as follows

$$
\begin{gathered}
L L R_{\text {Gauss }}\left(r_{k}\right)=\frac{2 A}{\sigma^{2}} r_{k} \\
L L R_{\text {Cauchy }}\left(r_{k}\right)=\log \frac{\gamma^{2}+\left(r_{k}+A\right)^{2}}{\gamma^{2}+\left(r_{k}-A\right)^{2}}
\end{gathered}
$$




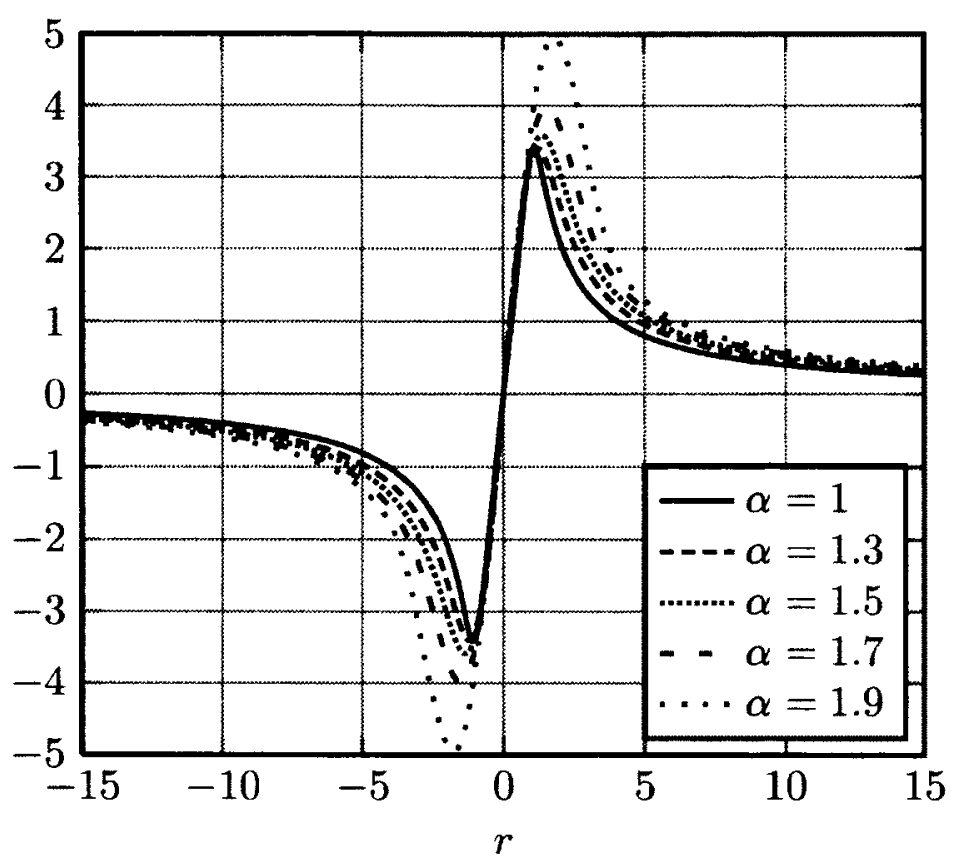

Figure 5.1: Optimal LLR with GSNR $=0 \mathrm{~dB}$.

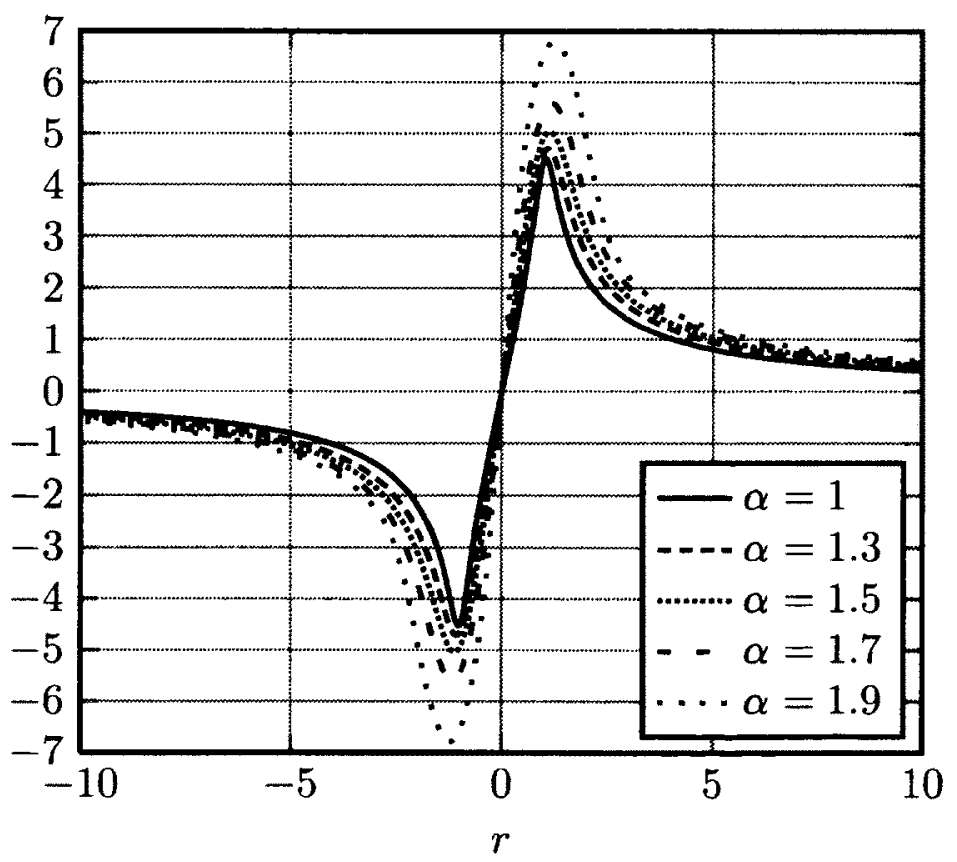

Figure 5.2: Optimal LLR with GSNR $=5 \mathrm{~dB}$. 


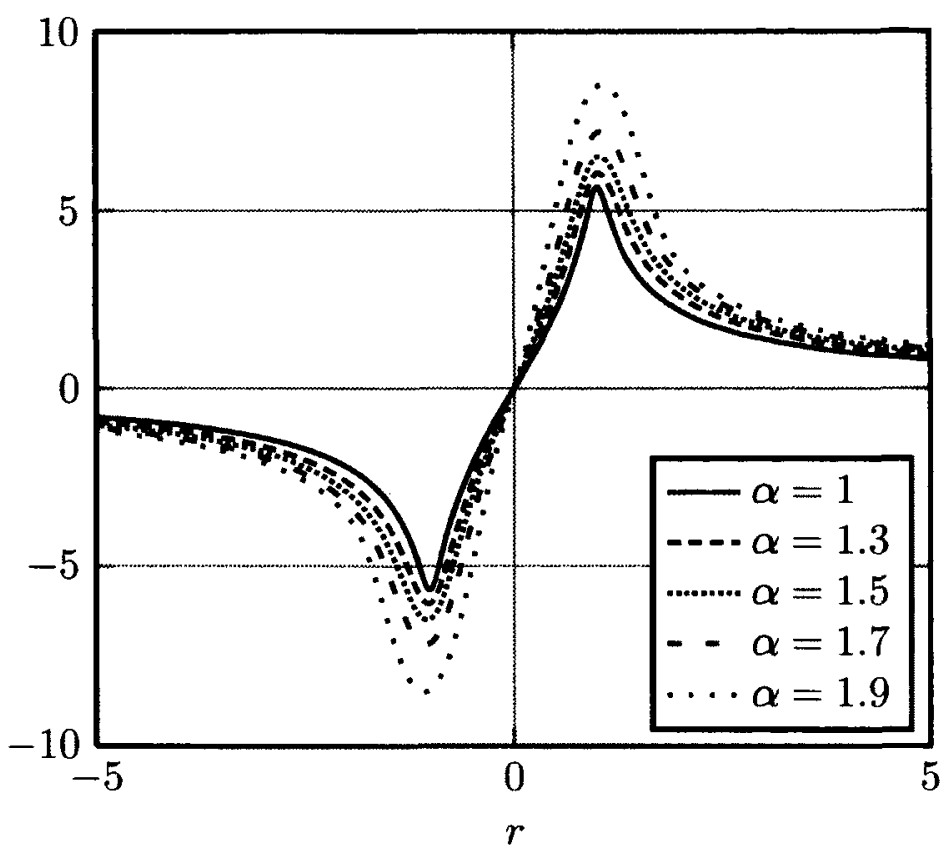

Figure 5.3: Optimal LLR with GSNR $=10 \mathrm{~dB}$.

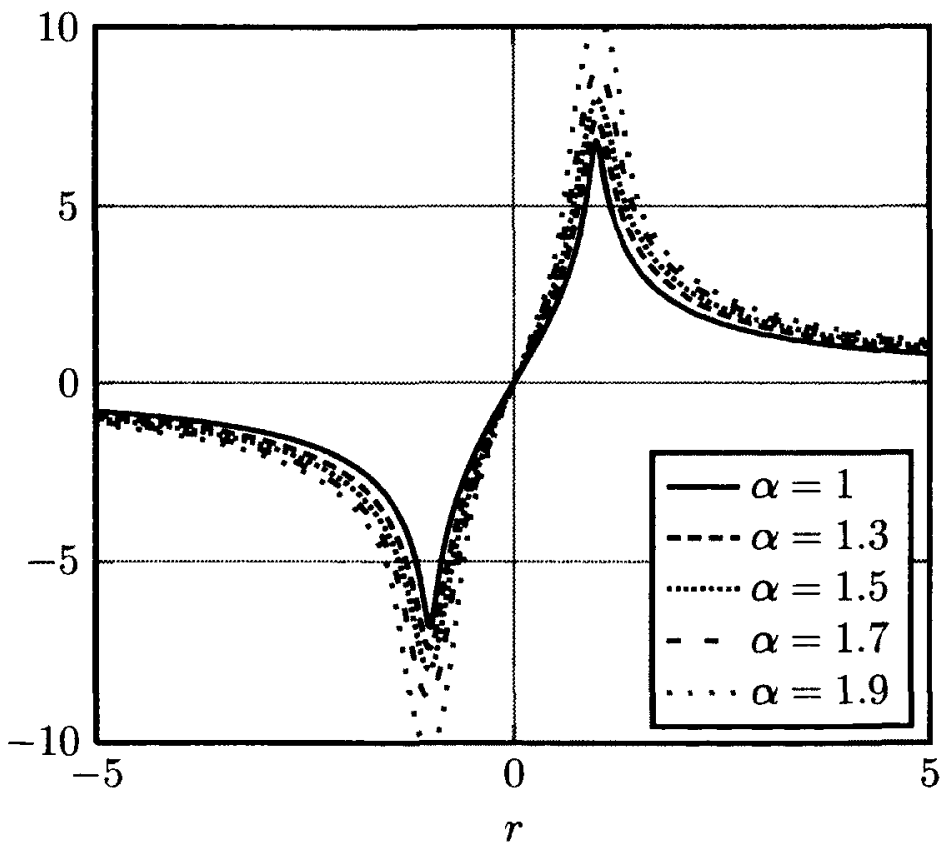

Figure 5.4: Optimal LLR with GSNR $=15 \mathrm{~dB}$. 


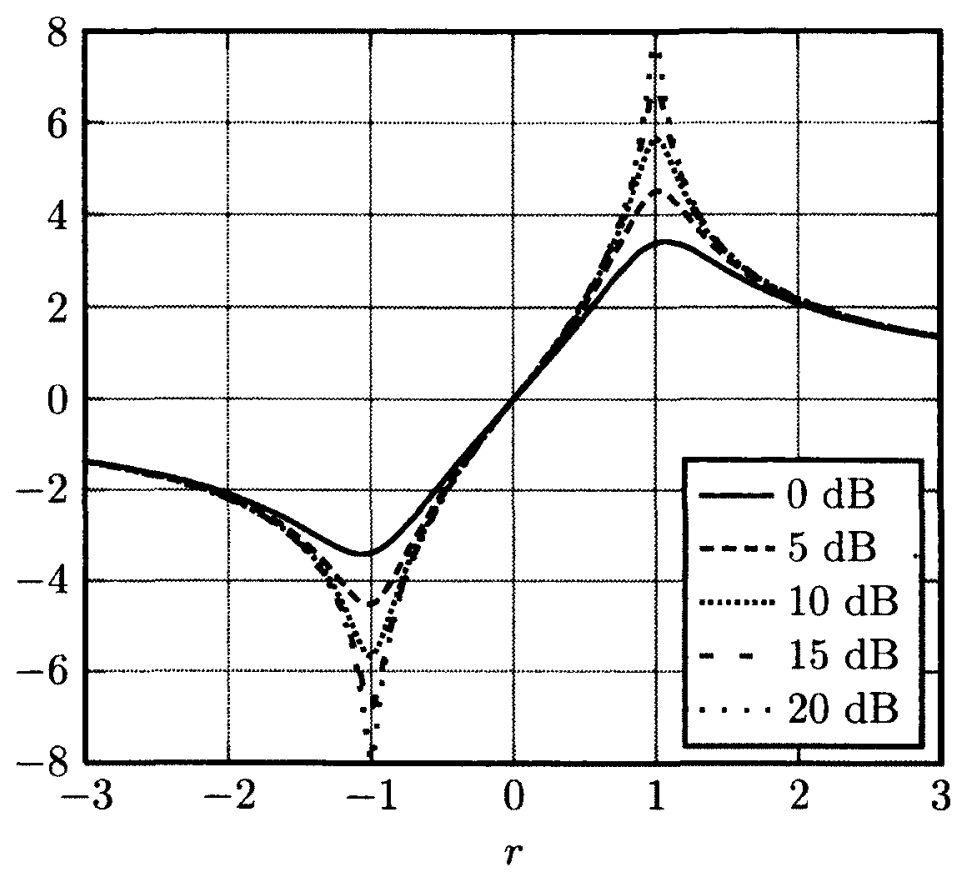

Figure 5.5: Optimal LLR for different values of GSNR with $\alpha=1$.

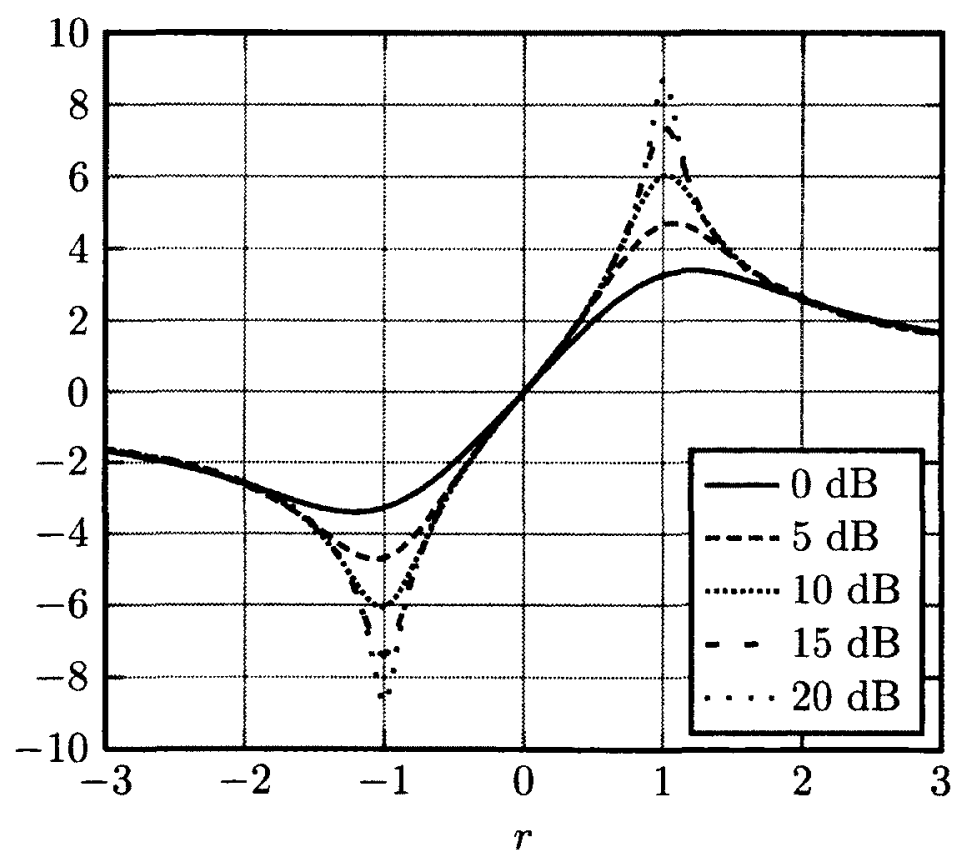

Figure 5.6: Optimal LLR for different values of GSNR with $\alpha=1.3$. 


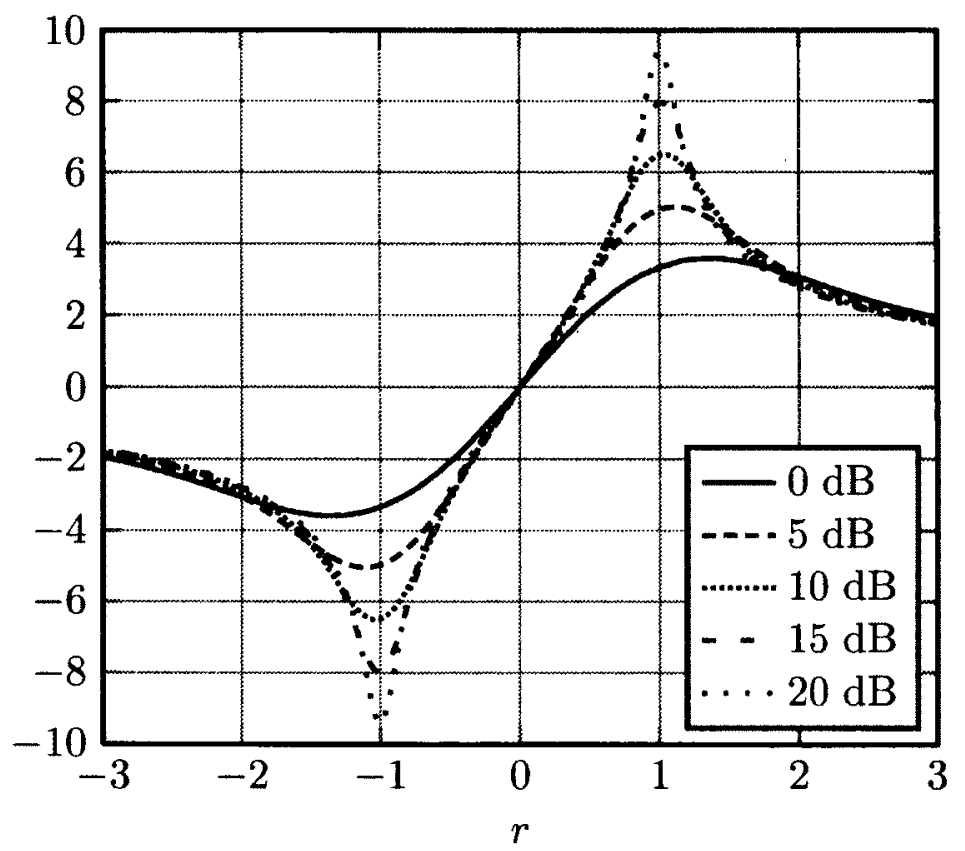

Figure 5.7: Optimal LLR for different values of GSNR with $\alpha=1.5$.

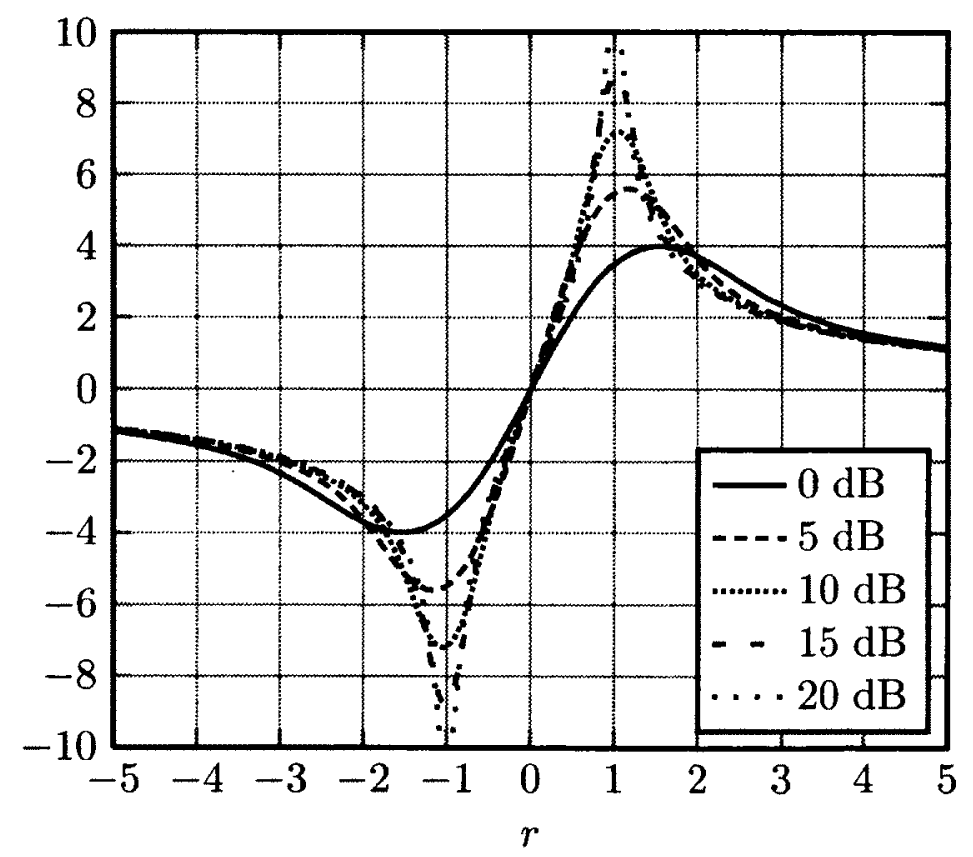

Figure 5.8: Optimal LLR for different values of GSNR with $\alpha=1.7$. 


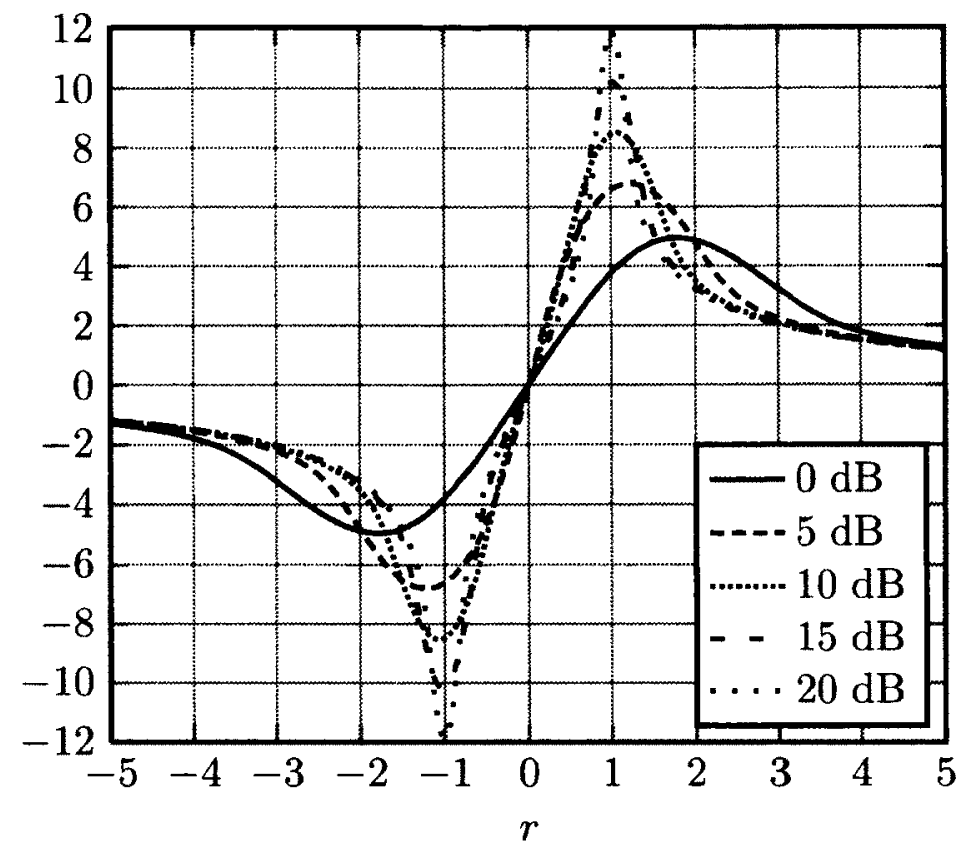

Figure 5.9: Optimal LLR for different values of GSNR with $\alpha=1.9$.

We propose to approximate the optimal LLR by using simple piecewise linear segments. Due to the antisymmetry of the LLR, we consider only the positive range of the received signal, $r$, which is divided into three regions according to the rate of the change of the optimal LLR. The proposed piecewise linear LLR (PWL-LLR), as shown in Figure 5.10, is completely defined by two coordinate-points $(a, c)$ and $(b, d)$ as follows,

$$
L L R_{P W L}\left(r_{k}\right)= \begin{cases}\frac{c}{a} r_{k} & r_{k} \leq a \\ c+m(r-a) & a<r_{k} \leq b \\ d & r_{k}>b\end{cases}
$$

where $m=\frac{d-c}{b-a}$, and $a, b, c$ and $d$ are the fitting parameters which are optimized by minimizing the mean square error (MSE) of the fitting as follows

$$
a, b, c, d=\arg \min \left[\int_{0}^{\infty}\left[L L R_{O p t}(r)-L L R_{P W L}(r)\right]^{2} f(r) d r\right]
$$


where $f(r)$ is the pdf of the received samples,

$$
f(r)=f(r \mid s=A) p(s=A)+f(r \mid s=-A) p(s=-A) .
$$

and $L L R_{O p t}$ and $L L R_{P W L}$ are given in (5.3) and (5.6), respectively. It is mathematically difficult to minimize the MSE in (5.7) due to the complexity of $L L R_{O p t}(r)$. However, we propose to use a simple yet efficient approximation of the fitting parameters $a$ and $c$. First, we use the pair $(\tilde{a}, \tilde{c})$ to be the peak of the optimal LLR. Then, we calculate the conditionally optimal values of $\tilde{b}$ and $\tilde{d}$ (conditioned on $a=\tilde{a}$ and $c=\tilde{c}$ ) by minimizing the MSE in (5.7) numerically.

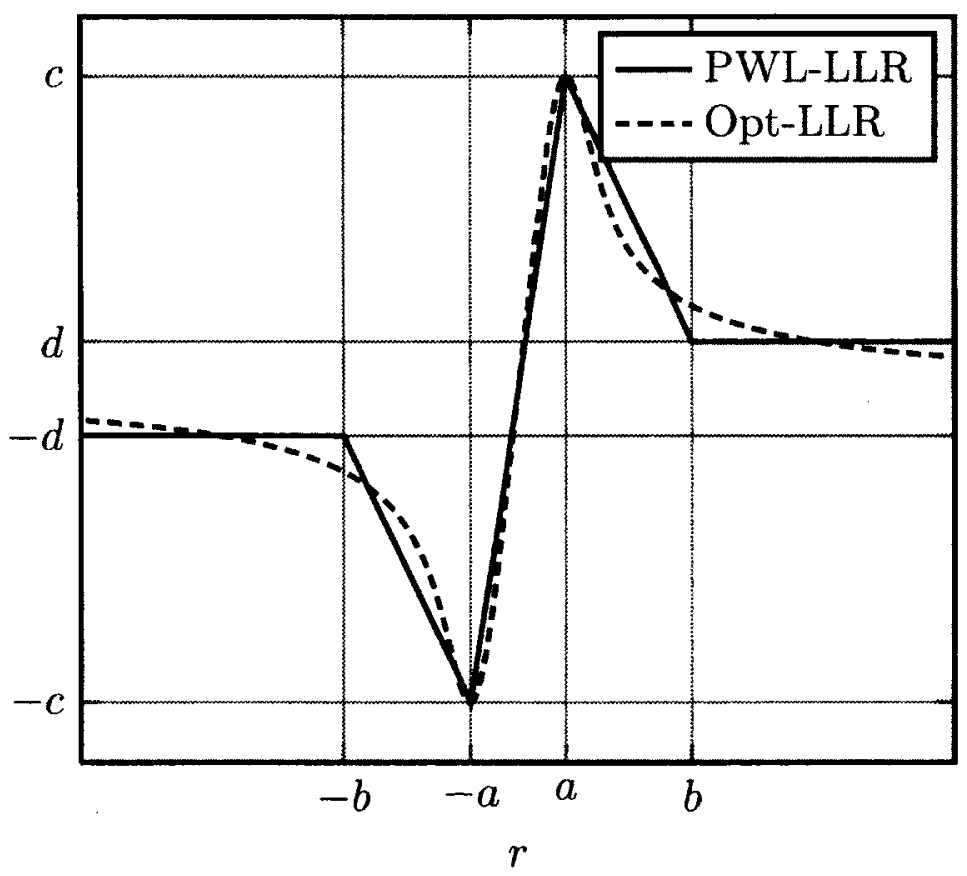

Figure 5.10: The piecewise linear LLR (PWL-LLR).

Because of the lack of a closed form expression of the optimal LLR, there is no analytical expression to calculate the peak of the LLR for the case when $\alpha \neq 1$. So, we use the fitting of the numerically calculated $\tilde{a}$ and $\tilde{c}$ to get a simple formula to 
calculate $\tilde{a}$ as a linear function of $\alpha$ and $\tilde{c}$ as a quadratic function of $\alpha$ as follows

$$
\tilde{a}=a_{1} \alpha+a_{0}
$$

where

$$
\begin{aligned}
& a_{1}=0.775194 \psi^{-2.43491 / \ln (10)} \\
& a_{0}=0.993484-0.758831 \psi^{-2.54349 / \ln (10)}
\end{aligned}
$$

and

$$
\tilde{c}=c_{2} \alpha^{2}+c_{1} \alpha+c_{0}
$$

where

$$
\begin{aligned}
& c_{2}=\left(3.46366-8.48571 e^{-4} \psi\right) \\
& c_{1}=(-8.43602+0.130997 \psi) \\
& c_{0}=(8.43171+0.0974844 \psi)
\end{aligned}
$$

and $\psi$ is the GSNR in a linear scale as defined in 2.11. Figures 5.11 and 5.12 shows the numerically calculated and the approximated values of $\tilde{a}$ and $\tilde{c}$, respectively. The conditional optimal value $\tilde{b}$ and $\tilde{d}$ are obtained by numerically minimizing the MSE in (5.7) conditioned on $a=\tilde{a}$ and $c=\tilde{c}$. Figures 5.13 to 5.16 shows $\tilde{b}$ and $\tilde{d}$, normalized to $\tilde{a}$ and $\tilde{c}$, respectively, for different values of $\alpha$ and GSNR. It can be seen that the optimal values of $b$ and $d$ can be easily approximated linearly as a function of $\alpha$ for different values of GSNR. 


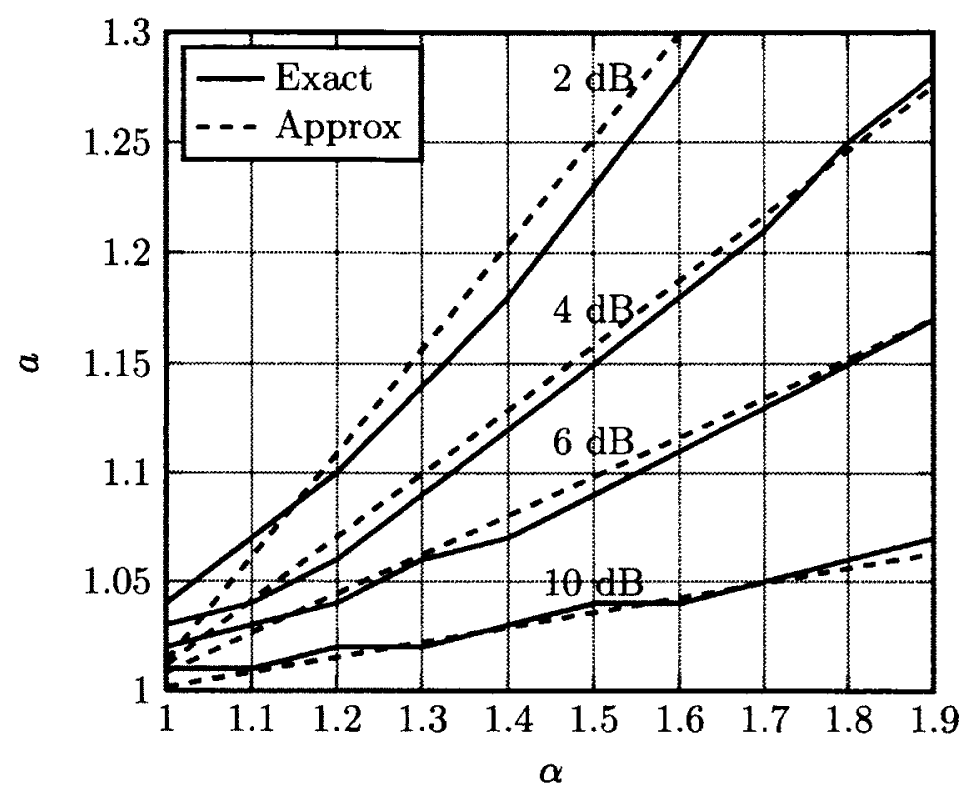

Figure 5.11: Exact and approximate values of $a$.

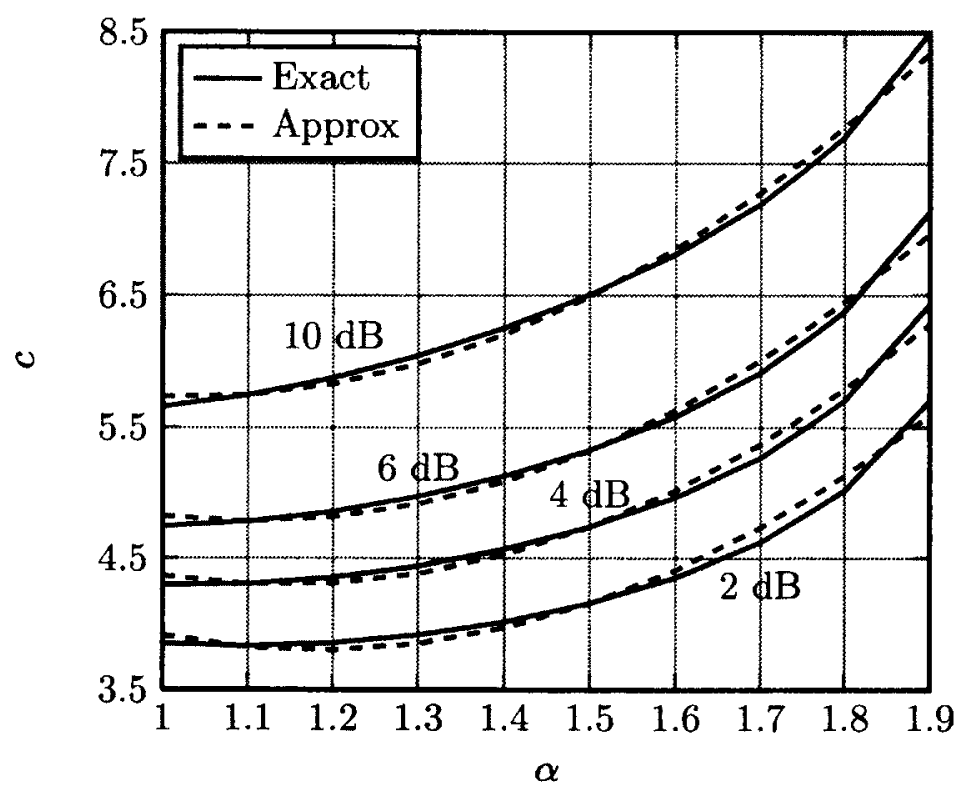

Figure 5.12: Exact and approximate values of $c$. 


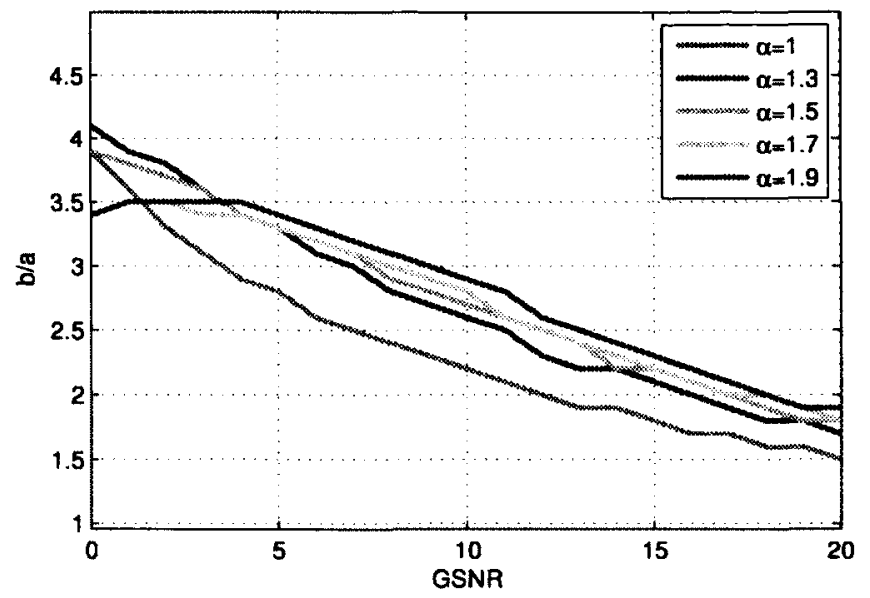

Figure 5.13: Optimal values of $b$ for different values of $\alpha$.

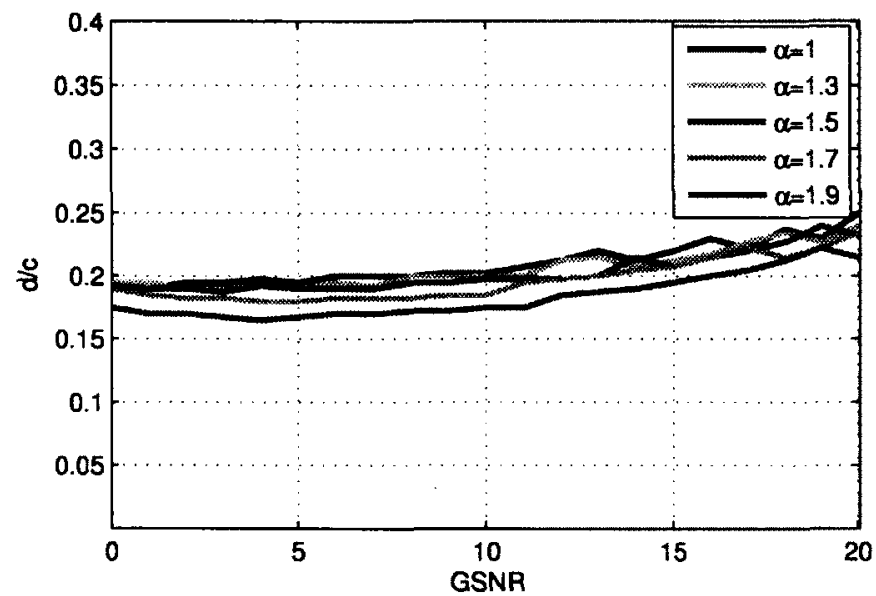

Figure 5.14: Optimal values of $d$ for different values of $\alpha$. 


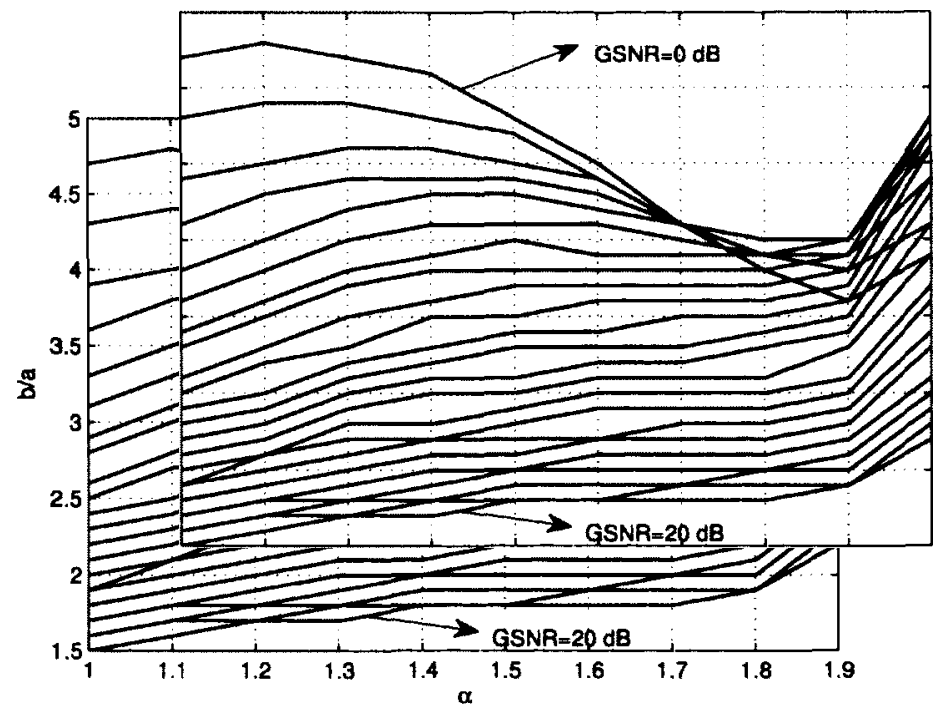

Figure 5.15: Optimal values of $b$ for different values of GSNR.

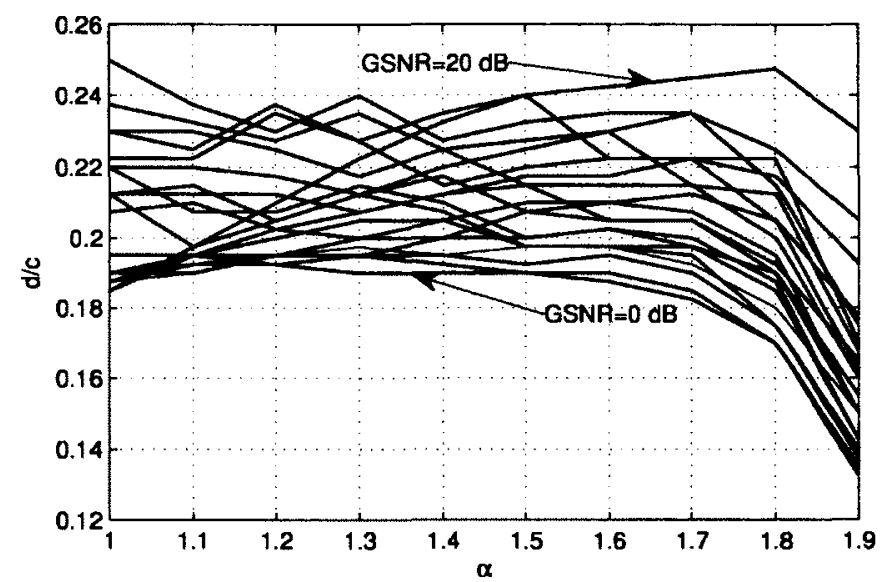

Figure 5.16: Optimal values of $d$ for different values of GSNR.

\subsubsection{A Simplified Approximation of the PWL-LLR Param- eters}

In the previous section, we have used linear and quadratic fitting to get close approximations to the values of optimal parameters of the PWL-LLR. To further simplify the calculations of those parameters, we choose to use the coordinates of the peak 
of the Cauchy LLR as an approximation of $(a, c)$, as can be seen in Figures 5.1 to 5.4. Also, from Figure 5.11, we can see that the exact values of the parameter $a$ with different values of $\alpha$ are close to the value of $a$ when $\alpha=1$ for moderate and high values of GSNR.

From Figure 5.13, we can see that when the GSNR increases, $b$ gets closes to $a$ such that $a \leq b<4 a$. Note that when $b=a$, the PWL-LLR becomes similar to the nonlinearity used by the proposed adaptive soft-limiter (ASL) detector. Simulation results show no significant difference when using $b=2 a$ and $b=3 a$.

For the values of $c$ and $d$, we use the Cauchy LLR formula to calculate their values given $a$ and $b$, respectively. So, the PWL-LLR parameters are calculated as follows:

$$
\begin{aligned}
& \tilde{a}=\sqrt{\gamma^{2}+A^{2}} \\
& \tilde{c}=\log \frac{\gamma^{2}+(a+A)^{2}}{\gamma^{2}+(a-A)^{2}} \\
& \tilde{b}=2 a \\
& \tilde{d}=\log \frac{\gamma^{2}+(b+A)^{2}}{\gamma^{2}+(b-A)^{2}}
\end{aligned}
$$

\section{Complexity of the test statistic}

To calculate the test statistic accroding to 5.6 , we need

- $2 N$ multiplications.

- $2 N$ additions.

- $2 N$ comparators.

The parameters of the PWL-LLR, $m$ and $c$, depend only on $\gamma$ and $A$ which are assumed to be constant and known to the receiver. Those parameters need

- $1 \log$ operations.

- 1 additions.

- 2 multiplications.

- 2 divisions. 


\subsubsection{Decision Boundaries of PWL-LLR Detector}

To justify the performance of the PWL-LLR detector, the decision regions of the proposed detector are compared with the optimal ones. For simplicity, and as a proof of concept, we will present the case when $N=2$. The PWL-LLR decision boundaries can be calculated analytically for the case when $N=2$. Figure 5.17 shows the decision boundaries of the PWL-LLR detector which closely fit the optimal ones. Furthermore, the decision boundaries of the PWL-LLR are consistent with the boundaries of the piecewise detector proposed in Chapter 4, except fot the hard limiter boundaries which results from the approximation of the optimal LLR by a constant value, $d$.

Also, Figure 5.18 shows the decision regions of the PWL-LLR detector with $N=3$, which closely approximate the optimal decision regions shown in Figure 4.17. So, the proposed PWL-LLR detector provides a general way of designing simple suboptimal detectors for any value of $N$.

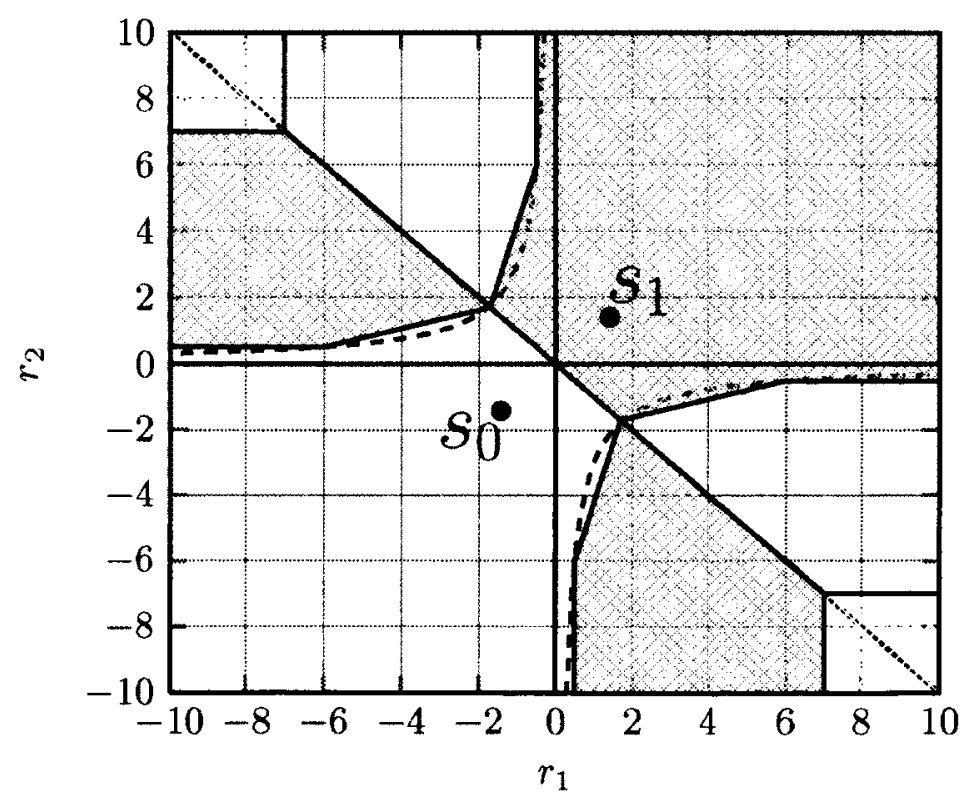

Figure 5.17: Decision boundaries of the PWL-LLR detector compared to the optimal ones with $\alpha=1, N=2$. Solid: Optimal, Dashed: PWL LLR. 


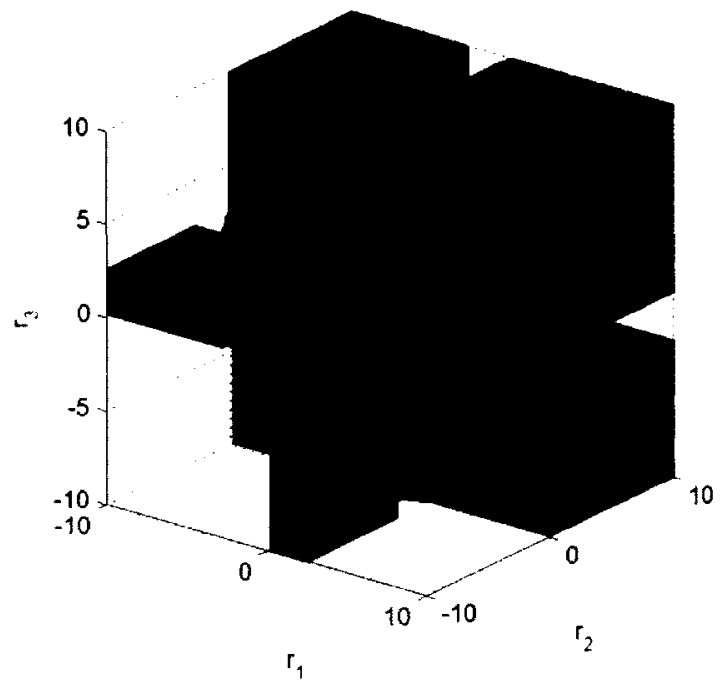

Figure 5.18: Decision regions of the PWL-LLR with $\alpha=1$ and $N=3$.

\subsubsection{Performance Evaluation of the PWL-LLR Detector}

The performance of the PWL-LLR detector is compared to the ASL, the Cauchy, and the optimal detectors for different values of $\alpha$ and $N$ with the same simulation setup as in section 3.4.2. 


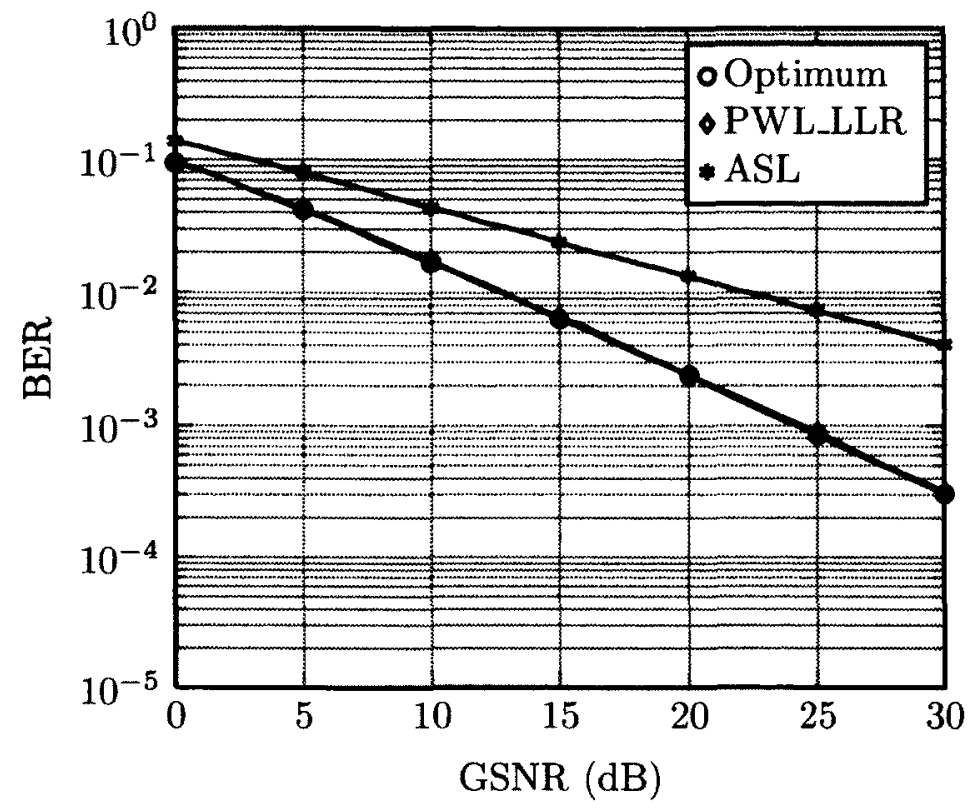

Figure 5.19: Performance comparison of the PWL-LLR detector with $\alpha=1, N=2$.

Figure 5.19 shows the performance of the PWL-LLR detector when $\alpha=1$ (where Cauchy detector is the optimal) and $N=2$. As can be seen in the figure, the PWLLLR gives near-optimal performance due to the close fit of the optimal LLR and consequently the optimal decision regions, especially for low values of $N$ where the ASL detector performs poorly compared to the optimal.

Figure 5.20 shows the performance of the PWL-LLR detector when $\alpha=1$ and $N=10$. It can be shown that, for higher values of $N$, the PWL-LLR detector still gives near-optimal performance. However, there is a slight performance degradation at low GSNR due to the inaccurate fit of the optimal LLR. Moreover, this slight performance degradation becomes noticeable when $N$ increases because, at higher values of $N$, the effective GSNR per sample is less than the GSNR by $10 \log _{10} N$ which explains the slight degradation in the performance with $N=10$ at low GSNR, as shown in Figure 5.20. 


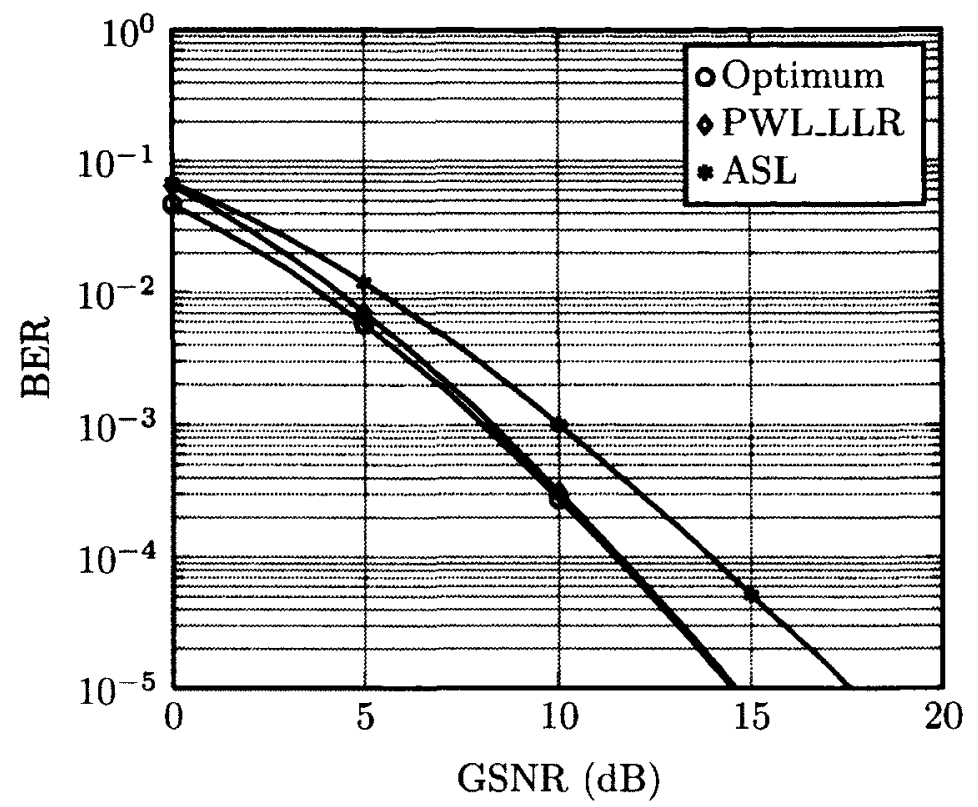

Figure 5.20: Performance comparison of the PWL-LLR detector with $\alpha=1, N=$ 10.

In Figures 5.21 and 5.22, it can be seen that the PWL-LLR detector gives nearoptimal performance for different values of $\alpha$ where $\alpha=1.5$ and $\alpha=1.9$, respectively, with $N=4$. In Figure 5.21, the PWL-LLR detector gives indistinguishable performance from optimal, while the ASL detector is around $4 \mathrm{~dB}$ behind the optimal at $\mathrm{BER}=10^{-5}$ when $\alpha=1.5$. When $\alpha=1.9$, the PWL-LLR detector is still better than the ASL detector which is around $0.5 \mathrm{~dB}$ from the optimal, as shown in Figure 5.22. 


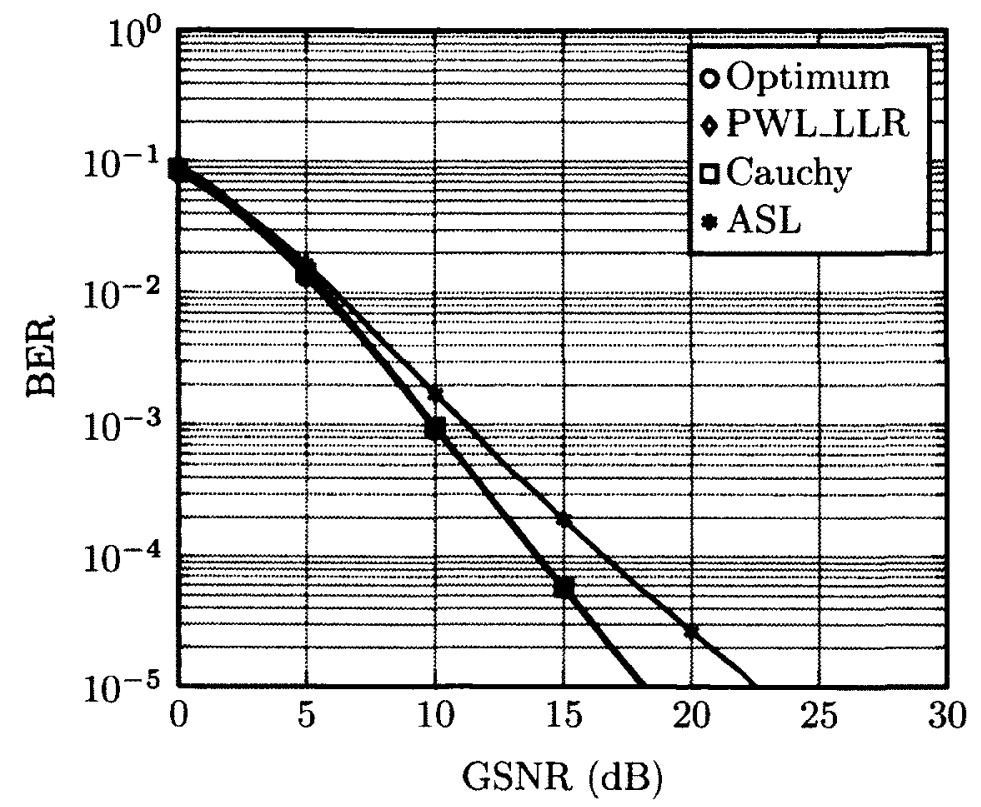

Figure 5.21: Performance comparison of the PWL-LLR detector with $\alpha=1.5$, $N=4$.

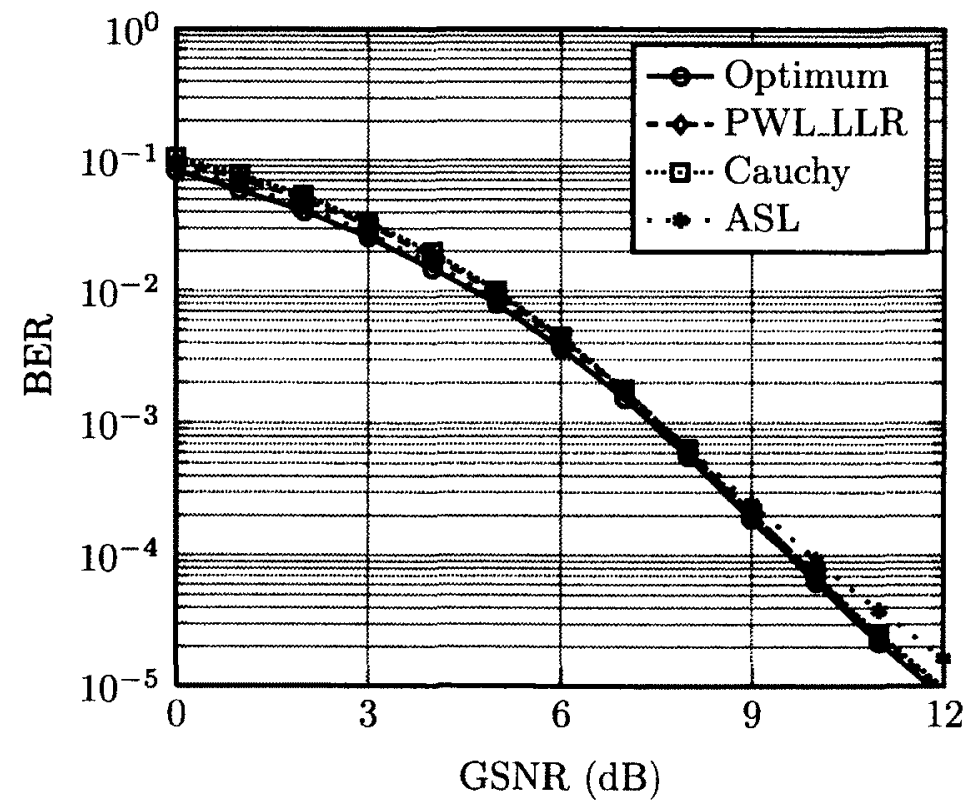

Figure 5.22: Performance comparison of the PWL-LLR detector with $\alpha=1.9$, $N=4$. 
In the above figures, we can see that the PWL-LLR has almost consistent performance compared to the ASL detector which gives near-optimal performance only with high values of $\alpha$ and moderate to high values of $N$. On the other hand, the PWL-LLR gives almost the same performance as Cauchy detector with less complexity.

\subsection{The PWL-LLR Diversity Combiner for Fading Channels}

The proposed LLR-based framework can also be extended to design a near-optimal diversity combiner for signals in $\mathrm{S} \alpha \mathrm{S}$ noise with fading channels and multiple receive antenna. A few suboptimal detectors have been proposed for fading channels, however, their performance is far from optimal. Also, they use different ad hoc simplifications with no clear justification. In this section, we will review some of these existing suboptimal detectors. We will also propose the PWL-LLR diversity combiner which gives near-optimal performance with low complexity compared to the other detectors. To evaluate the performance, we will use multiple receive antennas with uncorrelated fading on each antenna. The received signal is modeled as follows

$$
r_{k}=h_{k} s_{i}+n_{k} \quad k=1, \ldots, N
$$

where $\left\{h_{k}\right\}$ is the channel amplitude modeled with Rayleigh distribution with mean $\sigma \sqrt{\frac{\pi}{2}}$ and variance $\frac{4-\pi}{2} \sigma^{2}$, where $\sigma^{2}=\frac{1}{2}$ and $N$ is the number of the receive antennas, and $s \in\{ \pm A\}$ is the transmitted BPSK symbol. In this thesis, we assume that the amplitude of the channel coefficients, $\left\{h_{k}\right\}$, are known to the receiver. 


\subsubsection{Optimal and Suboptimal Detectors for Fading Chan- nels}

\section{Maximum Likelihood Detector}

The optimal ML detector (the optimal combiner) computes the following test statistics for a received symbol:

$$
\lambda_{M L}=\log \left\{\frac{\prod_{k=1}^{N} f_{\alpha}\left(r_{k}-h_{k} A\right)}{\prod_{k=1}^{N} f_{\alpha}\left(r_{k}+h_{k} A\right)}\right\} \underset{\substack{s_{1} \\ s_{0}}}{\gtrless} 0
$$

where $s_{0}$ is the hypothesis that $s=-A$ was transmitted, and $s_{1}$ is the hypothesis that $s=+A$ was transmitted.

\section{Maximum Ratio Combiner (MRC)}

To simplify the design of the optimal combiner, the maximum ratio combiner, which is the optimal combiner for the case when the noise is Gaussian, has been used as a benchmark for the performance of a simple combiner as in $[57,58]$ with test statistics

$$
\lambda_{M R C}=\sum_{k=1}^{N} h_{k} r_{k}
$$

\section{The Optimal Linear Combiner (OLC)}

The optimal linear combiner has been derived in [57] to calculate the optimal weights that a linear combiner can use with $\mathrm{S} \alpha \mathrm{S}$ noise, however, it is limited to the case when $1<\alpha \leq 2$. The test statistics are

$$
\lambda_{O L C}=\sum_{k=1}^{N} w_{k} r_{k}
$$


where $\left\{w_{k}\right\}$ are the optimal linear combiner weights given by

$$
w_{k}=\operatorname{sgn}\left(h_{k}\right)\left|h_{k}\right|^{\frac{1}{\alpha-1}}
$$

\section{The Sign Correlator (SC) Detector}

The sign correlator detector, which is known in many references as post detection combining (PDC), has been used in [58] with test statistics

$$
\lambda_{S C}=\sum_{k=1}^{N} \operatorname{sgn}\left(h_{k} r_{k}\right)
$$

\section{The Asymptotically Optimal (AO) Detector}

The asymptotically optimal detector has been proposed in [58] as a simplified suboptimal combiner which has a closed form expression of the test statistics

$$
\lambda_{A O}=\sum_{k=1}^{N} \log \frac{\left|r_{k}-h_{k} A\right|}{\left|r_{k}+h_{k} A\right|} .
$$

\subsubsection{The PWL-LLR Diversity Combiner}

In this section, we propose to use the PWL-LLR to design a near-optimal diversity combiner with low complexity compared to other suboptimal combiners. As explained in Section 5.2.1, the PWL-LLR is completely defined by two coordinate points $(a, c)$ 
and $(c, d)$ which are defined for optimal detection in frequency-flat fading channels as

$$
\begin{aligned}
& \tilde{a_{k}}=\sqrt{\gamma^{2}+\left(h_{k} A\right)^{2}} \\
& \tilde{c_{k}}=\log \frac{\gamma^{2}+\left(a_{k}+h_{k} A\right)^{2}}{\gamma^{2}+\left(a_{k}-h_{k} A\right)^{2}} \\
& \tilde{b_{k}}=2 a_{k} \\
& \tilde{d_{k}}=\log \frac{\gamma^{2}+\left(b_{k}+h_{k} A\right)^{2}}{\gamma^{2}+\left(b_{k}-h_{k} A\right)^{2}}
\end{aligned}
$$

However, since (5.21) needs to be calculated every $k$, we propose a more simple approximation with lower complexity. For higher GSNR, the PWL-LLR parameters can be approximated as follows:

$$
\begin{aligned}
\tilde{a_{k}} & =h_{k} A \\
\tilde{c_{k}} & =2 \log \frac{2 h_{k} A}{\gamma} \\
\tilde{b_{k}} & =2 a_{k} \\
\tilde{d_{k}} & =0.2 c_{k}
\end{aligned}
$$

Approximating $d_{k}$ as a constant fraction of $c_{k}$ comes from Figure 5.12.

\subsubsection{Performance Comparison}

The performance of the PWL-LLR combiner with parameters in (5.21) and (5.22) is compared with that of the MRC, OLC, SC, $\mathrm{AO}$ and the optimal detectors when $\alpha=1.1,1.5$ and 1.8 and $N=4$ and 8 .

From Figures 5.23 and 5.24 We can see that the MRC combiner, which is the optimal for the Gaussian noise, behaves poorly compared to the optimal combiner. It has relatively better performance when $\alpha$ approaches 2 (the optimality point of the MRC), however it is still far from the optimal. The OLC, in most cases, has 


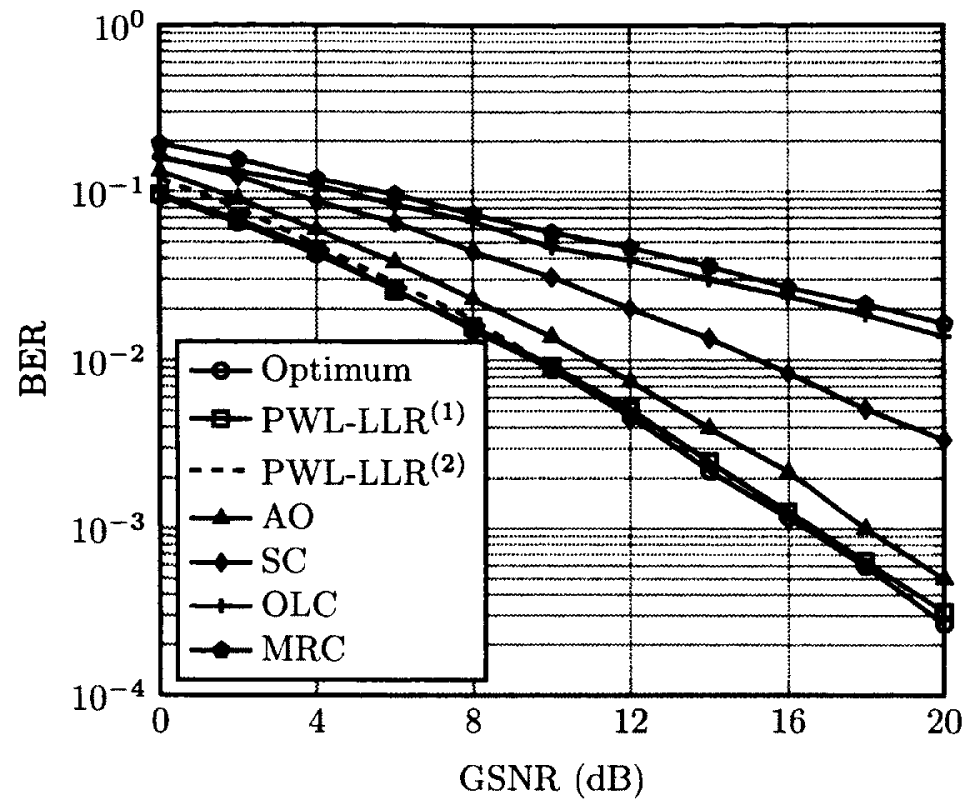

Figure 5.23: Performance comparison of the PWL-LLR combiner with $\alpha=1.1$, $N=4$.

performance almost equal to that of the MRC; nevertheless, it has slight improvement over the MRC when $\alpha$ approaches 1 (high impulsive noise) but it is still far from the optimal. The sign correlator gives better performance than the MRC when $\alpha$ approaches 1. When $\alpha$ increases, the SC needs higher GSNR to show improvement over the MRC. The performance difference between the SC and the MRC becomes larger with low values of $\alpha$ and $N$ due to the poor performance of the MRC and better performance of the $\mathrm{SC}$ in high impulsive noise. However, the $\mathrm{SC}$ has performance far from optimal.

The asymptotically optimal combiner $(\mathrm{AO})$ gives the best performance among the above mentioned combiners due to it ability to approximate the heavy-tail behavior of the $\mathrm{S} \alpha \mathrm{S}$ noise pdf. The test statistics of the AO combiner depends on a simplification of the asymptotic approximation of the decay rate of the pdf tail which results in a better approximation of the optimal test statistics than the mentioned suboptimal combiners. It can be seen that the AO combiner has a better performance when $\alpha$ 


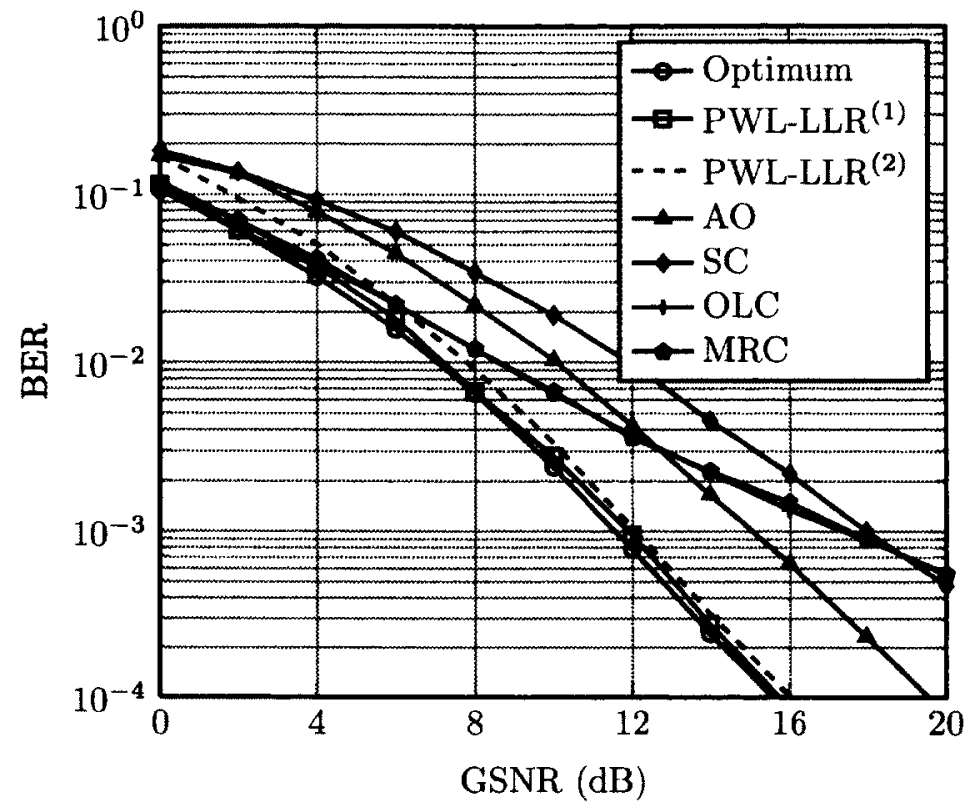

Figure 5.24: Performance comparison of the PWL-LLR combiner with $\alpha=1.8$, $N=4$.

approaches 1 and lower $N$ (around 1.7-2 dB off the optimal), however, it starts to degrade when $\alpha$ increases. 


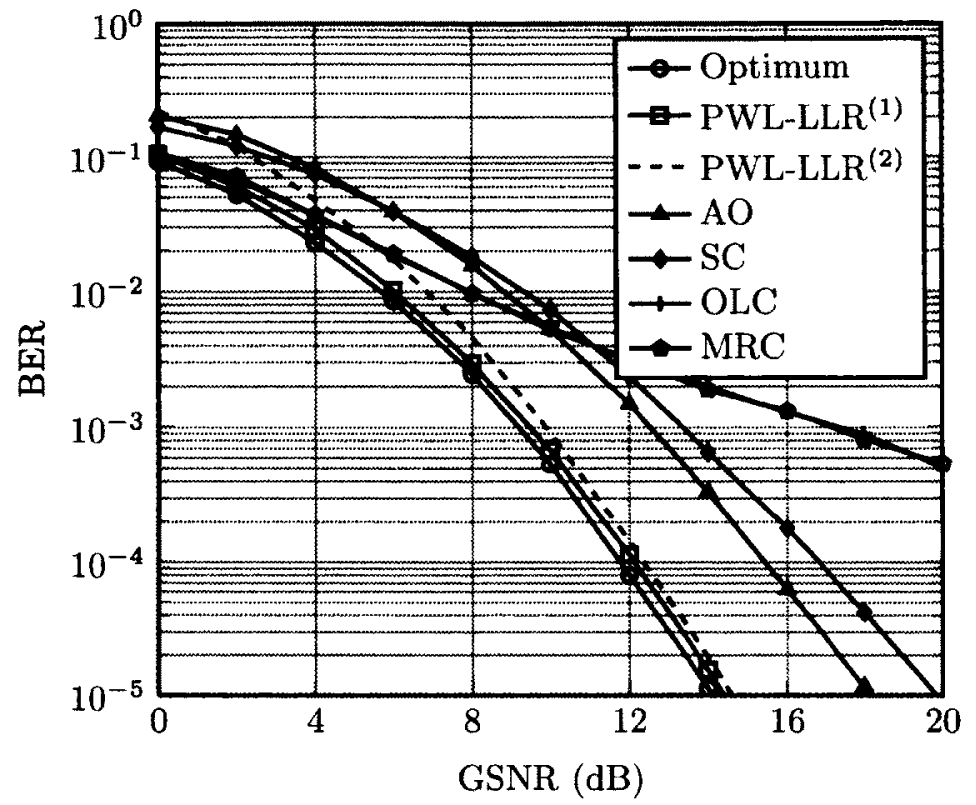

Figure 5.25: Performance comparison of the PWL-LLR combiner with $\alpha=1.8$, $N=8$.

Finally, the proposed PWL-LLR ${ }^{(1)}$ combiner with parameters in (5.21) shows robust superior near-optimal performance compared to the other suboptimal combiners. As shown in Figures 5.23, 5.24 and 5.25, the performance of the PWL-LLR combiner is closer to the optimal with low values of $\alpha$ and $N$. When $\alpha$ increases, its performance becomes around $0.1 \mathrm{~dB}$ off the optimal when $N=4$ and around $0.2 \mathrm{~dB}$ when $N=8$, as shown in Figure 5.25, which is much less than the degradation of the performance of the AO combiner. Also, it can be seen that the PWL-LLR ${ }^{(2)}$ combiner with the simplified parameters as in (5.22) gives near optimal performance with high GSNR, however, the performance degrades with low GSNR values and higher BER. This degradation is due to the high GSNR assumption which is used to approximate the PWL-LLR parameters in (5.22). For $N=4$, typically, the performance of the PWL-LLR ${ }^{(2)}$ is off the optimal by around $0.75 \mathrm{~dB}$ at $\mathrm{BER}=10^{-2}$ and $\alpha=1.1$ and is almost near the optimal at $\mathrm{BER}=10^{-5}$. The performance gap increases when $\alpha$ increases, but it keeps consistent performance by approaching the optimal when GSNR 
is higher and BER is around $10^{-5}$.

In summary, we can see that, near-optimal performance has been achieved by using the PWL-LLR combiner. By simplifying the calculations of the LLR parameters, the performance degrades with low GSNR and high BER regions for higher values of $\alpha$, yet it is still better than many other suboptimal combiners in this regions. Moreover, the performance of the proposed combiner with simplified parameters gives nearoptimal performance in the high GSNR and low BER region for different values of $\alpha$ and $N$.

\subsection{Conclusion}

In this section, we conclude this part of the thesis which contributes to the detection of signals in $\mathrm{S} \alpha \mathrm{S}$ noise. In this chapter, we proposed a simple yet generalized method based on the optimal log-likelihood ratios to design low-complexity suboptimal detectors with near-optimal performance for signals in $\mathrm{S} \alpha \mathrm{S}$ noise. The proposed method overcomes the limitations of the previous framework based on the optimal decision regions. As a result, we proposed the piecewise linear LLR (PWL-LLR) detector which simply approximates the optimal LLR using linear segments. The proposed PWL-LLR detector, which implicitly approximates the optimal decision boundaries, gives indistinguishable performance from the optimal detector with low complexity for all reasonable values of $\alpha$ and $N$. The PWL-LLR has been also used with the frequency-flat fading multipath channel as a simple suboptimal combiner. It gives superior near-optimal performance compared to different suboptimal combiners in the literature with low complexity. Moreover, a PWL-LLR combiner has been proposed with simplified parameters to save more on the calculations.

The proposed LLR-based method has been shown to be an effective tool to design low-complexity near-optimal detectors for signals in $\mathrm{S} \alpha \mathrm{S}$ noise. In the next chapter, 
we will show how to use the PWL-LLR approximation to design low-complexity decoders for coded signals in $\mathrm{S} \alpha \mathrm{S}$ noise with near-optimal performance. 


\section{Chapter 6}

\section{Log-likelihood Ratios: A Method to Design Suboptimal Decoders for Signals in $\mathbf{S} \alpha \mathbf{S}$ Noise}

\subsection{Introduction}

The optimal decoder for coded signals in $\mathrm{S} \alpha \mathrm{S}$ noise is impractical due to its complexity. A few attempts have been made to design simple suboptimal decoders, however, they depend on ad hoc approximations and they do not reach the optimal performance $[59,60]$. In this chapter, we will give a background and literature review of the decoder design for signals in SaS noise. Also, we will use the log-likelihood-based framework as a general approach to design simple suboptimal decoders for signals in SaS noise.

\subsection{Literature Review}

In this section, we will give a background and literature review of the decoder design for signals in $\mathrm{S} \alpha \mathrm{S}$ noise. The Viterbi and MAP decoding algorithms for convolutional codes in S $\alpha \mathrm{S}$ noise are discussed. The traditional Viterbi and MAP decoders, which 
have a branch metric optimized for Gaussian noise, perform poorly in symmetric $\alpha$ stable noise. Since the optimal maximum likelihood branch metric is impractically complex, many suboptimal decoders have been proposed in the literature, and these are described here.

\subsubsection{Viterbi Decoding in S $\alpha \mathrm{S}$ Noise}

The optimal maximum likelihood (ML) branch metric (BM) for the Viterbi decoder in $\mathrm{S} \alpha \mathrm{S}$ noise requires heavy computations due to the lack of a closed form expression for the probability density function, which requires an inverse Fourier transform to calculate [8]. Therefore, several suboptimal branch metrics have been proposed in the literature. The conventional Euclidean-norm (Gaussian) branch metric (Gauss-BM), which is optimal when $\alpha=2$ (the Gaussian case), is the least complex, however, it performs poorly when $\alpha$ is smaller.

Other simple suboptimal branch metrics use a $p$-norm nonlinearity which is considered a robust cost function in the presence of $\mathrm{S} \alpha \mathrm{S}$ noise [8]. For further simplification, the 1-norm $(p=1)$ branch metric, which is the absolute value, has been used in [59] as a low-complexity method to improve the performance of the Viterbi algorithm. Although this absolute branch metric (Abs-BM) gives better performance than the Gauss-BM, it is not robust against $\alpha$ as the performance becomes worse when $\alpha$ approaches 1. Also, the Abs-BM and other suboptimal metrics are heuristic approaches which have no adequate explanation for why they have such performance. Moreover, there is no clear methodology of designing a suboptimal Viterbi decoder other than ad-hoc methods.

\subsubsection{Optimal and Suboptimal Viterbi Decoders}

At time instant $i$, a group of $k_{c}$ message bits are applied to a rate $R_{c}=k_{c} / n_{c}$ convolutional encoder. The resulting $n_{c}$ code bits are mapped to BPSK symbols which 
are transmitted over the additive white $\mathrm{S} \alpha \mathrm{S}$ noise channel. The received symbols are

$$
r_{i, j}=c_{i, j}+n_{i, j}
$$

where $c_{i, j} \in\{ \pm A\}$ is the BPSK symbol corresponding to the $j^{\text {th }}$ code bit $(j \in$ $\left.\left\{1,2, \ldots, n_{c}\right\}\right)$ produced at time $i$, and $\left\{n_{i, j}\right\}$ are independent $S \alpha S$ noise samples. The average transmitted energy per message bit is $E_{b}=A^{2} / R_{c}$.

6.2.1.1.1 ML Viterbi Decoder The Viterbi algorithm can be used to perform the optimal maximum likelihood (ML) sequence decoding of the received samples. The ML branch metric (ML-BM) of the Viterbi algorithm at the $i^{\text {th }}$ time instant is:

$$
\mu_{M L}=-\sum_{j=1}^{n_{c}} \log f_{\alpha}\left(r_{i, j} \mid c_{i, j}\right)
$$

where $f_{\alpha}\left(r_{i, j} \mid c_{i, j}\right)$ is the conditional probability density function (pdf) of the received sample conditioned on the transmitted code bit. For simplicity, we will drop the time index, $i$, since the following analysis is applied to any time instant.

6.2.1.1.2 Gaussian Viterbi Decoder The ML branch metric requires complex computations to calculate the conditional pdf which makes the ML Viterbi algorithm impractical. As a suboptimal solution, the ML-BM is simplified assuming $\alpha=2$ in (6.2) (i.e., the noise is assumed to be Gaussian) [59]. The resultant branch metric is the Euclidean metric (Gauss-BM):

$$
\mu_{G a u s s}=\sum_{j=1}^{n_{c}}\left(r_{j}-c_{j}\right)^{2}
$$

However, the Viterbi decoder with the Gauss-BM gives poor performance compared to the optimal one when $\alpha<2$. 
6.2.1.1.3 Absolute-Branch Metric Viterbi Decoder To improve the decoder performance and maintain affordable complexity, the 1-norm branch metric was proposed in [59] as follows:

$$
\mu_{A b s}=\sum_{j=1}^{n_{c}}\left|r_{j}-c_{j}\right|
$$

Instead of using the Euclidean distance, the 1-norm branch metric uses the absolute value $^{1}$, and gives performance close to the Viterbi decoder with ML-BM when $\alpha$ is high $(\alpha>1.5)$. Also, the computational complexity of the Abs-BM is much lower than that of the ML-BM. However, the performance of the Abs-BM degrades when $\alpha$ gets smaller and approaches 1. Moreover, the use of the absolute value is based on a heuristic approach which is motivated by the robustness of the 1-norm nonlinearity in non-Gaussian noise $[61,62]$. There is no adequate explanation why the Abs-BM is better than the Gauss-BM and why it has near optimal performance for a certain range of $\alpha$ but has much worse performance in another range.

Also, we will use the Cauchy and the Hamming branch metrics to compare with the Abs-BM where Cauchy-BM is

$$
\mu_{\text {Cauchy }}=\sum_{j=1}^{n_{c}} \log \left(\gamma^{2}+\left(r_{j}-c_{j}\right)^{2}\right)
$$

and Hamming-BM is

$$
\mu_{\text {Hamming }, i}=\sum_{j=1}^{n_{c}} r_{j} \oplus c_{j}
$$

where $\oplus$ denotes the exclusive-OR.

\subsubsection{Performance Evaluation}

As a performance comparison, the probability of bit error $\left(P_{b}\right)$ of the Viterbi decoder with optimal and suboptimal branch metrics is evaluated by simulation for the case

\footnotetext{
${ }^{1}$ We will refer to the 1-norm branch metric as Abs-BM.
} 
when $\alpha=1$ and 1.7. Because the noise variance is not defined for values of $\alpha<2$, the geometric signal-to-noise ratio (GSNR), $\frac{1}{4 C_{g}}\left(\frac{E_{s}}{R_{c} S_{0}^{2}}\right)$, is used (as shown in (2.11)), where $E_{s}=A^{2}$ is the transmitted energy per symbol, and $S_{0}^{2}=C_{g}^{\frac{2}{\alpha}-2} \gamma^{\frac{2}{\alpha}}$ is the geometric power of the $\mathrm{S} \alpha \mathrm{S}$ random variable and $C_{g} \approx 1.78$ is the exponential of Euler's constant [49]. A 1/2 rate convolutional code is used with generator polynomials $(561)_{8}$ and $(753)_{8}$. To calculate an accurate $P_{b}$, up to $10^{6}$ frames with 400 bits each are transmitted, and the simulation is stopped when the number of bit errors reaches $10^{4}$ or $P_{b} \leq 10^{-5}$ is achieved.

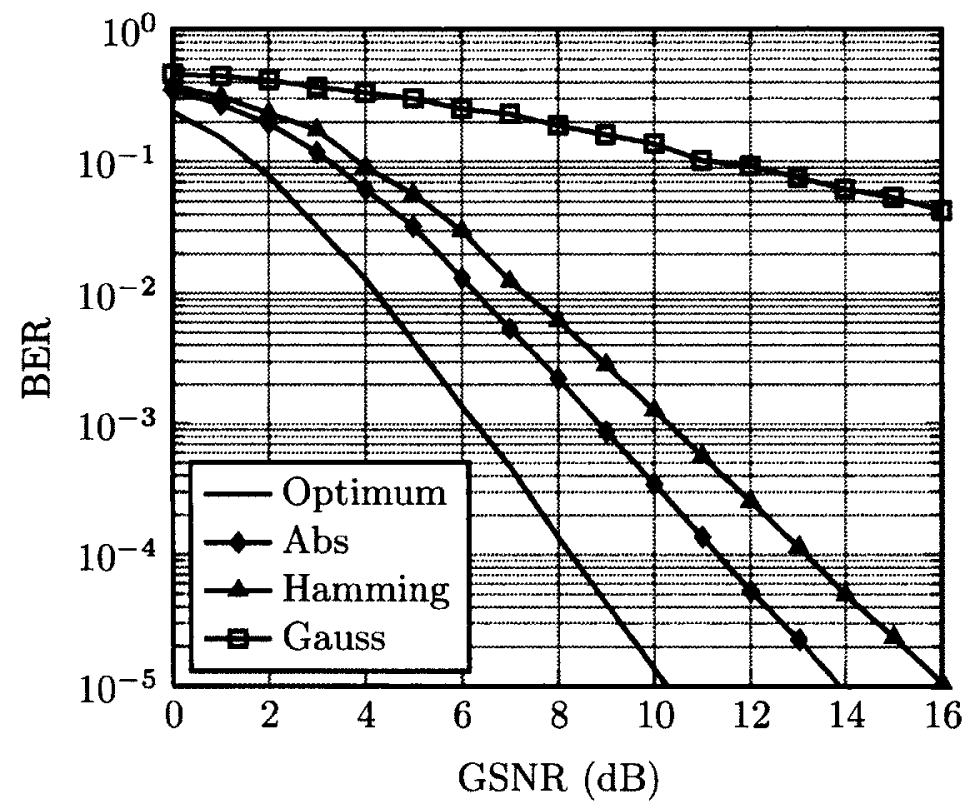

Figure 6.1: BER of the Viterbi decoder with different BMs with $\alpha=1$.

Figures 6.1 and 6.2 show the BER of different suboptimal Viterbi decoders in S $\alpha \mathrm{S}$ with $\alpha=1$ and $\alpha=1.7$. It can be shown that the Viterbi decoder with the conventional Gauss-BM gives poor performance compared to the ML-BM. Even the hard decision Viterbi decoder with the Hamming branch metric (Ham-BM) gives better performance than the Gauss-BM, due to the removal of the effect of the impulses by using the hard decision at the input of the decoder. However, the performance is still 
far from optimal.

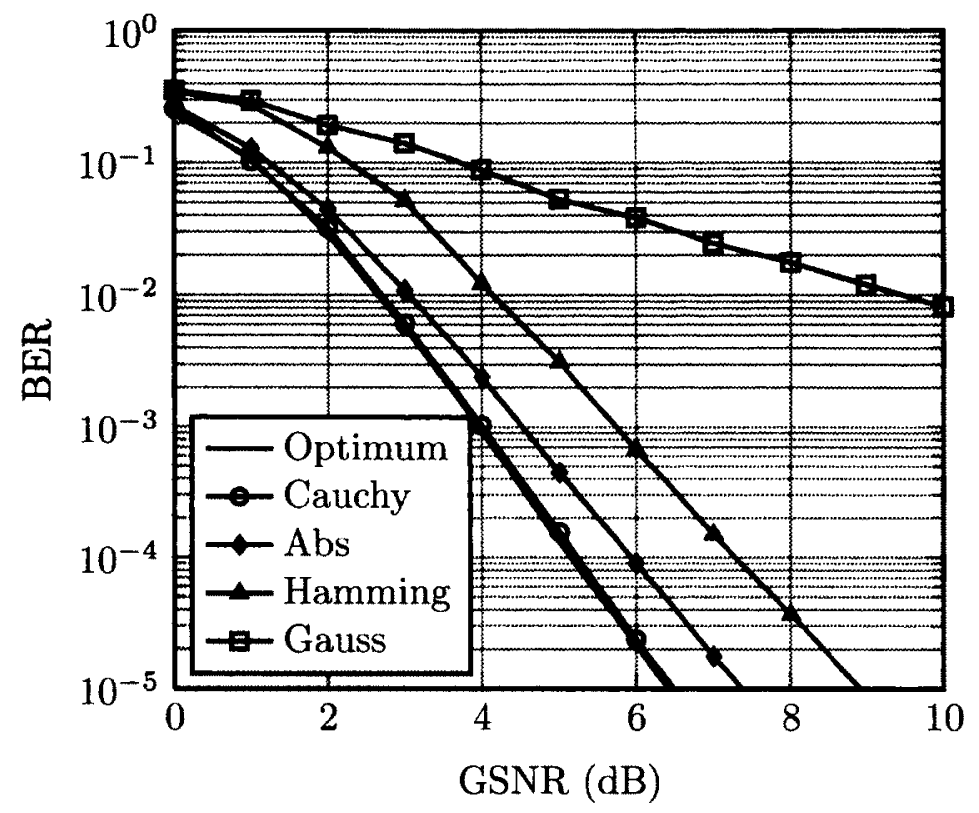

Figure 6.2: BER of the Viterbi decoder with different BMs with $\alpha=1.7$.

On the other hand, the Abs-BM gives better performance than the Gauss-BM and the Ham-BM (about $1 \mathrm{~dB}$ behind the performance using optimal ML-BM when $\alpha=1.7$ ), however, the performance is worse with lower values of $\alpha$ (around $4 \mathrm{~dB}$ behind the performance using ML-BM when $\alpha=1$ ). Although these results suggest that Abs-BM is a good suboptimal branch metric, particularly for high $\alpha$, there is no clear justification for why this is the case, or why Gauss-BM performs so poorly. In this thesis, we will show how to justify and explain the performance of different suboptimal branch metrics and also how to design low complexity suboptimal Viterbi decoders with near optimal performance.

\subsubsection{MAP Decoding in $\mathrm{S} \alpha \mathrm{S}$ Noise}

The MAP decoder is used to perform minimum bit error probability decoding of convolutional codes [63]. Also, it is used as a constituent soft output decoder in 
concatenated convolutional codes such as turbo codes. A considerable amount of research has been done on the design and implementation of the MAP decoder in Gaussian noise, however, there is little work related to the design of simple MAP decoders in $\mathrm{S} \alpha \mathrm{S}$ noise. In this section, we will give some background on different suboptimal MAP decoders for convolutional codes in SaS noise and evaluate their performance.

\subsubsection{Optimal and Suboptimal MAP Decoder}

To avoid problems with numerical instability, the MAP decoder is typically implemented in the log domain, using the log-MAP algorithm [64,65]. The optimal branch metrics are the same as for the Viterbi algorithm, except for a reversal of the sign, namely

$$
\lambda_{M L}=\sum_{j=1}^{n_{c}} \log f_{\alpha}\left(r_{i j} \mid c_{i j}\right) .
$$

For the case when $\alpha=2$, the Gaussian branch metrics

$$
\lambda_{\text {Gauss }}=-\frac{1}{2 \sigma^{2}} \sum_{j=1}^{n_{c}}\left(r_{j}-c_{j}\right)^{2}
$$

where $\sigma^{2}$ is the equivalent Gaussian noise variance, are optimal, but are suboptimal and perform poorly when $\alpha<2$. To improve the performance of the Gaussian logMAP decoder, a nonlinear preprocessor was used in [60] to filter the impulses in the received samples, however, the resultant performance is still far from optimal.

Another suboptimal solution is to use the branch metric with $\alpha=1$ (i.e., the noise is assumed to be Cauchy). The resultant branch metric is

$$
\lambda_{\text {Cauchy }}=-\sum_{j=1}^{n_{c}} \log \left(\gamma^{2}+\left(r_{j}-c_{j}\right)^{2}\right) .
$$

The log-MAP decoder with Cauchy branch metric shows near optimal performance 
for a wide range of $\alpha$, however, it still needs complex computations.

Another simple suboptimal technique uses the Huber nonlinearity, which is used as a robust cost function in the presence of impulsive noise [66], to design a simple suboptimal MAP decoder with branch metric $[67,68]$

$$
\lambda_{\text {Huber }}=-\frac{1}{2 \sigma^{2}} \sum_{j=1}^{n_{c}} \rho\left(r_{j}-c_{j}\right)
$$

where $\rho(x)$ is the Huber nonlinearity,

$$
\rho(x)= \begin{cases}\frac{1}{2} x^{2} & |x| \leq h \\ h|x|-\frac{1}{2} h^{2} & |x|>h\end{cases}
$$

where $h>0$ is a cutoff parameter to tune the robustness of the nonlinearity.

The Huber nonlinearity uses the least square estimator (which is equivalent to the Gaussian soft detector) for residuals smaller than $h$, however, it uses the least absolute or $L_{1}$ norm with the tails values. The log-MAP decoder with the Huber BM gives better performance than the Gaussian log-MAP decoder, however, it starts to get worse when $\alpha$ approaches 1 . Moreover, the use of the absolute value in the Huber $\mathrm{BM}$ is based on a heuristic approach which is motivated by the robustness of the 1-norm nonlinearity in non-Gaussian noise. Also, it does not consider the statistics of the $\mathrm{S} \alpha \mathrm{S}$ noise, instead, it uses only the heavy tail nature of the noise.

\subsubsection{Performance Evaluation}

As a performance comparison, the probability of bit error $\left(P_{b}\right)$ of the log-MAP decoder with optimal and suboptimal branch metrics is evaluated by simulation for the case when $\alpha=1$ and 1.7. Because the noise variance is not defined for values of $\alpha<2$, the geometric signal-to-noise ratio is used instead to define the SNR as $\frac{1}{4 C_{g}} \frac{E_{s}}{R_{c} S_{0}^{2}}$, 
where $E_{s}=A^{2}$ is the transmitted energy per symbol, $R_{c}$ is the code rate, and $S_{0}^{2}=C_{g}^{\frac{2}{\alpha}-2} \gamma^{\frac{2}{\alpha}}$ is the geometric power of the $\mathrm{S} \alpha \mathrm{S}$ random variable where $C_{g} \approx 1.78$ is the exponential of the Euler constant [49]. A $\frac{1}{2}$ rate recursive systematic convolutional code is used with generator polynomials $(53,75)_{8}$. When simulating the decoder with Huber nonlinearity, $h=3 \gamma$ is used [67]. To calculate an accurate $P_{e}$, up to $10^{6}$ frames with 400 message bits each are transmitted, and the simulation is stopped when the number of bit errors reaches $10^{4}$.

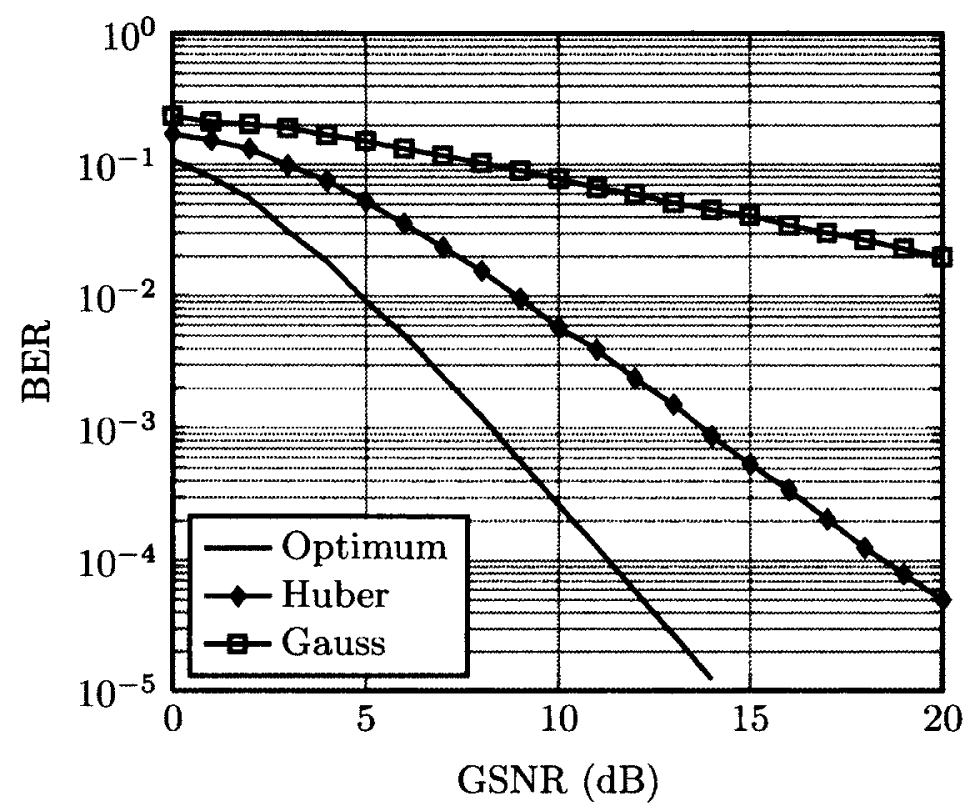

Figure 6.3: BER of the MAP decoder with different BMs with $\alpha=1$.

Figures 6.3 and 6.4 show that the BER of the MAP decoder with different branch metrics. The MAP decoder with the Gaussian BM gives poor performance compared to the MAP decoder with the optimal BM because of the heavy tail characteristic for noise with $\alpha<2$. On the other hand, the MAP decoder with Cauchy BM shows nearoptimal performance for different values of $\alpha$, however, there is no clear explanation why the Cauchy MAP decoder gives that performance. Also, its APPs need more computations than the Gaussian MAP decoder. 


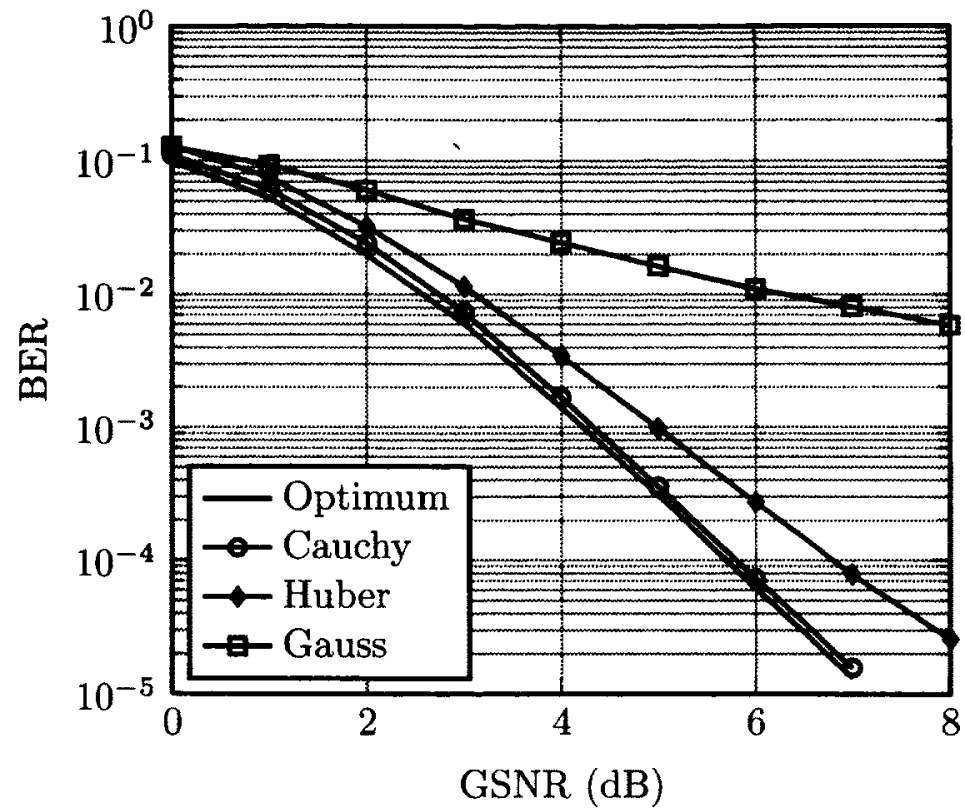

Figure 6.4: BER of the MAP decoder with different BMs with $\alpha=1.7$.

The MAP decoder with Huber BM, which uses a hybrid nonlinearity as in (6.11), gives better performance than the Gauss BM with affordable complexity especially with higher values of $\alpha$. For higher values of the received samples, the Huber nonlinearity uses the absolute norm to estimate the distance between the received and the transmitted code symbol which gives more robust estimation in non-Gaussian channels. However, the resultant performance still behind the optimal one especially when $\alpha$ approaches 1. Moreover, the use of the Huber nonlinearity does not depend on any optimal or suboptimal criteria to minimize the probability of bit error.

\subsubsection{Summary}

Since the optimal decoder is complex, many suboptimal solutions have bee presented in the literature. In this section, we presented a survey on different approaches to design low complexity decoders for coded signals in $\mathrm{S} \alpha \mathrm{S}$ noise. In particular, we considered the design of suboptimal Viterbi and MAP decoders. Most of the 
solutions starts from the Gaussian implementation of the decoder and try to adapt the decoder to accommodate the impulsive nature of the $\mathrm{S} \alpha \mathrm{S}$ noise. However, this adaptation does not meet a clear optimality or sub-optimality criteria to design the simplified decoder. For example, the absolute value metric has been used instead of the Euclidean metric (for Gaussian case) because the 1-norm nonlinearity shows robust performance as a cost function in non-Gaussian impulsive noise. Indeed, it gives better performance than the Gaussian decoder; however the performance is not robust against $\alpha$ and it is still behind the optimal. Moreover, it is difficult to use the same motivation behind the use of the 1-norm nonlinearity to improve the performance because it is a heuristic approach. Another example is the use of Huber nonlinearity instead of the Euclidean distance in case of the MAP decoder, which is also motivated by the robustness of the Huber nonlinearity as a cost function in the estimation of unknown parameters in non-Gaussian noise. The new nonlinearity gives also better performance than the Gaussian decoder but it is still behind the optimal. However, there is no clear explanation on why the performance improved and how we can extend on this approach to gain more improvement and robustness.

In the next section, we will show how to use the LLR-framework to justify and explain the performance of any suboptimal decoder and how to use the LLR as a unified metric to design simple suboptimal decoder. Furthermore, we will use the PWL-LLR proposed in Chapter 5 to design simple near-optimal decoders which follow clear approach to design and justify. 


\subsection{Design Near-Optimal Decoders using the LLR-Based Framework}

In this section, we will extend the use of the LLR-framework to design low-complexity suboptimal decoders with near-optimal performance by using simple design approach. It will be shown that the performance of different types of decoders depends mainly on the LLR of the received signal even if the decoding algorithm does not use the LLR explicitly as in the case of Viterbi decoder. As a result, we propose a simple design approach in which the suboptimal decoding algorithm should use, explicitly or implicitly, the closest simple approximation of the LLR. Therefore, the performance of the suboptimal decoder will be as close to the optimal performance as the LLR approximation to the optimal LLR. By using the proposed design approach with the Viterbi decoder, we propose a simple branch metric which improves the performance of the decoder compared to the Abs-BM-based Viterbi decoder. The proposed decoder uses an approximated LLR which is used implicitly in the branch metric. Moreover, by using the LLR explicitly in the Viterbi decoding algorithm, we are able to propose a simplified metric based on the PWL-LLR which closely approximates the optimal LLR. Therefore, the proposed PWL-LLR-based branch metric results in near-optimal performance with low complexity. The PWL-LLR branch metric has been also used with the MAP decoder which gives near-optimal performance compared to the MAP decoder with the Huber nonlinearity. The performance of the Viterbi and MAP decoders has been evaluated by simulation to verify the effectiveness of the proposed approach. 


\subsubsection{Proposed Suboptimal Viterbi Decoder}

In this section, we will show how to use the proposed approach with the Viterbi decoder to understand and justify the performance of the decoder with the AbsBM compared to the Gaussian one. Also, by using the LLR-based framework, we will

propose a simplified branch metric, DAbs-BM, which implicitly uses an approximated suboptimal LLR. So, we can gain performance improvement compared to the Abs-BM by using better approximation of the optimal LLR. Moreover, we will propose a new branch metric which explicitly uses the PWL-LLR to give near-optimal performance at low complexity.

\subsubsection{Performance Justification of the Optimal and Suboptimal Viterbi Algorithms using the LLR-Framework}

In general, for any two merging paths in the trellis diagram, the Viterbi algorithm selects as survivor the path that has the largest cumulative path metric. The cumulative path metric for hypothetical path $p$, up to and including the $k^{\text {th }}$ branch, is

$$
\beta_{k}(p)=-\sum_{i=1}^{k} \sum_{j=1}^{n_{c}} \log f_{\alpha}\left(r_{i, j} \mid c_{i, j}(p)\right)
$$

where $\left\{c_{i, j}(p)\right\}$ are the transmitted BPSK symbols for path $p$. If the correct path, $p_{c}$, merges with an incorrect path, $p_{x}$, at time $k$, the correct path will be pruned (i.e. a decoding error will occur) if $\beta_{k}\left(p_{x}\right)<\beta_{k}\left(p_{c}\right)$, which is equivalent to

$$
\beta_{k}\left(p_{x}\right)-\beta_{k}\left(p_{c}\right)=-\sum_{i=1}^{k} \sum_{j=1}^{n_{c}} \log \frac{f_{\alpha}\left(r_{i, j} \mid c_{i, j}\left(p_{x}\right)\right)}{f_{\alpha}\left(r_{i, j} \mid c_{i, j}\left(p_{c}\right)\right)}<0
$$

Because convolutional codes are linear, the probability of error does not depend on the transmitted codeword, so without loss of generality and to simplify the analysis, we can assume that the all-zero codeword is transmitted (i.e., $c_{i, j}\left(p_{c}\right)=-A \forall_{i, j}$ ). 
As such, the probability of error depends on the log likelihood ratios (LLR) of the received samples,

$$
\operatorname{LLR}_{O p t}(r)=\log \frac{f_{\alpha}(r \mid c=+A)}{f_{\alpha}(r \mid c=-A)}
$$

By comparing LLR $_{O p t}$ with the corresponding LLRs where the suboptimal branch metrics are used, it is possible to draw useful conclusions about the relative performance of the systems.

For the case when the Abs-BM is used, it can be shown that Abs-BM is a result of approximating the pdf of the S $\alpha \mathrm{S}$ noise by a Laplacian pdf:

$$
f(x)=D e^{-B|x|}
$$

where $\mathrm{D}$ and $\mathrm{B}$ are constant that depend on the noise variance and ensure unit area under the curve $f(x)$. The corresponding Laplacian LLR ${ }^{2}$ (Abs-LLR) can be calculated as follows:

$$
\operatorname{LLR}_{A b s}(r)=B[|r+A|-|r-A|]
$$

For the case when the Gaussian (Euclidean) branch metric (Gauss-BM), which results from approximating the $\mathrm{S} \alpha \mathrm{S}$ noise by a Gaussian pdf, the Gauss-LLR is:

$$
\operatorname{LLR}_{\text {Gauss }}(r)=C r
$$

where $C$ is a function of the noise variance. Note that the multiplicative scale factors in (6.16) and (6.17) have no effect on the Viterbi algorithm as they result in multiplicative factors that will cancel out in the decision rule in (6.13).

To explain the behavior of the Viterbi algorithm with the optimal and suboptimal

\footnotetext{
${ }^{2}$ We will refer to the Laplacian LLR by Abs-LLR to be consistent with the notation of the absolute branch metric Abs-BM.
} 


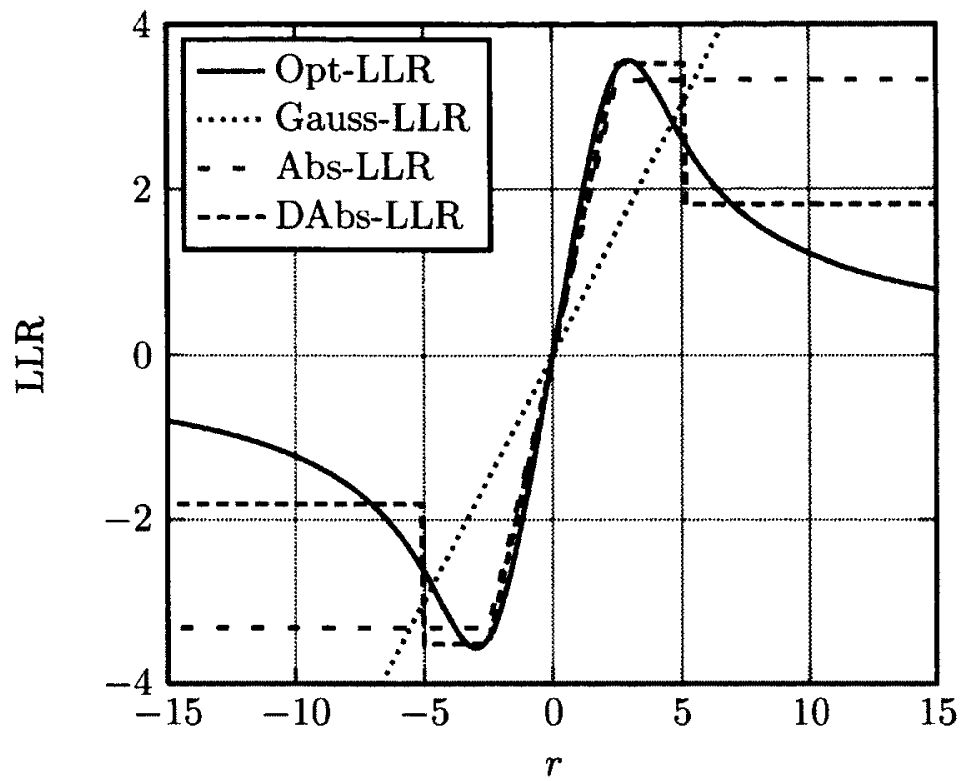

Figure 6.5: Optimal and suboptimal LLRs with $\alpha=1.3$ and GSNR $=5 \mathrm{~dB}$.

branch metrics, Figure 6.5 compares the Opt-LLR with Gauss-LLR, Abs-LLR and DAbs-LLR ${ }^{3}$. To give a fair comparison, the scale factors, $B$ and $C$, are optimized by minimizing the mean square error (MSE) of the fitting so that the Gauss-LLR and Abs-LLR have the best fit with the Opt-LLR. The MSE of the LLR fitting is

$$
\epsilon=\int_{-\infty}^{\infty}\left[L L R_{O p t}(r)-L L R_{s u b O p t}(r)\right]^{2} f(r) d r
$$

where $f(r)$ is the pdf of the received samples,

$$
f(r)=f(r \mid c=A) p(c=A)+f(r \mid c=-A) p(c=-A) .
$$

As can be seen in the figure, the Abs-LLR has a better fit to the Opt-LLR than the Gauss-LLR. Table 6.1 shows the normalized minimum MSE (MMSE) for the fitting of the Gauss-LLR and the Abs-LLR. The MMSE is evaluated numerically for

\footnotetext{
${ }^{3}$ The DAbs-LLR will be introduced in the next section.
} 
different values of $\alpha$ at $5 \mathrm{~dB}$. It can be seen that the Gauss-LLR has, in general, a significantly larger MMSE than the other LLRs, which explains the poor performance of the Viterbi decoder with Gauss-BM compared to the Opt-BM and Abs-BM, as shown in Section 6.2.1.2. On the other hand, the Abs-LLR has much lower MMSE than that of the Gauss-LLR. Also, as shown in the table, the MMSE of the Abs-LLR increases when $\alpha$ decreases which explains the performance degradation of the Viterbi decoder with the Abs-BM with smaller values of $\alpha$.

Table 6.1: Normalized MSE between the suboptimal and optimal LLR with GSNR $=5 \mathrm{~dB}$.

\begin{tabular}{|l|c|c|r|}
\hline$\alpha$ & Gauss-LLR & Abs-LLR & DAbs-LLR \\
\hline 1.1 & 0.5521 & 0.0646 & 0.0165 \\
1.2 & 0.5480 & 0.0583 & 0.0154 \\
1.3 & 0.5412 & 0.0527 & 0.0146 \\
1.4 & 0.5296 & 0.0470 & 0.0142 \\
1.5 & 0.5120 & 0.0426 & 0.0139 \\
1.6 & 0.4903 & 0.0375 & 0.0135 \\
1.7 & 0.4616 & 0.0324 & 0.0131 \\
1.8 & 0.4253 & 0.0263 & 0.0121 \\
1.9 & 0.3760 & 0.0190 & 0.0107 \\
\hline
\end{tabular}

\subsubsection{The Dual Absolute Branch Metric: Implicit Use of LLR}

In the previous section, we started by the branch metric to understand how implicitly it uses an approximate version of the LLR. In this section, we will start by introducing a better fit of the LLR, then, the corresponding branch metrics will be calculated which reflect the LLR approximation. The resultant branch metric shows performance improvement over the Abs-BM especially when $\alpha$ approaches 1 . 
It can be seen in Figure 6.5 that the Abs-LLR has a good fit with the Opt-LLR when the received samples are small, however, it deviates from the optimal for larger values of $r$, which have a non-negligible probability of occurrence due to the heavy tail of the $\mathrm{S} \alpha \mathrm{S}$ noise. To improve the performance and while keeping the branch metric simple, we propose the use of a dual absolute LLR (DAbs-LLR), also shown in Figure 6.5, which uses a flat line to better fit the tail portion of the optimal LLR. The proposed LLR can be considered as a result of using two different Laplacian pdfs to fit the center and tail regions of the $\mathrm{S} \alpha \mathrm{S}$ pdf, so, the proposed LLR is called a dual absolute LLR (DAbs-LLR) ${ }^{4}$. It is given by

$$
\operatorname{LLR}_{D A b s}(r)= \begin{cases}B_{1}(|r+A|-|r-A|) & |r| \leq \rho \\ B_{2}(|r+\rho|-|r-\rho|) & |r|>\rho\end{cases}
$$

where $B_{1}$ and $B_{2}$ are scale factors that are optimized to achieve the MMSE fit to the optimum LLR in each region and $\rho$ is a threshold value defining the boundary between the regions.

The proposed dual absolute LLR results in a new branch metric which is called a dual Abs-BM (DAbs-BM) as follows:

$$
\mu_{D A b s}=\sum_{j=1}^{n_{c}} \mu\left(r_{j} \mid c_{j}\right)
$$

where

$$
\mu\left(r_{j} \mid c_{j}\right)= \begin{cases}B_{1}\left|r_{j}-c_{j}\right| & \left|r_{j}\right| \leq \rho \\ B_{2}\left|r_{j}-\rho \frac{c_{j}}{A}\right| & \left|r_{j}\right|>\rho\end{cases}
$$

The proposed DAbs-BM is simply two different Abs-BMs scaled and shifted differently. The optimal scale and shift values, $B_{1}, B_{2}$ and $\rho$, are found by minimizing the MSE. It is mathematically difficult to minimize the MSE because it requires the

\footnotetext{
${ }^{4}$ To be consistent with the Abs-LLR.
} 
computation of the pdf of the $\mathrm{S} \alpha \mathrm{S}$ noise, which has no closed form. We propose to use a simple yet efficient approximation of the threshold $\rho$ to be $2 A$, where $A$ is the amplitude of the transmitted BPSK symbol. So, we allow the first piece of the DAbsLLR to span a range of $-2 A$ to $2 A$ which is a good approximation that captures the low and moderate values of the received samples, as shown in Figure 6.5. Then, the second piece of the Dual-LLR deals with the impulses in the received samples. For further simplification, it can be shown that the performance of the Viterbi algorithm depends only on the ratio $B_{2} / B_{1}$ if we take $B_{1}$ as a scale factor out of the branch metric in (6.21). Figure 6.6 shows the ratio $B_{2} / B_{1}$ that minimizes the MSE, versus the GSNR, for different values of $\alpha$. As an approximation, we use a single linear fit for all values of $\alpha$ to calculate the ratio $B_{2} / B_{1}$ as a function of the GSNR.

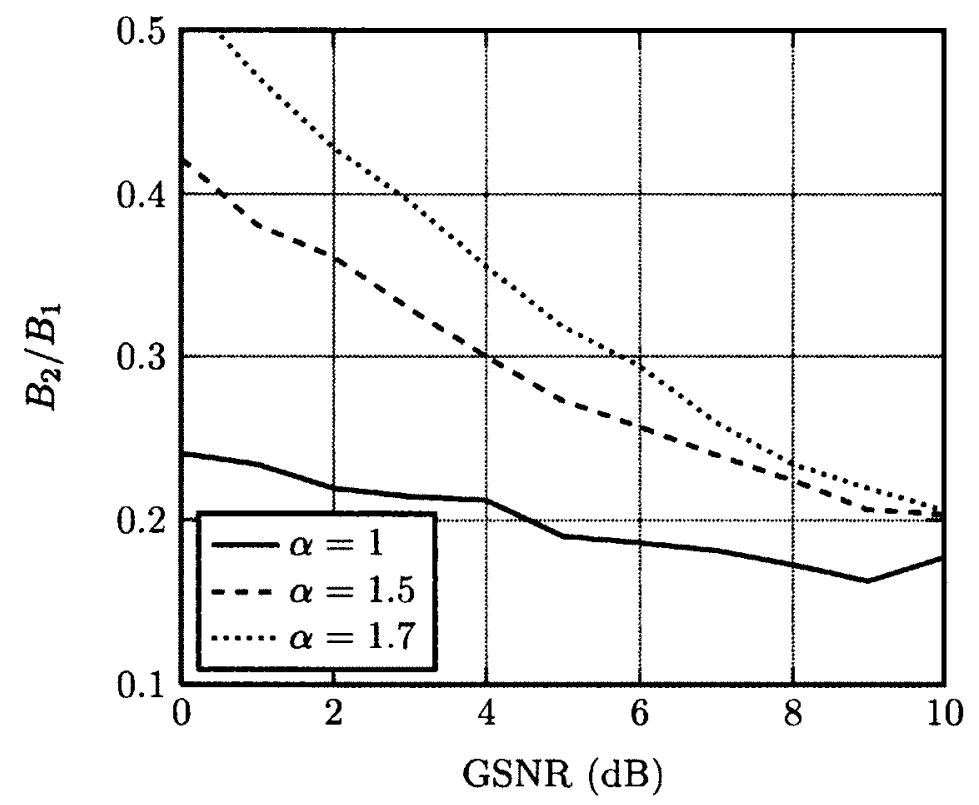

Figure 6.6: The ratio of the optimal scale factors $B_{2} / B_{1}$.

\subsection{Performance Evaluation and Simulation Results The perfor-} mance of the Viterbi decoder with the proposed dual absolute branch metric (DAbs$\mathrm{BM}$ ) is evaluated by simulation with the same setup as in Section 6.2.1.2. 


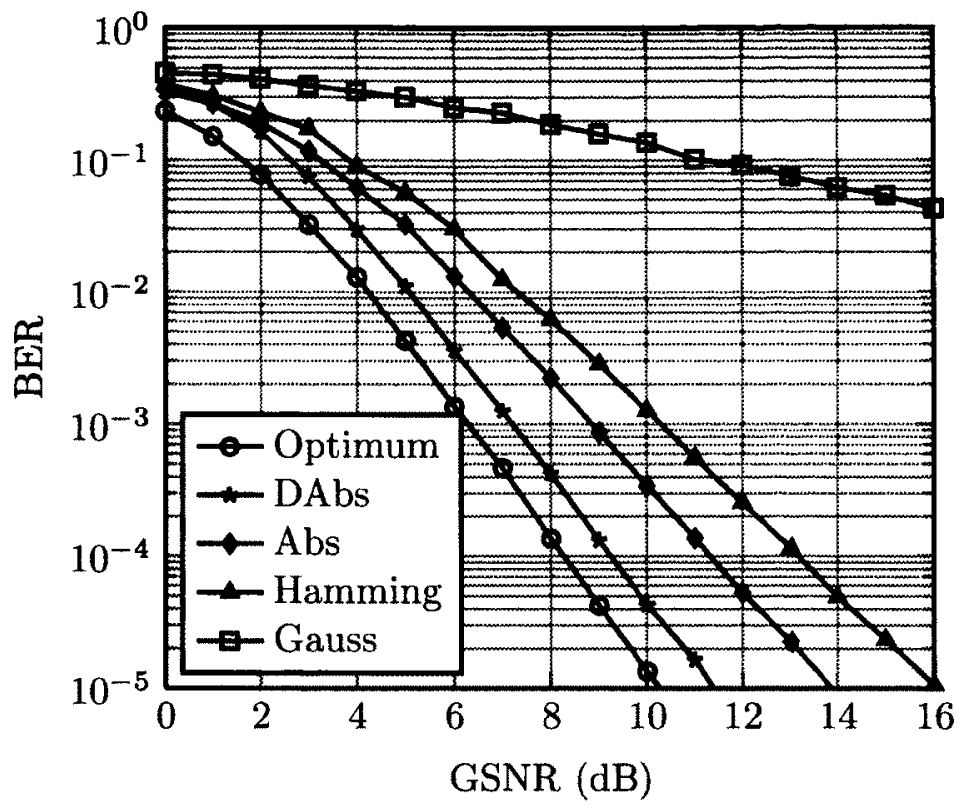

Figure 6.7: BER of the Viterbi decoder with DAbs BM and $\alpha=1$.

The performance of the DAbs-BM is evaluated using the approximate threshold, $\rho=2 A$ and the approximate scale factor ratio $B_{2} / B_{1}$ that linearly fits the optimal one shown in Figure 6.6. Figures 6.7 to 6.9 show the BER of the Viterbi decoder with the proposed branch metric compared to the Abs-BM and Gauss-BM proposed in [59] for different values of $\alpha$. 


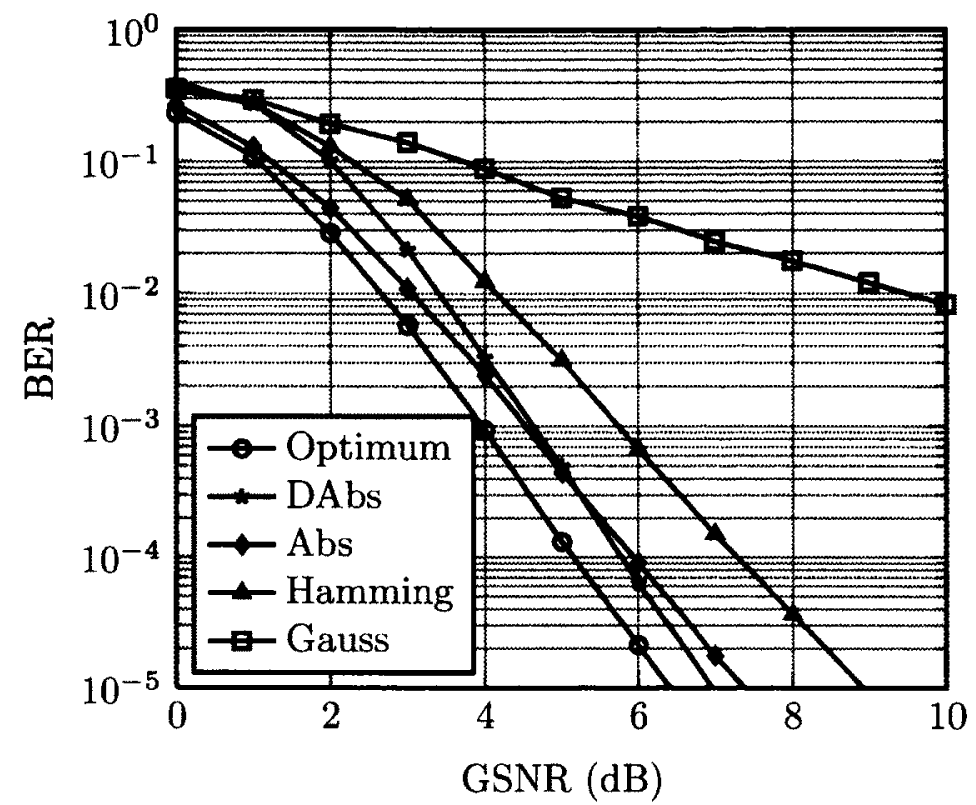

Figure 6.8: BER of the Viterbi decoder with DAbs BM and $\alpha=1.5$.

It can be seen that the performance of the DAbs-BM has a gain over the performance of the Abs-BM for different values of $\alpha$, especially when $\alpha=1$ where it has a gain of $2 \mathrm{~dB}$ over the Abs-BM as shown in Figure 6.7. This performance gain is expected because the DAbs-LLR has a better fit to the optimum LLR than that of the Abs-BM. As shown in Table 6.1, the MMSE of the DAbs-LLR is, in general, lower than the Abs-LLR especially for lower values of $\alpha$. However, it still increases when $\alpha$ decreases. So, this relatively low MMSE explains the performance gain of the Viterbi decoder with DAbs-BM over the Abs-BM for low values of $\alpha$. However, the performance of the DAbs-BM degrades when $\alpha$ increases as shown in Figure 6.9 where $\alpha=1.7$. 


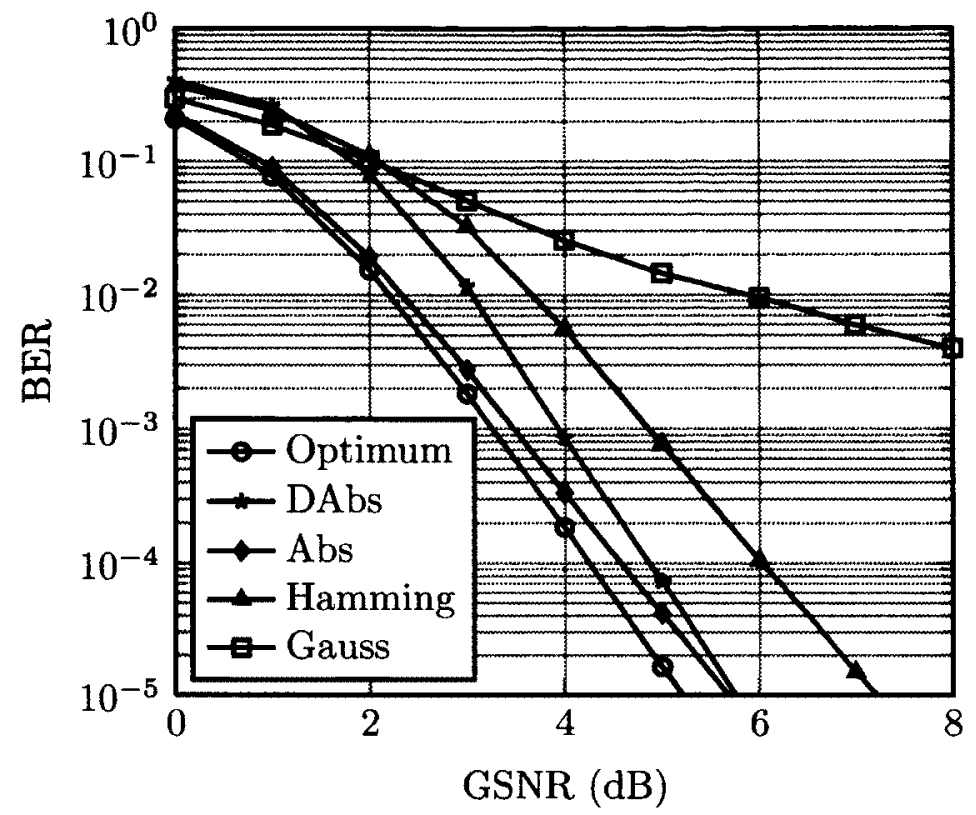

Figure 6.9: BER of the Viterbi decoder with DAbs BM and $\alpha=1.7$.

\subsubsection{The PWL-LLR Branch Metric}

Now, we have shown that the performance of the Viterbi algorithm depends on the LLR of the received symbols. So, we proposed to use the PWL-LLR approximation, which has been introduced in Chapter 5, to approximate the optimal LLR. Also, we rewrite the branch metric as a function of the LLR, therefore, the proposed branch metric needs simple calculation as it depends on linear segments only.

To rewrite the ML branch metric as a function of the LLR, we consider the following: at a given state, for any two merging paths, $P_{1}$ and $P_{2}$, at time instant $k$ with cumulative path metrics $\beta_{1}^{(k-1)}$ and $\beta_{2}^{(k-1)}$, respectively, we assuming that the survivor path is $P_{1}$ with cumulative metric $\beta_{1}^{(k-1)}$, so the following decision should hold

$$
\beta_{1}^{(k-1)}+\mu_{1}>\beta_{2}^{(k-1)}+\mu_{2}
$$


By substituting $\mu_{i}$ by $(6.2)$,

$$
\beta_{1}^{(k-1)}-\sum_{j=1}^{n_{c}} \log f_{\alpha}\left(r_{k, j} \mid c_{k, j}^{\beta_{1}}\right)>\beta_{2}^{(k-1)}-\sum_{j=1}^{n_{c}} \log f_{\alpha}\left(r_{k, j} \mid c_{k, j}^{\beta_{2}}\right)
$$

where $c_{k, j}^{\beta_{1}}$ and $c_{k, j}^{\beta_{2}}$ are the code symbols of the trellis branches emerging from path $P_{1}$ and $P_{2}$ at time $k$, receptively.

By adding the constant $\sum_{j=1}^{n_{c}} \log f_{\alpha}\left(r_{k, j} \mid c_{k, j}=-1\right)$ to both sides, the decision rule becomes

$$
\beta_{1}^{(k-1)}+\mu_{\mathrm{LLR}}^{(1)}>\beta_{2}^{(k-1)}+\mu_{\mathrm{LLR}}^{(2)}
$$

where

$$
\mu_{L L R}=-\sum_{j=1 \mid c_{j}^{0}= \pm 1}^{n_{c}} \operatorname{LLR}\left(r_{j}\right)
$$

and the superscript 1,2 indicates the branch corresponding to the path $P_{1}$ and $P_{2}$, respectively, and the summation is over all values of $j$ with a code bit value of $c_{j}=1$ in the corresponding branch. Therefore, by using 6.26 , we are able to calculate the branch metric as a function of the LLR only. At this point, we propose to use the simple piecewise linear approximation of the optimal LLR, PWL-LLR, to calculate the branch metric used in the Viterbi algorithm. The resulting branch metric is

$$
\mu_{L L R}=-\sum_{j=1 \mid c_{j}^{0}= \pm 1}^{n_{c}} \operatorname{PWL-LLR}\left(r_{j}\right)
$$

where the PWL-LLR is introduced in (5.6). The proposed Viterbi algorithm with PWL-LLR-based branch metric is shown to give near optimal performance with less computation complexity compared to the optimal one.

6.3.1.3.1 Performance Evaluation and Simulation Results The performance of the Viterbi decoder with the PWL-LLR is evaluated by simulation with 
the same setup as in Section 6.2.1.2.

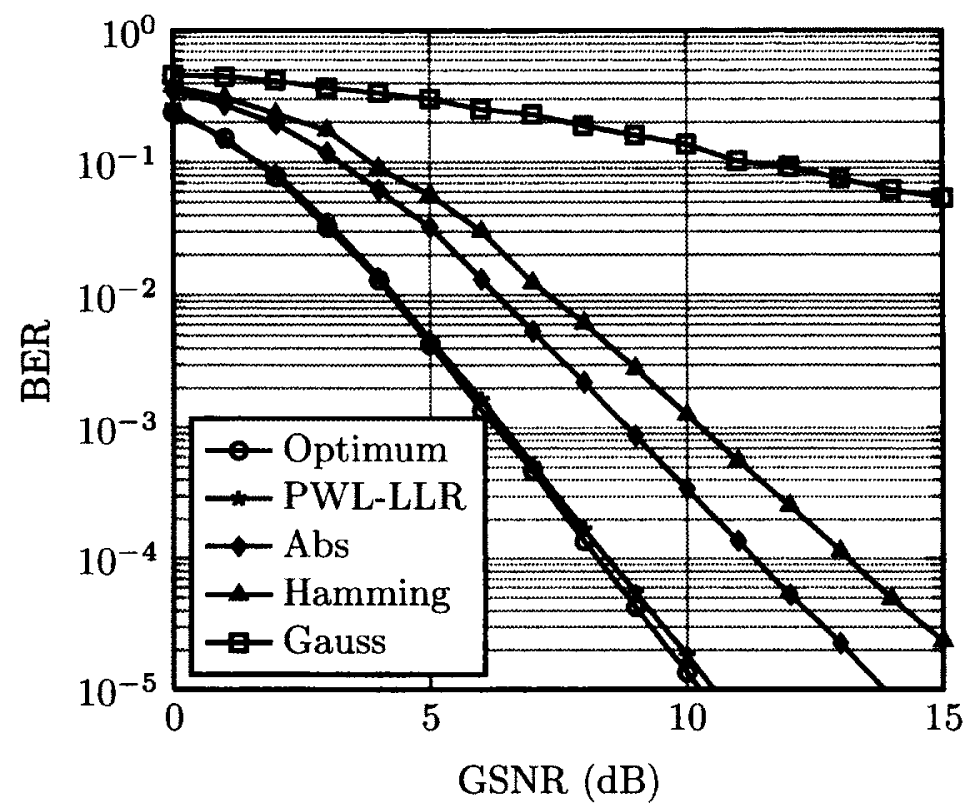

Figure 6.10: BER of the Viterbi decoder with PWL-LLR and $\alpha=1$.

The performance of the proposed PWL-LLR Viterbi decoder is compared with that of the Viterbi decoder with DAbs-BM and Abs-BM. Figures 6.10, 6.11, and 6.12 show the BER of the Viterbi decoder with different values of $\alpha$. It can be shown that the Viterbi decoder with PWL-LLR BM gives near optimal performance compared to the Abs-BM especially for values of $\alpha$ approaching 1 . The reason for that performance difference is expected because the PWL-LLR branch metric is based on a more accurate approximation of the optimal LLR. Also both the PWL-LLR-BM and the DAbs-BM have comparable computation complexity. However the PWLLLR-BM depends on four fitting parameters, $a, b, c$ and $d$, as shown in Section 5.2. 


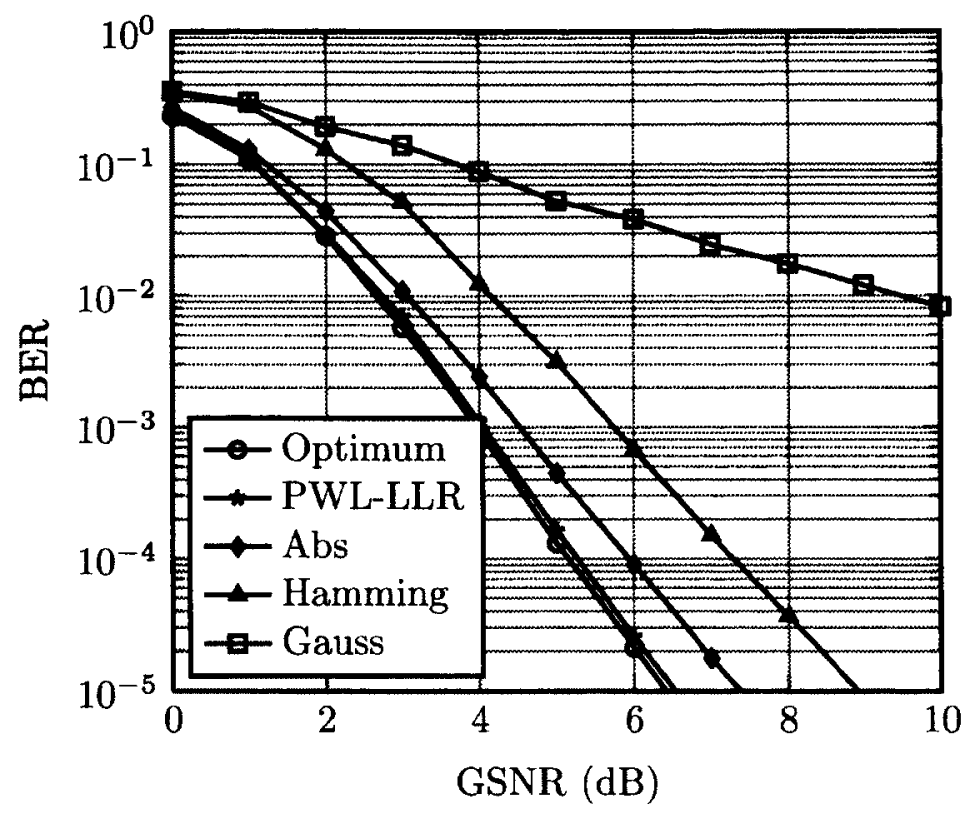

Figure 6.11: BER of the Viterbi decoder with PWL-LLR and $\alpha=1.5$.

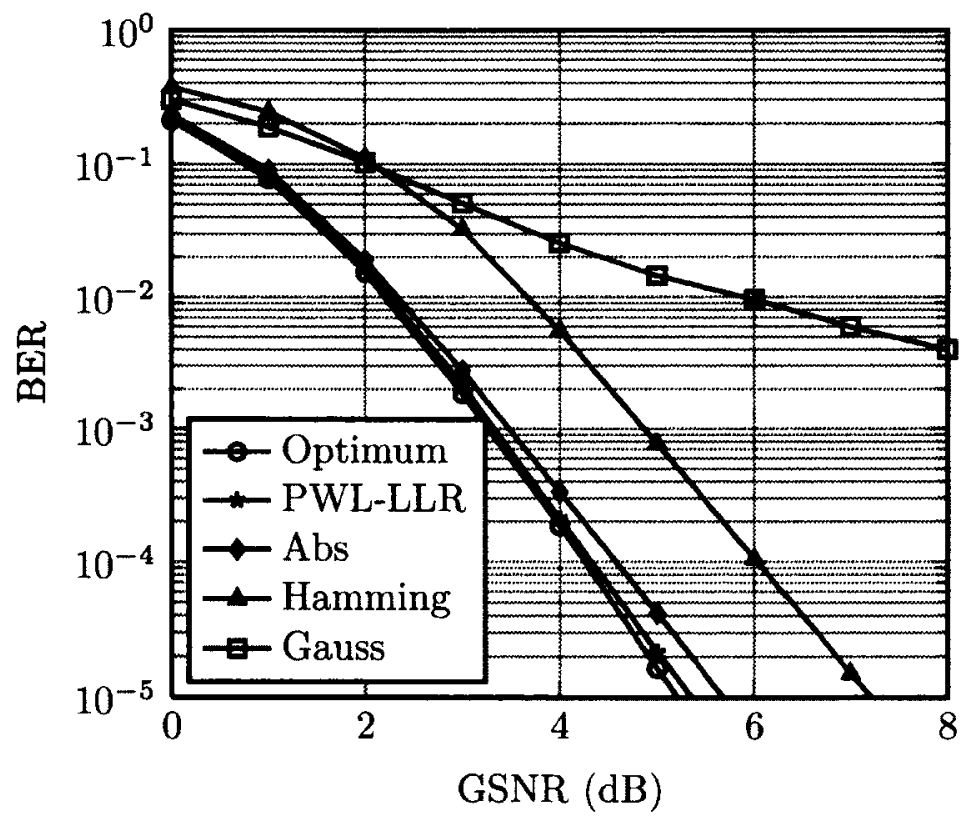

Figure 6.12: BER of the Viterbi decoder with PWL-LLR and $\alpha=1.7$. 


\subsubsection{Proposed Suboptimal MAP Decoder}

As shown in Section 6.2.2, there are several suboptimal MAP decoders with different computation complexity and performance. The least complex one is the Gaussian MAP decoder with poor performance. The Cauchy MAP decoder gives near-optimal performance with more computation complexity. The MAP decoder with the Huber nonlinearity gives better performance than the conventional MAP decoder with Gauss $\mathrm{BM}$, and also, it has less computation complexity than that of the MAP decoder with Cauchy BM. In this section, we will use the LLR-based framework to design low-complexity MAP decoders by using the PWL-LLR approximation explicitly in the MAP algorithm.

\subsubsection{Log-MAP Decoder Based on PWL-LLR}

To avoid numerical instability, we will consider the design of the log-MAP algorithm which performs its calculations based on the channel LLR for each received sample, $r_{i j}$, given by

$$
L L R_{O p t}\left(r_{i j}\right)=\log \frac{f_{\alpha}\left(r_{i j} \mid c_{i j}=+A\right)}{f_{\alpha}\left(r_{i j} \mid c_{i j}=-A\right)}
$$

where $L L R_{o p t}\left(r_{i j}\right)$ is the optimal LLR which depends on the likelihood function $f_{\alpha}\left(r_{i j} \mid c_{i j}= \pm A\right)$ which is the conditional probability density function $r_{i j}$ given $c_{i j}$. It can be shown that the Gaussian MAP algorithm uses

$$
L L R_{G a u s s}\left(r_{i j}\right)=\frac{2 A}{\sigma^{2}} r_{i j}
$$

where $\sigma^{2}$ is the equivalent Gaussian noise variance. Similarly, the Cauchy MAP algorithm uses

$$
L L R_{C a u c h y}\left(r_{i j} \mid c_{i j}\right)=\log \frac{\gamma^{2}+\left(r_{i j}+A\right)^{2}}{\gamma^{2}+\left(r_{i j}-A\right)^{2}}
$$


and the MAP algorithm with the Huber nonlinearity uses

$$
L L R_{H u b e r}\left(r_{i j}\right)=\left\{\begin{array}{lc}
\frac{2 A}{\sigma^{2}} r_{i j} & \left|r_{i j}\right| \leq h-A \\
\frac{\operatorname{sgn}\left(r_{i j}\right)}{\sigma^{2}}\left[h\left(\left|r_{i j}\right|+A\right)-\frac{1}{2} h^{2}-\frac{1}{2}\left(\left|r_{i j}\right|-A\right)^{2}\right] & \\
& h-A<\left|r_{i j}\right| \leq h+A \\
\operatorname{sgn}\left(r_{i j}\right) \frac{2 A h}{\sigma^{2}} & \left|r_{i j}\right|>h+A
\end{array}\right.
$$

By comparing the optimal and suboptimal LLRs, it is possible to draw useful conclusions about the relative performance of the log-MAP decoder. To explain the behavior of the MAP algorithm with the optimal and suboptimal LLRs, Figure 6.13 shows the optimal LLR for a given value of $\alpha$ and compares it to the Cauchy, Huber and Gaussian LLRs. As can be shown from the figure, the Gaussian LLR gives a good approximation to the optimal LLR when $r$ is small, however, it over-estimates the optimal LLR for higher values of $r$ which have a relatively high probability of occurrence in a heavy tail noise like $\mathrm{S} \alpha \mathrm{S}$ noise. This inaccurate fit explains the poor performance of the MAP decoder with Gaussian LLR. The Huber LLR gives a better, yet still inaccurate, fit of the tail region of the LLR due to the use of the absolute norm in that region which results in approximating the optimal LLR with a constant line. This approximate fit explains the performance gain of using the Huber LLR compared to the Gaussian LLR. Finally, it can be shown that the LLR with $\alpha=1$, which is the Cauchy LLR, has a close fit to the optimal LLR with different values of $\alpha$, which explains the near-optimal performance of the MAP decoder with Cauchy LLR. 


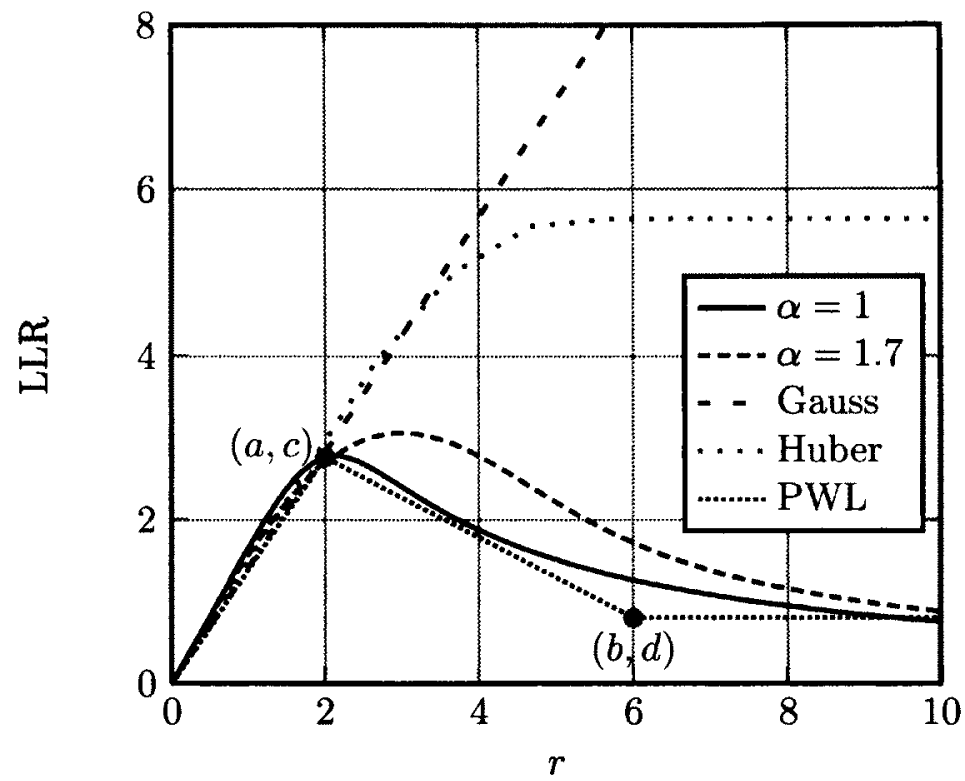

Figure 6.13: Comparison between the optimal and different suboptimal LLRs.

Motivated by the effect of the dependency of the MAP algorithm on the LLR only, we propose to use the PWL-LLR, which is a simple piecewise linear approximation of the optimal LLR proposed in Chapter 5, with the MAP algorithm to get better performance with low complexity.

The fitting parameters of the PWL-LLR in (5.6) can be simply calculated on-line for different GSNR values or even calculated off-line and stored in a look-up table. As such, the computation complexity of the MAP algorithm using the PWL-LLR has less complexity than the optimal and the Cauchy LLRs.

\subsubsection{Performance Evaluation}

The performance of the MAP decoder with the proposed PWL-LLR is evaluated by simulation with the same setup as in Section 6.2.2.2. The MAP decoder is implemented in the log domain by using the log-MAP algorithm. 


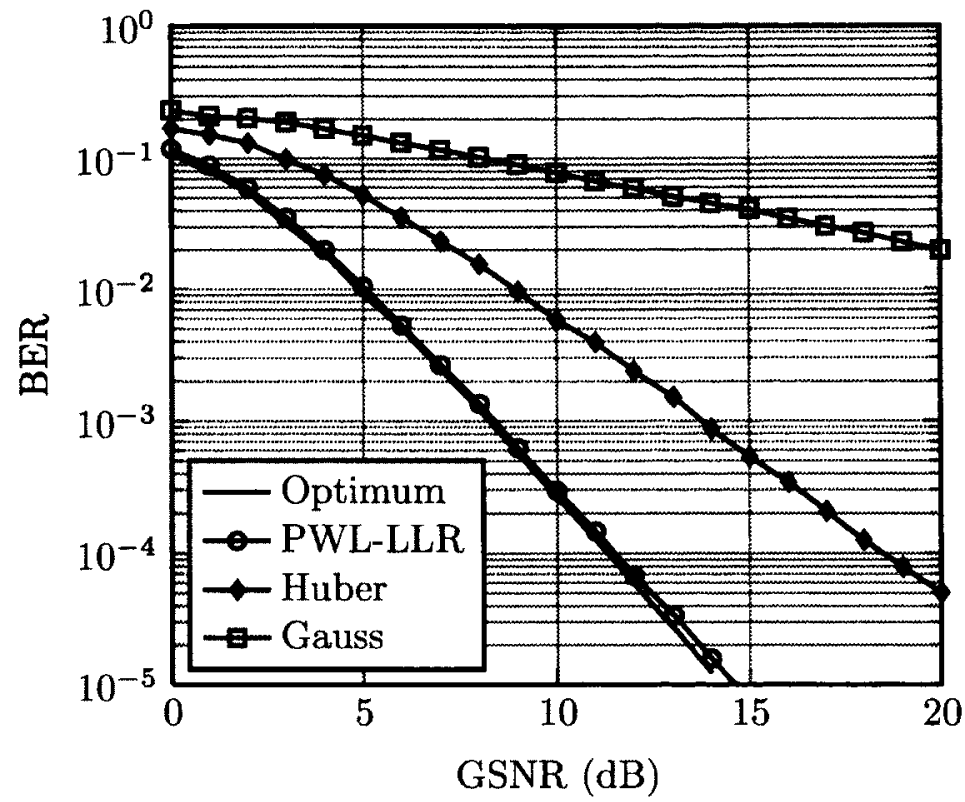

Figure 6.14: BER of the MAP decoder with PWL-LLR and $\alpha=1$.

Figures $6.14,6.15$, and 6.16 show the performance of the MAP decoder with the proposed PWL-LLR compared to the optimal, Gaussian and Huber LLRs for different values of $\alpha$. It can be shown that the MAP decoder with PWL LLR has near-optimal performance for different values of $\alpha$, which is around $2 \mathrm{~dB}$ (when $\alpha=1.5$ ) and $6 \mathrm{~dB}$ (when $\alpha=1$ ) better than the performance with the Huber LLR. This performance gain is due to the closer fit the PWL-LLR has compared to the other suboptimal LLRs. 


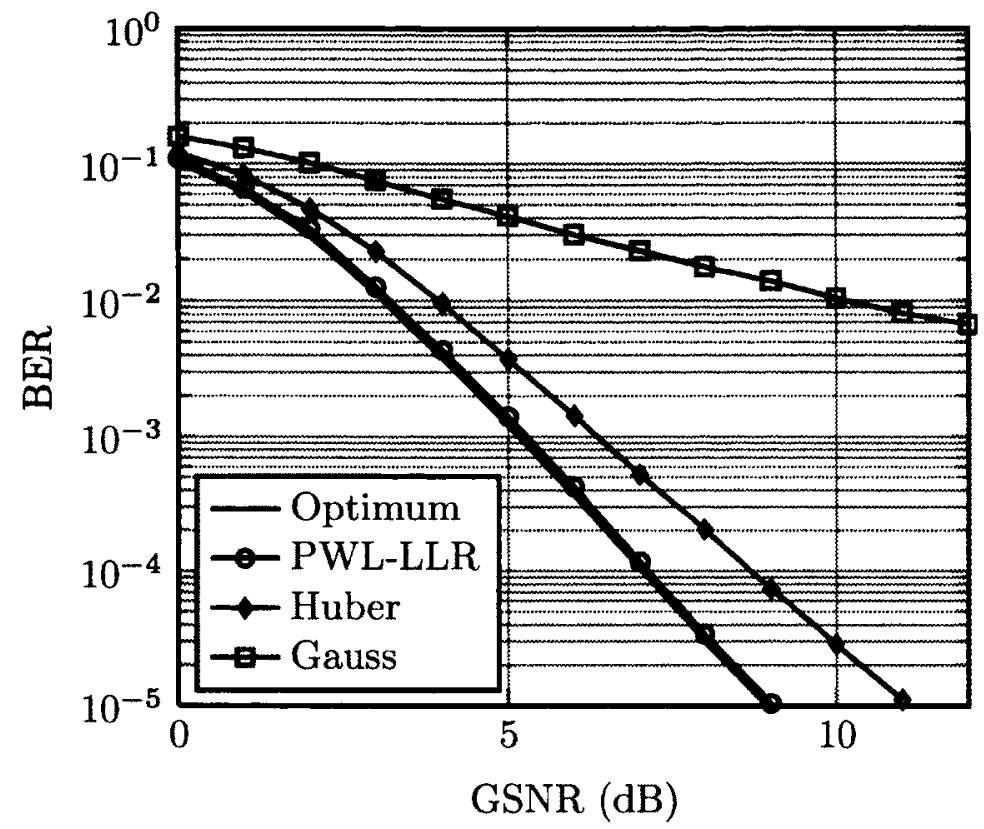

Figure 6.15: BER of the MAP decoder with PWL-LLR and $\alpha=1.5$.

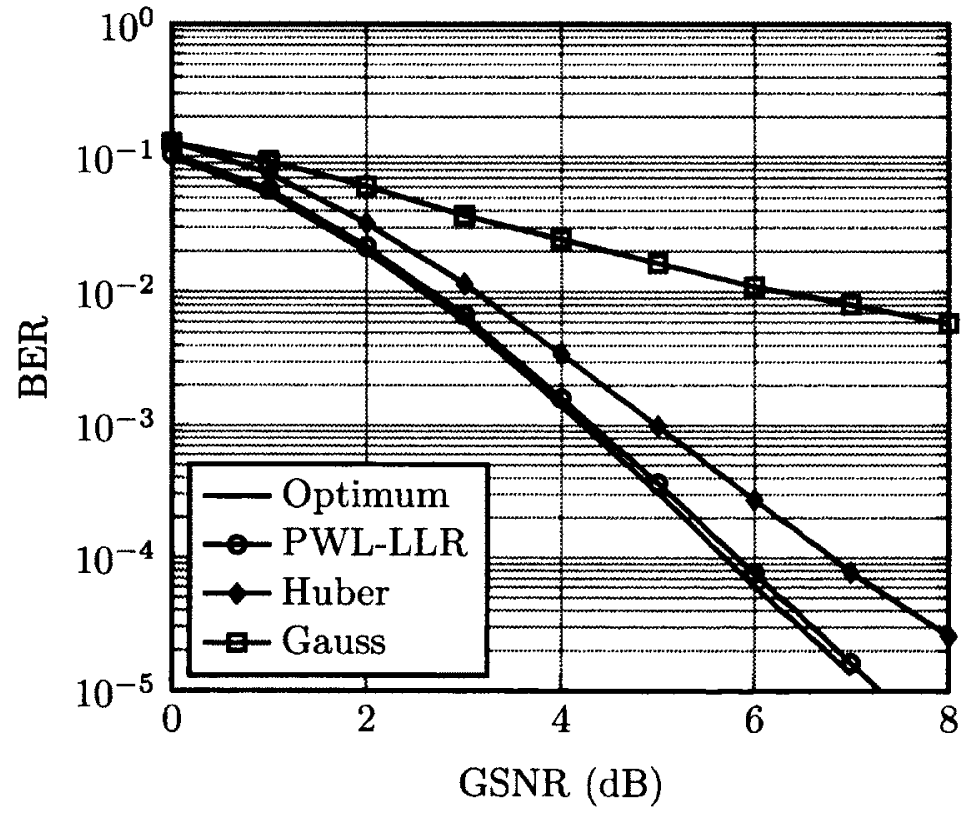

Figure 6.16: BER of the MAP decoder with PWL-LLR and $\alpha=1.7$. 


\subsection{Conclusion}

In this chapter, we have shown that the LLR-based framework can be used to justify and explain the performance of different suboptimal algorithms for Viterbi and MAP decoders. The performance of both decoders mainly depends on how the decoding algorithms approximate, implicitly or explicitly, the optimal LLR. Based on this conclusion we proposed a simple unified approach to design suboptimal decoders for coded signals in $\mathrm{S} \alpha \mathrm{S}$ noise. In the proposed approach, the suboptimal decoding algorithm should reflect a good approximation of the optimal LLR implicitly or explicitly in the calculations of the algorithm. The closer the LLR approximation is, the closer the performance of the suboptimal decoder to the optimal. For example, in case of the Viterbi decoder, we propose the dual-absolute LLR (DAbs-LLR) which has better fit compared to the Abs-LLR. The resultant DAbs-BM gives better performance than that of the Abs-BM. Moreover, when we used the PWL-LLR explicitly in the branch metric calculation, the Viterbi decoder with the PWL-LLR branch metric gives near-optimal performance with low complexity.

The same approach has been used with the MAP decoder. The resultant PWLLLR suboptimal MAP decoder shows near-optimal performance with low complexity compared to the Huber-based decoder and the optimal one. Finally, the proposed approach gives a clear way to design suboptimal decoders by using the simplest and the closest approximation to the optimal LLR. Also, there is alway a trade-off between the LLR approximation and the simplicity of the decoder. Moreover, the proposed LLR-based approach can be applied to any other decoders as long as their performance depends on the LLR such as turbo, BICM and LDPC decoders. 


\section{Chapter 7}

\section{LLR-Based Framework: Applications to Detector and Decoder Design for Signals}

\section{in Middleton's Class A noise}

\subsection{Introduction}

In this chapter, we will extend the use of the proposed LLR-based framework to design the detector and the Viterbi decoder for signals in Middelton's Class A noise. Middleton's Class A noise model $[69,70]$ is one of the statistical distributions used to model the man-made interference and the impulsive noise in different systems such as cognitive radio networks [71] and power line communication systems [72]. The optimal detector and decoder are complex due to the highly non-linear probability density function of the noise [73]. There are several approaches to design simple suboptimal receivers which vary in complexity and performance. The conventional Gaussian detector has been used as a simple suboptimal detector [73]. It gives near optimal performance with relatively higher values of the SNR, however, the performance deteriorates for moderate and low SNR values. The locally optimal Baysian 
detector has been proposed in [73] which uses the locally optimal criterion assuming weak signal reception [55]. The locally optimal detector depends on the Taylor expansion of the optimal test statistics and neglects the higher terms by using the assumption of weak signals. This detector gives better performance than the Gaussian one, however, it adds more complexity and it is still limited by the weak signal assumption which limits its performance at higher values of SNR. Other approaches use simplified forms of the probability density function (pdf) of the noise such as the use of a truncated series of the pdf as in [74]. In this chapter, we will design the PWL-LLR for the Class A noise and use it to design a simple near-optimal detector. For the decoder design, we chose to use the Viterbi decoder with the PWL-LLR as an example to extend the proposed approach for decoder design in Chapter 7 to different non-Gaussian noise models.

\subsection{Detector Design}

\subsubsection{System Model}

We will use the same system model as explained in 2.3 where a BPSK symbol $s \in$ $\{ \pm \sqrt{E}\}$ is transmitted, and $N$ independent samples per symbol are collected at the receiver. The received samples are modeled as

$$
r_{k}=s+n_{k} \quad k=1, \ldots, N
$$

where $\left\{n_{k}\right\}$ are $N$ independent Middleton's Class A noise samples. Since the Class A noise has finite power (i.e. finite noise variance), the conventional front-end receiver which uses the matched filter or the correlator is assumed to collect the received samples from the continuous received waveform at the input of the receiver. In this section, we will design a PWL-LLR with simple parameters to be used in the detector 


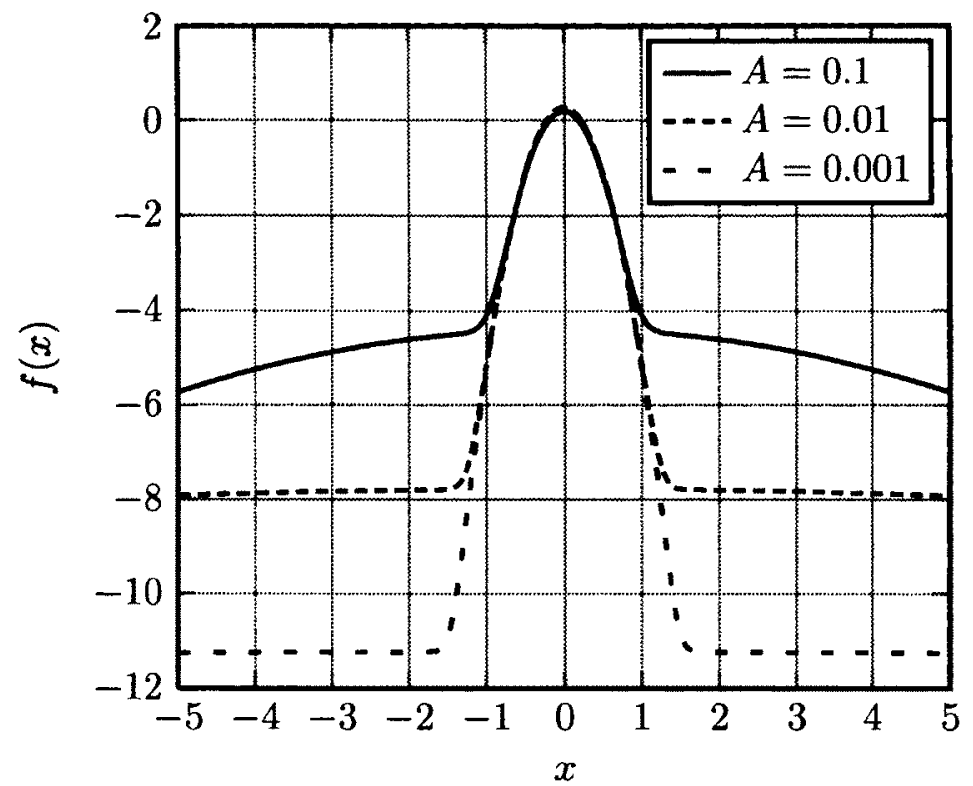

Figure 7.1: The pdf of the Class A noise on a $\log$ scale with $\Gamma=0.1$.

and the decoding design. The probability density function of Middleton's Class A noise model is given by

$$
f_{A, \Gamma}(x)=\sum_{m=0}^{\infty} \frac{e^{-A} A^{m}}{m !} \frac{1}{\sqrt{2 \pi \sigma_{m}^{2}}} \exp \left(-\frac{x^{2}}{2 \sigma_{m}^{2}}\right)
$$

where

$$
\sigma_{m}^{2}=\sigma^{2} \frac{\frac{m}{A}+\Gamma}{1+\Gamma}
$$

and $A$ is the impulsive index. When $\mathrm{A}$ is large, the impulsive noise will be continuous which leads the Class A noise to become Gaussian. $\Gamma=\sigma_{G}^{2} / \sigma_{I}^{2}$ is the Gaussian to interference noise power ratio with the Gaussian noise power $\sigma_{G}^{2}$ and the interference noise power $\sigma_{I}^{2}$ and $\sigma^{2}=\sigma_{G}^{2}+\sigma_{I}^{2}$ is the total noise power. Figures 7.1 to 7.4 show the pdf of the Class A noise with different values of $A$ and $\Gamma$ plotted on a log scale. 


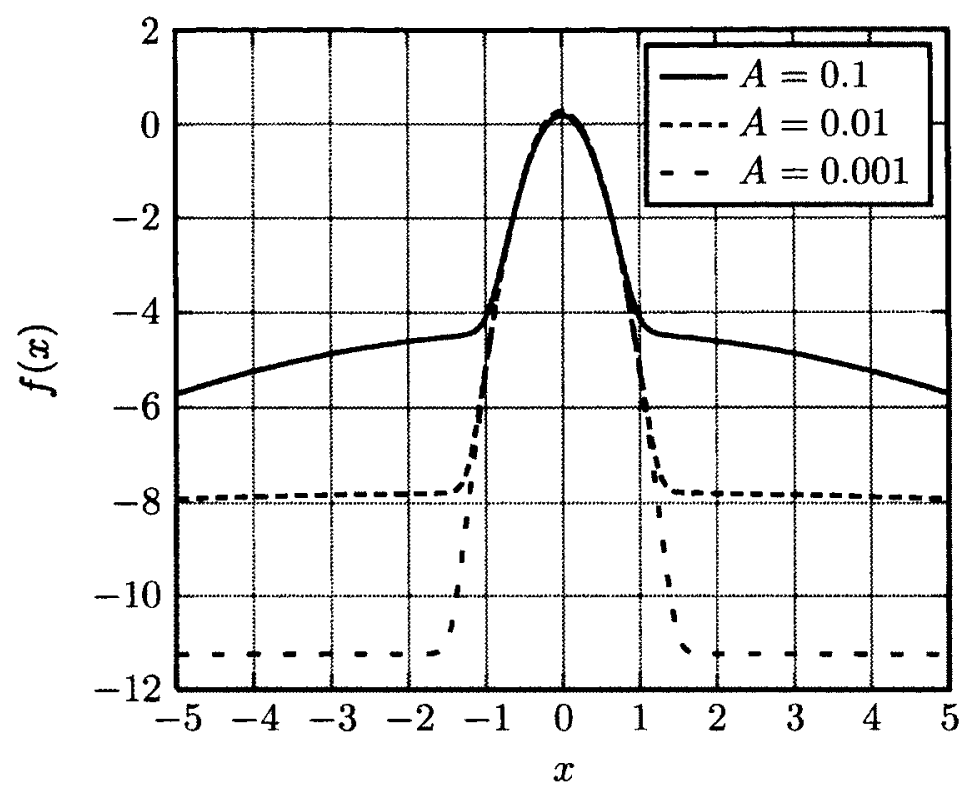

Figure 7.2: The pdf of the Class A noise on a $\log$ scale with $\Gamma=0.01$.

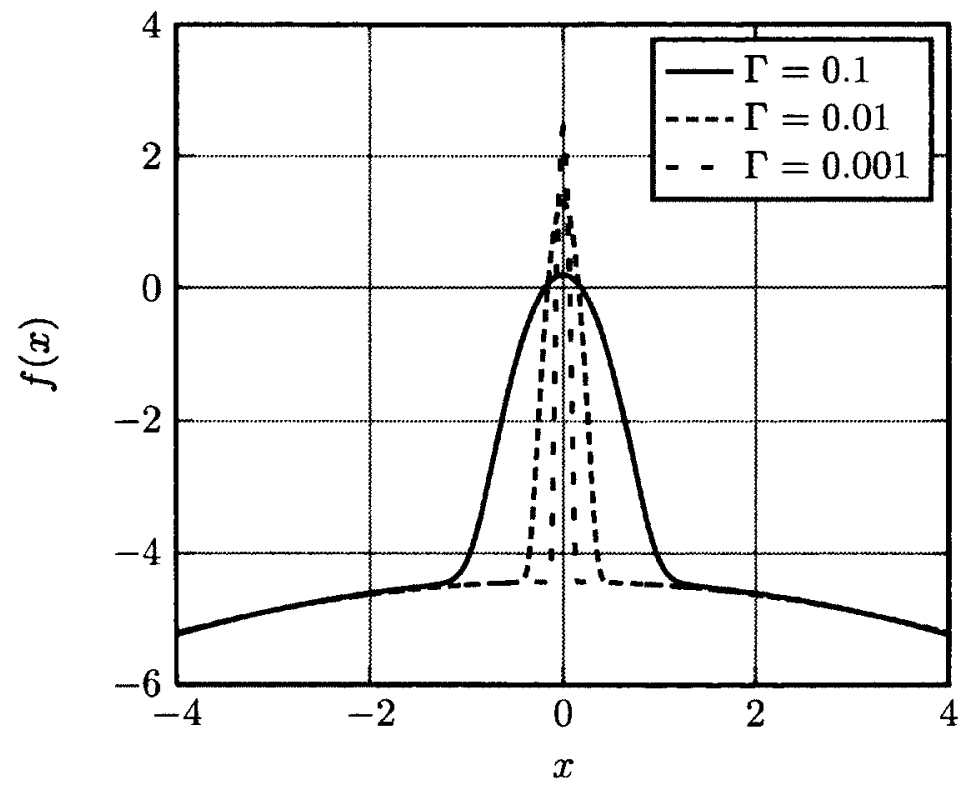

Figure 7.3: The pdf of the Class A noise on a $\log$ scale with $A=0.1$. 


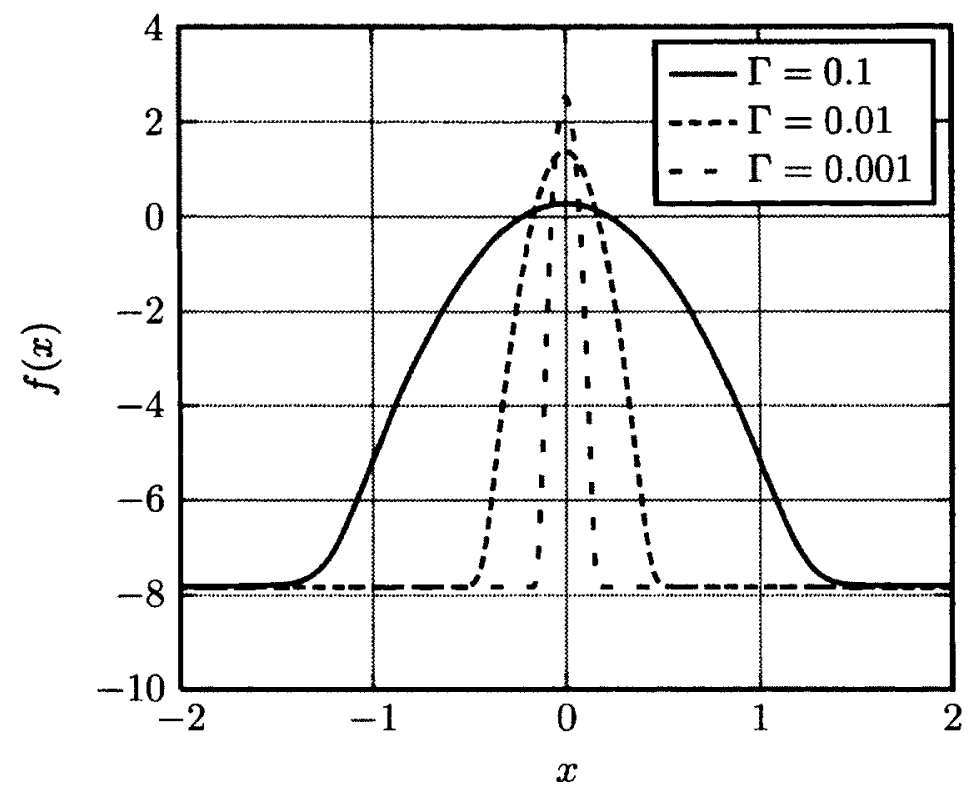

Figure 7.4: The pdf of the Class A noise on a $\log$ scale with $A=0.01$.

\subsubsection{Optimal and Suboptimal Detectors}

\section{The Optimal Maximum Likelihood Detector}

The optimal maximum likelihood (ML) detector has been analyzed in [73] by using the following test statistics for a received symbol:

$$
\lambda_{M L}=\log \left\{\frac{\prod_{k=1}^{N} f_{A, \Gamma}\left(r_{k}-\sqrt{E}\right)}{\prod_{k=1}^{N} f_{A, \Gamma}\left(r_{k}+\sqrt{E}\right)}\right\} \sum_{s_{0}}^{s_{1}} 0
$$

where $s_{0}$ is the hypothesis that $s=-\sqrt{E}$ was transmitted, and $s_{1}$ is the hypothesis that $s=+\sqrt{E}$ was transmitted. The ML detector is impractical because it requires complex computations.

\section{The Gaussian Detector}

As a simple suboptimal solution, the Gaussian detector, which uses the conventional matched filter or the correlator, has been proposed in [73] with the following test 
statistics

$$
\lambda_{\text {Gauss }}=\sum_{k=1}^{N} r_{k}
$$

It was shown that the Gaussian detector gives near optimal performance at a relatively high SNR values and lower $N$ with low and moderate impulsive noise, otherwise, it gives poor performance compared to the optimal detector.

\section{The Locally Optimal Detector}

To improve the performance, another suboptimal detector has been proposed in [73] which depends on the locally optimal criteria for weak signal reception [55]. The locally optimal detector uses the following test statistics

$$
\lambda_{L O D}=\sum_{k=1}^{N}\left(s_{1}-s_{0}\right) \frac{d}{d r_{k}} \log f_{A, \Gamma}\left(r_{k}\right) \stackrel{\substack{s_{1} \\ s_{0}}}{\gtrless} .
$$

The locally optimal detector gives better performance than the Gaussian detector at lower values of SNR, however, it adds more complexity, and it is still limited by the weak signal assumption.

In this chapter, we propose a different approach to design low complexity detectors with near optimal performance. Instead of simplifying the pdf of the noise, we propose to simplify the optimal log likelihood ratios (LLRs) in (3.2) directly. In the proposed approach, the optimal LLR is divided into three different regions where linear approximation can be used effectively which results in near optimal performance with different values of the noise parameters at low complexity approaching that of the Gaussian detector.

\subsubsection{PWL-LLR Design for Class A Noise}

In this section, we will analyze the optimal LLR graphically to understand how each term in the noise pdf in (7.2) contributes to the optimal LLR. There are different 
simplifications for the pdf of class A noise, however, they result in nonlinear design of the detector. In this section, we will propose a simplified approximation of the LLR of the Class A noise, which can be used to design simple detector with linearly approximated test statistic. Figure 7.5 shows the optimal LLR for different values of $A$ and $\Gamma$ at $0 \mathrm{~dB}$, which is given by

$$
L L R_{O p t}=\log f_{A, \Gamma}\left(r-s_{1}\right)-\log f_{A, \Gamma}\left(r-s_{0}\right)
$$

where $s_{1}$ and $s_{0}$ are the possible transmitted BPSK symbols. We can see that the LLR can be divided into three regions, a linear region, a parabolic region and a saturated flat region. We propose to use a piecewise linear approximation of the optimal LLR (PWL-LLR) as shown in Figure 7.6. To completely define the PWL-LLR, we need to calculate the boundaries $a, b$ and $c$.

Due to the anti-symmetry of the optimal LLR, we will consider only the right-hand side $(r \geq 0)$. Let us define $g_{m}(x)$ as the $m^{\text {th }}$ term in (7.2),

$$
g_{m}(x)=\frac{e^{-A} A^{m}}{m !} \frac{1}{\sqrt{2 \pi \sigma_{m}^{2}}} \exp \left(-\frac{x^{2}}{2 \sigma_{m}^{2}}\right)
$$

Figure 7.7 shows $\log f_{A, \Gamma}\left(r-s_{0}\right)$ and $\log f_{A, \Gamma}\left(r-s_{1}\right)$ along with $\log g_{m}\left(r-s_{0}\right)$ and $\log g_{m}\left(r-s_{1}\right)$ for $m=0,1,2$. From the figure, we can draw some useful conclusions to understand how each term in the conditional pdf contributes to the LLR and also to identify the boundaries of different regions and the values at each boundary.

In the linear region, where $0 \leq r \leq a$ and $b \leq r \leq c$, Figure 7.7 shows that the terms $\log g_{1}\left(r-s_{0}\right)$ and $\log g_{1}\left(r-s_{1}\right)$ can be used to approximate $\log f_{A, \Gamma}\left(r-s_{0}\right)$ and $\log f_{A, \Gamma}\left(r-s_{1}\right)$, respectively. The optimal LLR can therefore be approximated 
by

$$
\begin{aligned}
L L R_{\text {lin }} & \approx \log g_{1}\left(r-s_{1}\right)-\log g_{1}\left(r-s_{0}\right) \\
& =\frac{2 \sqrt{E} r}{\sigma_{1}^{2}} .
\end{aligned}
$$

Also, $c$ can be found by finding the intersection between $\log g_{1}\left(r-s_{1}\right)$ and $\log g_{2}\left(r-s_{1}\right)$, which is given by

$$
c=\sqrt{E}+\sqrt{\frac{2 \log \left(\frac{A \sigma_{1}}{2 \sigma_{2}}\right)}{\frac{1}{\sigma_{2}^{2}}-\frac{1}{\sigma_{1}^{2}}}}
$$

In the parabolic region $a \leq r \leq b$, the optimal LLR can be approximated by

$$
L L R_{p a r} \approx \log g_{0}\left(r-s_{1}\right)-\log g_{1}\left(r-s_{0}\right)
$$

which results in a quadratic function of $r$. The maximum of the parabolic region occurs at $r=r_{o}$ and is given by

$$
\gamma=\log \frac{\sigma_{1}}{A \sigma_{0}}+\frac{2 E}{\sigma_{1}^{2}-\sigma_{0}^{2}}
$$

where $r_{o}=\sqrt{E}(1+2 A \Gamma)$, which can be approximated by $\sqrt{E}$ for moderate and high impulsive noise where $A \Gamma \ll 1$. By equating (7.9) and (7.11), we get $a$ and $b$. To further simplify this region, we will use a piecewise linear approximation to connect $a \rightarrow \gamma \rightarrow b$ as shown in Figure 7.6.

In the flat region $(r \geq c)$, the LLR is approximated by a flat line as follows:

$$
L L R \approx \frac{2 c}{\sigma_{1}^{2}}
$$




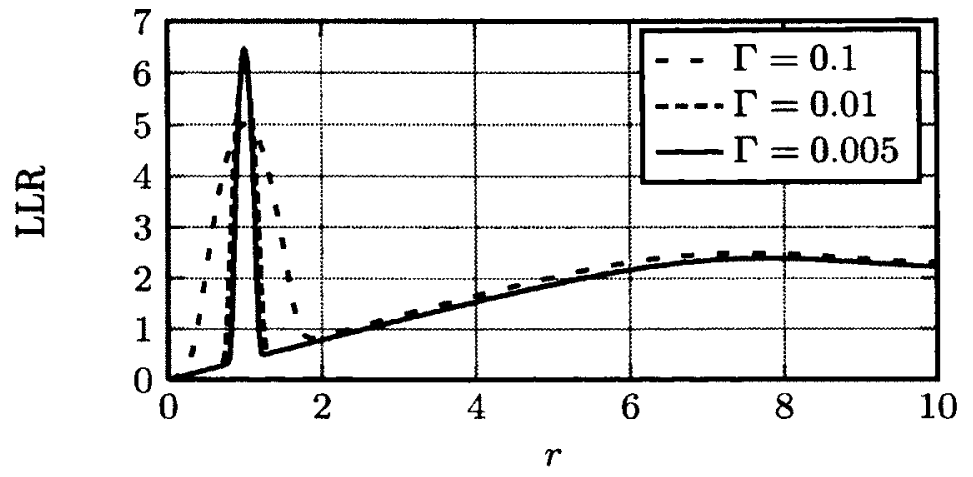

(a) LLRs with $A=0.1$ and different $\Gamma$

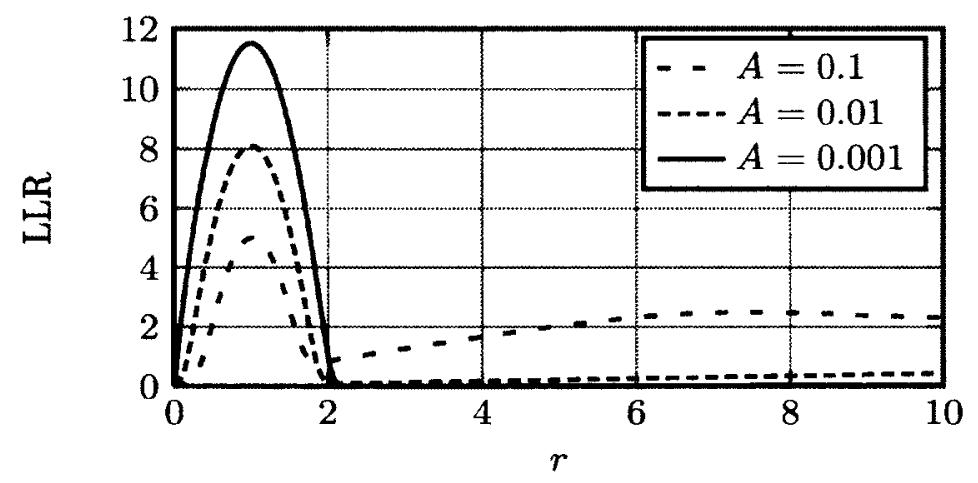

(b) LLRs with $\Gamma=0.1$ and different $A$

Figure 7.5: The optimal LLR with $\mathrm{SNR}=0 \mathrm{~dB}$.

It can be shown in Figure 7.6 that the proposed PWL-LLR has a simple implementation using linear segments which has low complexity approaching that of the Gaussian detector in (3.8).

\subsubsection{Performance Evaluation and Simulation Results}

As a performance comparison, the probability of bit error $\left(P_{b}\right)$ of the optimal and suboptimal detectors is evaluated by simulation for the case when $(A, \Gamma)$ is $(0.1,0.1)$ and $(0.1,0.01)$. The SNR is defined as $\frac{E_{s}}{N_{o}}$, where $E_{s}=N E$ is the energy per symbol and $N_{o}$ is the noise spectral density. The receiver collects $N$ independent samples per symbol. To calculate an accurate $P_{b}$, up to $10^{6}$ frames with 250 bits each are 


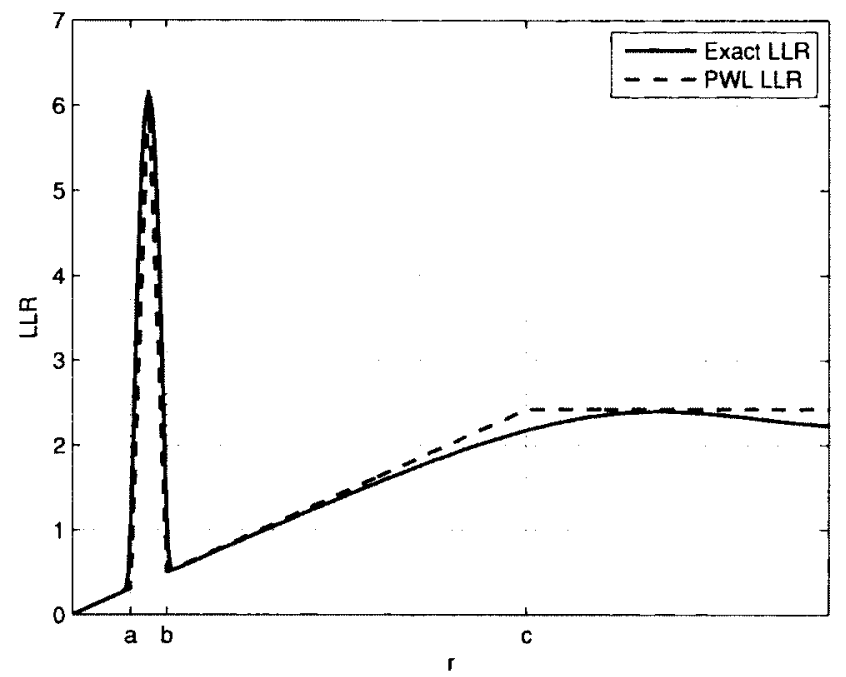

Figure 7.6: The piecewise linear (PWL) simplified LLR.

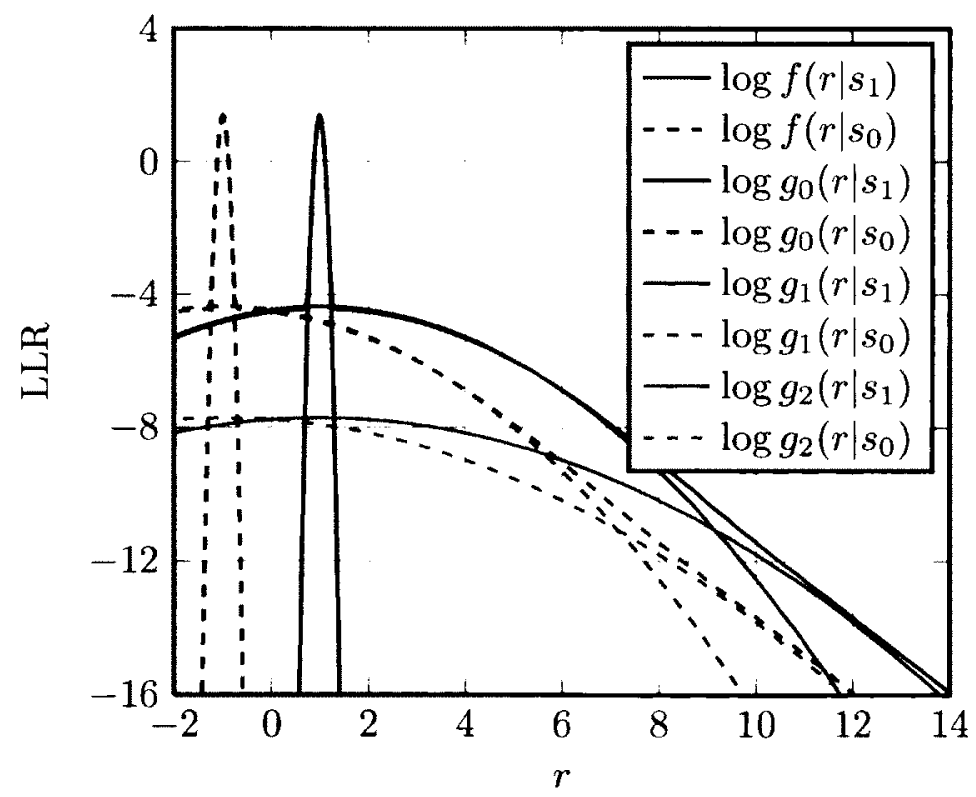

Figure 7.7: The conditional pdf, $\log f_{A, \Gamma}\left(r \mid s_{i}\right)$, and other terms. 
transmitted, and the simulation is stopped when the number of bit errors reaches $10^{3}$ or $P_{b} \leq 10^{-4}$ is achieved.

Figures 7.8 and 7.9 show the performance of the proposed PWL-LLR detector when $(A=0.1, \Gamma=0.1)$ with $N=2$ and 5 , respectively. It can be shown that the proposed detector gives near-optimal performance compared to the Gaussian one for a wide range of SNR. However, the performance of the PWL-LLR deviates from the optimal at low values of SNR, typically for SNR $<-8 \mathrm{~dB}$ when $N=2$ and SNR $<-2$ $\mathrm{dB}$ when $N=5$. The reason for that degradation is that at low values of SNR, the noise variance per received pulse becomes larger which makes the parabolic region of the LLR wider. In this case, the approximation of the parabolic region with linear segments becomes inaccurate. On the other hand, the Gaussian detector approaches the optimal one for relatively high SNR values, as can be seen in Figure 7.8 when $N=2$.

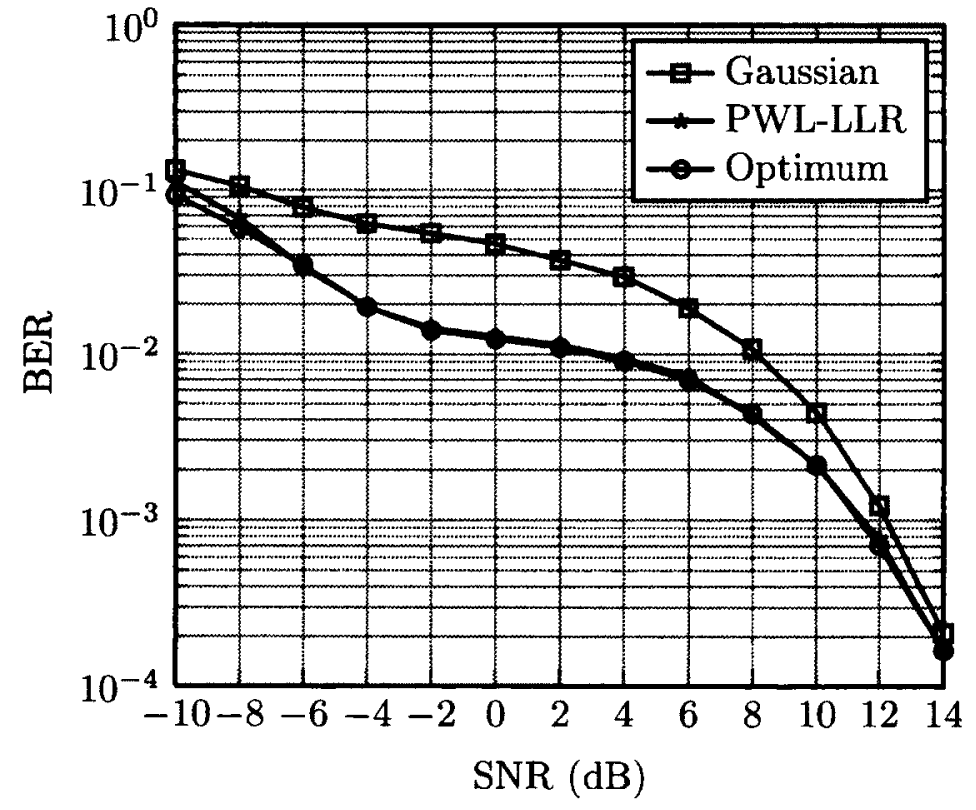

Figure 7.8: Performance comparison with $A=0.1, \Gamma=0.1$ and $N=2$. 


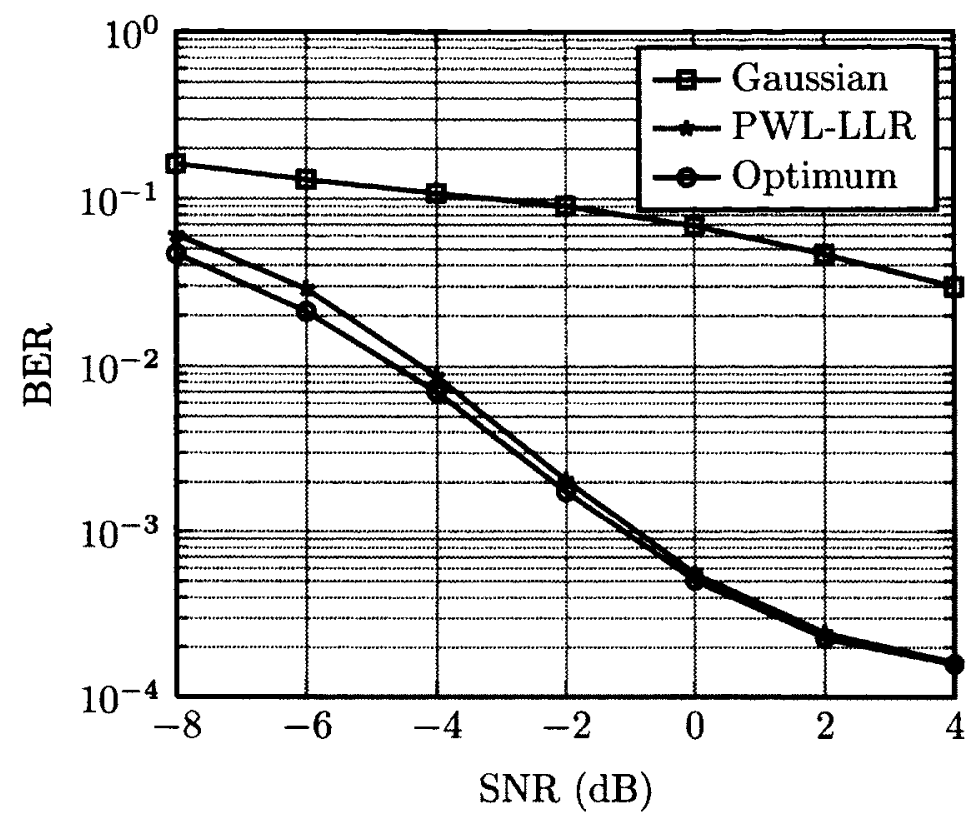

Figure 7.9: Performance comparison with $A=0.1, \Gamma=0.1$ and $N=5$.

Figures 7.10 and 7.11 show the performance of the PWL-LLR detector compared to the Gaussian one when $(A=0.1, \Gamma=0.01)$ with $N=2$ and 5 , respectively. The PWL-LLR gives near-optimal performance for relatively moderate and high SNR values with performance degradation at low SNR, typically when SNR $<-12 \mathrm{~dB}$ when $N=5$.

In summary, the PWL-LLR detector has near optimal performance due to the good fit to the optimal LLR when the SNR is large enough to give a narrow parabolic region of the LLR. On the other hand, the proposed detector has low-complexity that is almost equal to that of the Gaussian detector because both have first-order LLRs, although there are multiple regions in case of the proposed detector. 


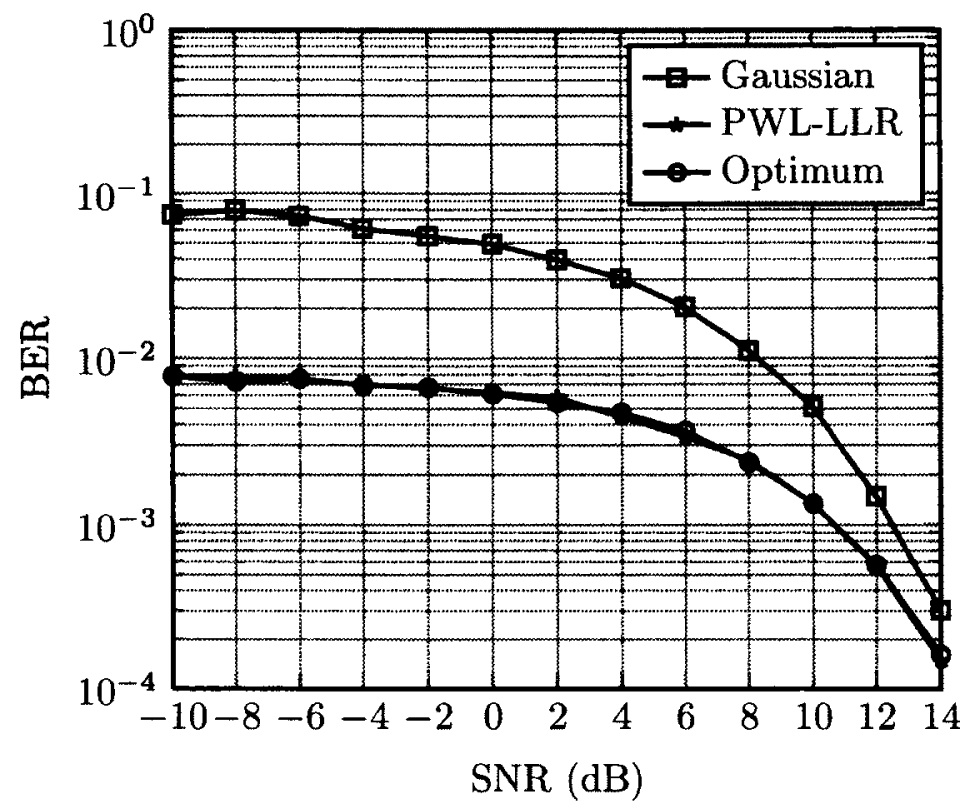

Figure 7.10: Performance comparison with $A=0.1, \Gamma=0.01$ and $N=2$.

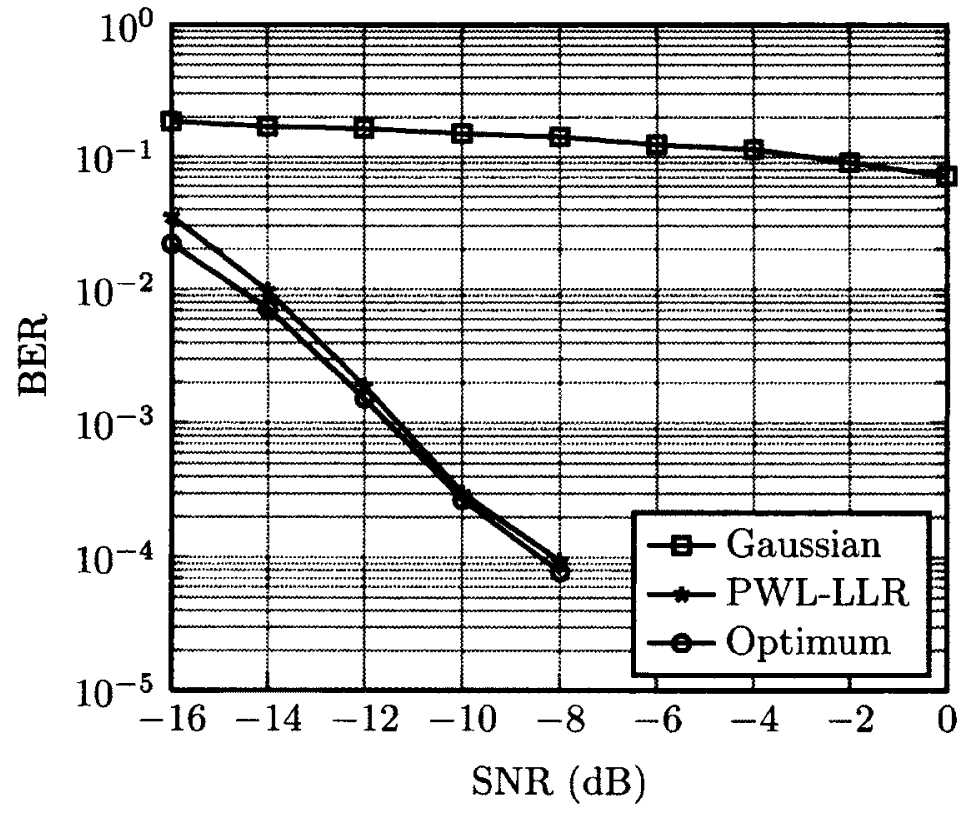

Figure 7.11: Performance comparison with $A=0.1, \Gamma=0.01$ and $N=5$. 


\subsection{Decoder Design}

In this section, the design of a simplified Viterbi decoder for signals in Middleton Class A noise is considered. The conventional Viterbi decoder with a branch metric optimized for Gaussian noise, performs poorly in the Class A noise. The optimal maximum likelihood (ML) branch metric is difficult to simplify due to the complexity of the probability density function of the noise. There are different alternatives to design low complexity Viterbi decoders which are based on simplified models of the Class A noise. A nonlinear preprocessor has been proposed to improve the performance of the Gaussian Viterbi decoder with Class A noise based on a simplified expression of the probability density function of the noise. In this chapter, we propose different approach to design the Viterbi decoder with simple linear branch metrics by using a simplified linear approximation of the log likelihood ratio. The proposed approach results in a near-optimal performance with low complexity.

\subsubsection{Optimal and Suboptimal Viterbi Decoding}

At time instant $i$, a group of $k_{c}$ message bits are applied to a rate $R_{c}=k_{c} / n_{c}$ convolutional encoder. The resulting $n_{c}$ code bits are mapped to BPSK symbols which are transmitted over an additive Middleton's Class A noise channel. The received samples are

$$
r_{i j}=c_{i j}+w_{i j}
$$

where $c_{i j} \in\left\{ \pm \sqrt{E_{c}}\right\}$ is the BPSK symbol corresponding to the $j^{\text {th }}$ code bit $(j \in$ $\left.\left\{1,2, \ldots, n_{c}\right\}\right)$ produced at time $i$, and $\left\{w_{i j}\right\}$ are independent Class A noise samples. The average transmitted energy per message bit is $E_{b}=E_{c} / R_{c}$.

The Viterbi algorithm can be used to perform the optimal maximum likelihood (ML) sequence decoding of the received samples. The ML branch metric (ML-BM) 
of the Viterbi algorithm at the $i^{\text {th }}$ time instant is:

$$
\mu_{M L}=-\sum_{j=1}^{n_{c}} \log f_{A, \Gamma}\left(r_{i, j} \mid c_{i, j}\right)
$$

where $f_{A, \Gamma}\left(r_{i, j} \mid c_{i, j}\right)$ is the conditional probability density function (pdf) of the received sample conditioned on the transmitted code bit. For simplicity, we will drop the time index, $i$, since the following analysis is applied to any time instant.

The ML branch metric requires complex computations to calculate the conditional pdf which makes the ML Viterbi algorithm impractical. The Viterbi decoder optimized for Gaussian noise is a simple alternative with branch metric (Gauss-BM):

$$
\mu_{G a u s s}=\sum_{j=1}^{n_{c}}\left(r_{j}-c_{j}\right)^{2}
$$

However, the Viterbi decoder with the Gauss-BM gives poor performance compared to the optimal. To improve the decoder performance and maintain affordable complexity, a nonlinear preprocessor was introduced in [74] to process the received signal before applying the Gaussian Viterbi decoder.

In this Chapter, we propose to use a suboptimal branch metric based on the PWL-LLR (discussed in 7.2.3)

$$
\mu_{L L R}=-\sum_{j=1 \mid c_{j}^{0}= \pm 1}^{n_{c}} \operatorname{PWL-LLR}\left(r_{j}\right)
$$

\subsubsection{Performance of the PWL-LLR Viterbi Decoder}

As a performance comparison, the probability of bit error $\left(P_{b}\right)$ of the Viterbi decoder with optimal and suboptimal branch metrics is evaluated by simulation for the case when $A=0.1$ and $\Gamma=0.01$. A $1 / 2$ rate convolutional code is used with generator polynomials $(5)_{8}$ and $(7)_{8}$. To calculate an accurate $P_{b}$, up to $10^{6}$ frames with 400 bits 
each are transmitted, and the simulation is stopped when the number of bit errors reaches $10^{3}$ or $P_{b} \leq 10^{-6}$ is achieved. The performance of the proposed suboptimal Viterbi decoder with simplified LLR-based BM is compared with that of the Viterbi decoder with the Gaussian and the optimal branch metrics. Figure 7.12 shows that the Viterbi decoder with the proposed piecewise linear simplified LLR gives near optimal performance compared to the Gaussian Viterbi decoder. The proposed simplified LLR can be used with any value of $A$ and $\Gamma$.

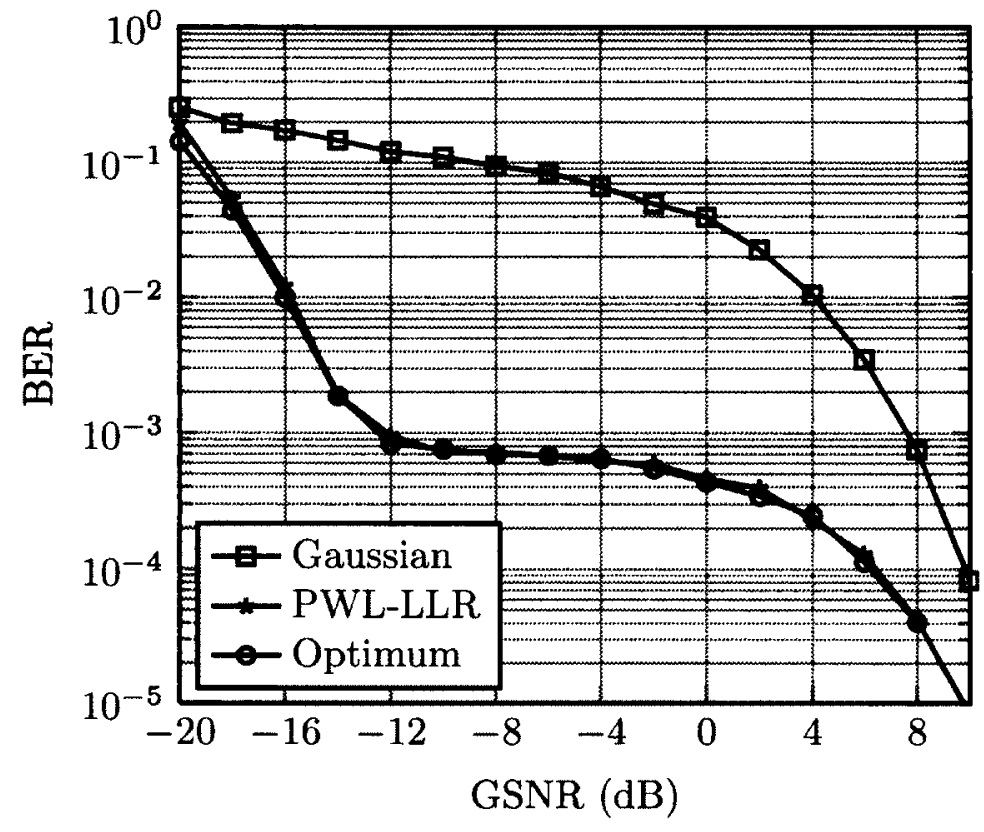

Figure 7.12: Performance of the proposed PWL LLR Viterbi decoder with $A=0.1$ and $\Gamma=0.01$.

\subsubsection{Conclusion}

In this chapter, we have demonstrated the extension of the LLR-based framework to design simple detector and decoder for signals in Class A noise. The proposed PWL-LLR has linear segments with reasonable complexity compared to the Gaussian LLR and less complexity compared to the optimal one. The PWL-LLR detector 
shows near-optimal performance with low complexity compared to the Gaussian one. Also, the PWL-LLR Viterbi decoder gives very close performance to the optimal. It has been shown that the proposed framework is applicable to design low-complexity detectors and decoders for any non-Guassian noise other than $\mathrm{S} \alpha \mathrm{S}$ noise. 


\section{Chapter 8}

\section{Conclusion and Future Work}

\subsection{Conclusion}

Motivated by the use of the symmetric alpha stable distribution to model nonGaussian impulsive noise sources in many communication systems, this thesis addresses the problem of designing low complexity receivers for signals in S $\alpha \mathrm{S}$ noise. In this thesis, we consider only the design of suboptimal detectors and decoders. The optimal detector and decoder for signals in $\mathrm{S} \alpha \mathrm{S}$ noise are too complex because of the lack of a closed form expression of the probability density function of the $\mathrm{S} \alpha \mathrm{S}$ distribution. To design low complexity detectors or decoders, different research works build on the Gaussian assumption and propose to use different preprocessing devices to process the noise impulses due the heavy tail $\mathrm{S} \alpha \mathrm{S}$ noise. The intention is to shape the noise such that it looks like Gaussian and therefore, a Gaussian detector/decoder can be applied. Most of the effort has been done to design effective and simple preprocessing devices.

In this thesis, we propose two frameworks to design near-optimal detectors with low complexity. The first framework, proposed in Chapter 4, is based on the decision regions where the behavior of the optimal detector is analyzed by using the optimal decision regions. The proposed framework gives a solid understanding of the effect of 
the noise impulses on the optimal decisions which can be used constructively, rather than clipping or removing them, to get better decisions. Based on the decisionregions framework, an adaptive soft limiter detector is proposed. This detector gives superior performance that approaches the optimal one at almost no complexity over the conventional Gaussian detector for the case of large number of samples per symbol. Moreover, a piecewise detector, which linearly approximates the optimal decision regions, has been proposed to give near-optimal performance when using only two samples per symbol at the receiver $(N=2)$ with low complexity. However, it can not be generalized for $N>2$ because of the increasing complexity of the optimal decision regions for dimensions higher than 2.

To overcome the limitations of the decision-regions framework, the second framework, which is based on the log-likelihood ratio (LLR), has been proposed in Chapter 5. In the proposed framework, the suboptimal detector uses a piecewise linear (PWL) approximation of the one-dimensional optimal LLR. When the approximated LLR has a close fit with the optimal one, the resultant decoder gives near-optimal performance with any value of $\alpha$ and $N$ at low complexity compared to the optimal detector. The PWL-LLR approximation was also used to design simple suboptimal combiner for signals in frequency-flat multipath fading channel with superior performance close to the optimal compared to many other combiners.

The proposed LLR-based framework has been also used to design low-complexity decoders for coded signals in $\mathrm{S} \alpha \mathrm{S}$ noise. We have developed a simple, yet effective approach to design simple decoders with near-optimal performance. In the proposed approach, a good approximation of the optimal LLR should be used implicitly or explicitly by the suboptimal decoding algorithm. The closer the LLR approximation is to the exact LLR, the closer the performance of the suboptimal decoder to the optimal performance. Based on this approach, a simplified branch metric has been 
proposed to improve the performance of the Viterbi decoder. However, the performance was still behind the optimal for lower values of $\alpha$ because of the inaccurate approximation of the optimal LLR used by the proposed branch metric. To get robust near-optimal performance, we proposed a new branch metric which uses the PWL-LLR explicitly. The PWL-LLR-based Viterbi decoder gives near-optimal performance at low complexity compared to many other solutions. Moreover, the same approach has been used to design PWL-LLR-based MAP decoder which also gives near optimal performance with low complexity due to the PWL-LLR approximation of the optimal LLR. The proposed methodology to design a suboptimal decoder using the PWL-LLR approximation can be used with different types of decoders such as the turbo, LDPC and BICM decoders.

Furthermore, it has been shown that the proposed framework can be easily extended to design receivers for different non-Gaussian environments other than the $\mathrm{S} \alpha \mathrm{S}$ noise such as the Middleton's Class A noise model. A simple PWL-LLR has been proposed for the case of Class A noise to design a simple suboptimal detector with near-optimal performance. Also, it has been used with the decoding approach to design a simple Viterbi decoder with performance close to the optimal.

In summary, this thesis gives new approaches to design low complexity suboptimal detectors and decoders for signals in $\mathrm{S} \alpha \mathrm{S}$ noise which can be extended to other nonGaussian noise models. Instead of improving the receiver optimized for the Gaussian noise, we use the optimal decision regions and the optimal LLR as different ways to understand the behavior of the optimal detector and decoder. As a result, a simple approximations of the LLR has been used to design suboptimal detectors and decoders. The proposed design approach can be applied to any non-Gaussian noise with distributions that result in complex receivers. 


\subsection{Future Work}

\subsubsection{Channel Estimation in $\mathrm{S} \alpha \mathrm{S}$}

Motivated by the findings of this thesis there are several outstanding problems that need to be addressed to design different parts of the receiver in $\mathrm{S} \alpha \mathrm{S}$ noise. One such problem invokes channel estimation, which has been extensively studied, but mostly only for the case of Gaussian noise. Almost all the channel estimation algorithms depend on two main estimation techniques in the context of the statistical signal processing; the maximum likelihood (ML) parameter estimation and the minimum mean square error (MMSE) parameter estimation. Each technique has its own advantages and disadvantages in terms of the estimation accuracy and complexity. When the noise has a $\mathrm{S} \alpha \mathrm{S}$ distribution, there are some limitations on the use of those estimation techniques [8]. The ML estimation techniques become more complex because there is no closed form expression for the probability density function of the noise. On the other hand, the MMSE estimation techniques are no longer valid because they depend on minimizing the estimation error variance whereas the second order moment of $\mathrm{S} \alpha \mathrm{S}$ random variables is infinite.

To overcome the complexity of the ML estimation technique, it should be possible to find a unified approach similar to the optimal decision regions and the optimal LLR used in the detection and decoding part, respectively, such that it can have a simple approximation. Also, to overcome the limitation of using the MMSE estimation technique, the use of fractional lower order statistics which depends on the fact that the $\mathrm{S} \alpha \mathrm{S}$ random variable possess a finite absolute moment for orders less than $\alpha$ is proposed [8] [17],i.e.,

$$
\left.E \|\left. X\right|^{p}\right]<\infty \quad \text { if } \quad p<\alpha
$$

Based on the fractional lower order statistics, we propose to develop different blind 
and pilot-based channel estimation algorithms.

Moreover, since the $\mathrm{S} \alpha \mathrm{S}$ distribution has a closed form expression for its characteristic function, we propose to investigate the possibility of designing estimation algorithms by using the characteristic function instead of using the probability density function.

\subsubsection{LLR Estimation}

Motivated by the use of the PWL-LLR to design simple detectors and decoders for signals in $\mathrm{S} \alpha \mathrm{S}$ noise, we propose to directly estimate the LLR at the receiver, based initially on a training sequence to update with decision feedback. For different environments where the noise is non-Gaussian, especially when there is interference with unknown statistics, it will be helpful if the receiver has the ability to estimate the optimal LLR or at least estimate a linear fit of the optimal LLR. 


\section{List of References}

[1] K. L. Blackard, T. S. Rappaport, and C. W. Bostian, "Measurements and models of radio frequency impulsive noise for indoor wireless communications," IEEE Journal on Selected Areas in Communications, vol. 11, no. 7, pp. 991-1001, Sept. 1993.

[2] T. Blankenship, D. Kriztman, and T. Rappaport, "Measurements and simulation of radio frequency impulsive noise in hospitals and clinics," in IEEE 47th Vehicular Technology Conference, vol. 3, May 1997, pp. 1942-1946.

[3] D. Middleton, "Man-made noise in urban environments and transportation systems: Models and measurements," IEEE Transactions on Communications, vol. 21, no. 11, pp. 1232-1241, Nov. 1973.

[4] _ - "Statistical-physical models of electromagnetic interference," IEEE Transactions on Electromagnetic Compatibility, vol. 19, no. 3, pp. 106-127, Aug. 1977.

[5] G. Madi, B. Vrigneau, Y. Pousset, R. Vauzelle, and B. L. Agba, "Impulsive noise of partial discharge and its impact on minimum distance-based precoder of MIMO system," in 18th European Signal Processing Conference, Aalborg, Denmark, August 2010.

[6] W. Wan Abdullah, T. Chuah, A. Zainal Abidin, and M. Jenu, "Measurement and verification of the impact of electromagnetic interference from household appliances on digital subscriber loop systems," IET Science, Measurement Technology, vol. 3, no. 6, pp. 384-394, 2009.

[7] D. Parson, The Mobile Radio Propagation Channel. New York: Wiley, 1996.

[8] C. L. Nikias and M. Shao, Signal Processing with Alpha-Stable Distributions and Applications. John Wiley \& Sons Canada, 1995. 
[9] M. Win, P. Pinto, and L. Shepp, "A mathematical theory of network interference and its applications," Proceedings of the IEEE, vol. 97, no. 2, pp. 205-230, Feb. 2009 .

[10] M. Nassar, K. Gulati, M. R. DeYoung, B. L. Evans, and K. R. Tinsley, "Mitigating near-field interference in laptop embedded wireless transceivers," Journal of Signal Processing Systems, March 2009.

[11] S. Niranjayan and N. C. Beaulieu, "A myriad filter detector for UWB multiuser communication," in IEEE International Conference on Communications, Beijing, May 2008, pp. 3918-3922.

[12] D. Middleton, "Channel modeling and threshold signal processing in underwater acoustics: An analytical overview," IEEE Journal of Oceanic Engineering, vol. 12, no. 1, pp. 4-28, Jan. 1987.

[13] P. L. Brockett, M. Hinich, and G. R. Wilson, "Nonlinear and non-Gaussian ocean noise," The Journal of the Acoustical Society of America, vol. 82, no. 4, pp. 1386-1394, 1987. [Online]. Available: http://link.aip.org/link/?JAS/82/1386/1

[14] E. J. Wegman, S. C. Schwartz, and J. B. Thomas, Topics in Non-Gaussian Signal Processing. New York: Springer, 1989.

[15] M. Zimmermann and K. Dostert, "Analysis and modeling of impulsive noise in broad-band powerline communications," IEEE Transactions on Electromagnetic Compatibility, vol. 44, no. 1, pp. 249-258, Feb. 2002.

[16] R. Kapoor, A. Banerjee, G. A. Tsihrintzis, and N. Nandhakumar, "UWB radar detection of targets in foliage using alpha-stable clutter models," IEEE Transactions on Aerospace and Electronic Systems, vol. 35, no. 3, pp. 819-834, Jul. 1999.

[17] G. A. Tsihrintzis and C. L. Nikias, "Evaluation of fractional, lower-order statistics-based detection algorithms on real radar sea-clutter data," IEE Proceedings on Radar, Sonar and Navigation, vol. 144, no. 1, pp. 29-38, Feb 1997.

[18] D. Middleton, "Non-Gaussian noise models in signal processing for telecommunications: New methods and results for class A and class B noise models," IEEE Transactions on Information Theory, vol. 45, no. 4, pp. 1129-1149, May 1999.

[19] D. Stein, "Detection of random signals in Gaussian mixture noise," IEEE Transactions on Information Theory, vol. 41, no. 6, pp. 1788-1801, Nov. 1995. 
[20] R. Kozick and B. Sadler, "Maximum-likelihood array processing in non-Gaussian noise with Gaussian mixtures," IEEE Transactions on Signal Processing, vol. 48, no. 12 , pp. $3520-3535$, Dec. 2000.

[21] D. Andrews and C. Mallows, "Scale mixtures of normal distributions," Journal of the Royal Statistical Society, vol. B-36, pp. 99-102, 1974.

[22] E. Kuruoglu, W. Fitzgerald, and P. Rayner, "Near optimal detection of signals in impulsive noise modeled with a symmetric $\alpha$-stable distribution," IEEE Communications Letters, vol. 2, no. 10, pp. 282-284, Oct. 1998.

[23] B. Aazhang and H. Poor, "Performance of DS/SSMA communications in impulsive channels-Part I: Linear correlation receivers," IEEE Transactions on Communications, vol. 35, no. 11, pp. 1179-1188, Nov. 1987.

[24] _ - "Performance of DS/SSMA communications in impulsive channels. Part II: Hard-limiting correlation receivers," IEEE Transactions on Communications, vol. 36, no. 1, pp. 88-97, Jan. 1988.

[25] M. Ghosh, "Analysis of the effect of impulse noise on multicarrier and single carrier QAM systems," IEEE Transactions on Communications, vol. 44, no. 2, pp. 145-147, Feb. 1996.

[26] X. Wang and H. Poor, "Robust multiuser detection in non-Gaussian channels," IEEE Transactions on Signal Processing, vol. 47, no. 2, pp. 289-305, Feb. 1999.

[27] D. Middleton, "Canonical and quasi-canonical probability models of class A interference," IEEE Transactions on Electromagnetic Compatibility, vol. 25, no. 2, pp. 76-106, May 1983.

[28] K. Gulati, A. Chopra, B. Evans, and K. Tinsley, "Statistical modeling of cochannel interference," in IEEE Global Telecommunications Conference, Honolulu, USA, Nov. 2009.

[29] E. Sousa, "Interference modeling in a direct-sequence spread-spectrum packet radio network," IEEE Transactions on Communications, vol. 38, no. 9, pp. 14751482, Sep. 1990.

[30] J. Ilow and D. Hatzinakos, "Analytic alpha-stable noise modeling in a Poisson field of interferers or scatterers," IEEE Transactions on Signal Processing, vol. 46, no. 6, pp. 1601-1611, June 1998. 
[31] J. Ilow, "Signal processing in alpha-stable noise environments: Noise modeling, detection and estimation." Ph.D. dissertation, University of Toronto, 1996.

[32] H. El Ghannudi, L. Clavier, N. Azzaoui, F. Septier, and R.-A. Rolland, " $\alpha$-stable interference modeling and Cauchy receiver for an IR-UWB ad hoc network," IEEE Transactions on Communications, vol. 58, no. 6, pp. 1748-1757, June 2010 .

[33] J. Chen, L. Clavier, Y. Xi, A. Burr, N. Rolland, and P. Rolland, "Alpha-stable interference modelling and relay selection for regenerative cooperative IR-UWB systems," in European Wireless Technology Conference (EuWiT), Paris, Sep. 2010 .

[34] N. Azzaoui and L. Clavier, "Statistical channel model based on $\alpha$-stable random processes and application to the $60 \mathrm{GHz}$ ultra wide band channel," IEEE Transactions on Communications, vol. 58, no. 5, pp. 1457-1467, May 2010.

[35] A. Achim, E. Kuruoglu, and J. Zerubia, "SAR image filtering based on the heavy-tailed Rayleigh model," IEEE Transactions on Image Processing, vol. 15, no. 9, pp. 2686-2693, Sept. 2006.

[36] A. Briassouli and M. Strintzis, "Locally optimum nonlinearities for DCT watermark detection," IEEE Transactions on Image Processing, vol. 13, no. 12, pp. 1604-1617, Dec. 2004.

[37] A. Briassouli, P. Tsakalides, and A. Stouraitis, "Hidden messages in heavy-tails: DCT-domain watermark detection using alpha-stable models," IEEE Transactions on Multimedia, vol. 7, no. 4, pp. 700-715, Aug. 2005.

[38] T. Wan, N. Canagarajah, and A. Achim, "Segmentation-driven image fusion based on alpha-stable modeling of wavelet coefficients," IEEE Transactions on Multimedia, vol. 11, no. 4, pp. 624-633, June 2009.

[39] H. Bhaskar, L. Mihyalova, and A. Achim, "Automatic object detection based on adaptive background subtraction using symmetric alpha stable distribution," in IET Seminar on Target Tracking and Data Fusion: Algorithms and Applications, April 2008, pp. 197-203.

[40] S. Liao and A. Chung, "Feature based nonrigid brain MR image registration with symmetric alpha stable filters," IEEE Transactions on Medical Imaging, vol. 29, no. 1, pp. 106-119, Jan. 2010. 
[41] A. Achim, A. Bezerianos, and P. Tsakalides, "Wavelet-based ultrasound image denoising using an alpha-stable prior probability model," in International Conference on Image Processing, vol. 2, Oct. 2001, pp. 221-224.

[42] D. Zha, "Novel ultrasound images shot noise removal algorithm based on 2-D wavelet decomposition and stable distribution model," in The 1st International Conference on Bioinformatics and Biomedical Engineering, July 2007, pp. 845848.

[43] T. Qiu, D. Zha, W. Guo, D. Guo, and Z. Chen, "Blind estimation of evoked potentials in alpha stable distribution environments," in 27th Annual International Conference of the Engineering in Medicine and Biology Society, Jan. 2005, pp. 2021-2024.

[44] V. M. Zolotarev, One-dimensional Stable Distributions. American Mathematical Society, 1986.

[45] M. S. T. Gennady Samorodnitsky, Stable non-Gaussian Random Processes: Stochastic models with infinite variance. Boca Raton: Chapman and Hall/CRC, 1994.

[46] A. W. Aleksander Janicki, Simulation and Chaoic Behavior of $\alpha$-stable stochastic processes. New York: Marcel Dekker, Inc., 1994.

[47] W. Feller, An Introduction to Probability Theory and its Applications. New York: John Wiley and Sons Inc., 1966.

[48] S. Ambike, J. Ilow, and D. Hatzinakos, "Detection for binary transmission in a mixture of Gaussian noise and impulsive noise modeled as an alpha-stable process," IEEE Signal Processing Letters, vol. 1, no. 3, pp. 55-57, Mar 1994.

[49] J. G. Gonzalez, "Robust techniques for wireless communications in non-Gaussian environments," Ph.D. dissertation, University of Delaware, Newark, DE, USA, 1997.

[50] G. Tsihrintzis and C. Nikias, "Performance of optimum and suboptimum receivers in the presence of impulsive noise modeled as an alpha-stable process," IEEE Transactions on Communications, vol. 43, no. 234, pp. 904-914, Feb/Mar/Apr 1995.

[51] A. Swami and B. M. Sadler, "On some detection and estimation problems in heavy-tailed noise," Signal Processing, vol. 82, no. 12, pp. 1829-1846, 2002. 
[52] S. Winograd, Arithmetic complexity of computations. Society for Industrial and Applied Mathematics (SIAM), 1980.

[53] A. Li, Y. Wang, W. Xu, and Z. Zhou, "Receiver design of MIMO systems in a mixture of Gaussian noise and impulsive noise," in IEEE 60th Vehicular Technology Conference, vol. 2, Sept. 2004, pp. 1493-1497.

[54] J. G. Gonzalez and G. R. Arce, "Statistically-efficient filtering in impulsive environments: Weighted myriad filters," EURASIP Journal on Applied Signal Processing, vol. 2002, no. 1, pp. 4-20, 2002.

[55] S. A. Kassam, Signal Detection in Non-Gaussian Noise. New York: SpringerVerlag, 1988.

[56] B. Sadler, "Detection in correlated impulsive noise using fourth-order cumulants," IEEE Transactions on Signal Processing, vol. 44, no. 11, pp. 2793-2800, Nov 1996.

[57] S. Niranjayan and N. Beaulieu, "The BER optimal linear rake receiver for signal detection in symmetric alpha-stable noise," IEEE Transactions on Communications, vol. 57, no. 12, pp. 3585-3588, Dec. 2009.

[58] A. Rajan and C. Tepedelenlioglu, "Diversity combining over Rayleigh fading channels with symmetric alpha-stable noise," IEEE Transactions on Wireless Communications, vol. 9, no. 9, pp. 2968-2976, Sept. 2010.

[59] M. Chitre, J. Potter, and S. Ong, "Viterbi decoding of convolutional codes in symmetric $\alpha$-stable noise," IEEE Transactions on Communications, vol. 55, no. 12 , pp. 2230-2233, Dec. 2007.

[60] T. Summers and S. Wilson, "Turbo code performance in heavy tailed noise," in Proc. CISS'98, Princeton, NJ, USA, 1998.

[61] T. Oberg and M. Mettiji, "Robust detection in digital cofmmunications," IEEE Transactions on Communications, vol. 43, no. 5, pp. 1872-1876, May 1995.

[62] X. Hu, C. Zhao, and X. H. Yu, "A robust Viterbi decoder and its application to terrestrial HDTV broadcasting," IEEE Transactions on Broadcasting, vol. 43, no. 2, pp. 227-234, Jun. 1997.

[63] L. Bahl, J. Cocke, F. Jelinek, and J. Raviv, "Optimal decoding of linear codes for minimizing symbol error rate (corresp.)," IEEE Transactions on Information Theory, vol. 20, no. 2, pp. 284-287, March 1974. 
[64] P. Robertson, E. Villebrun, and P. Hoeher, "A comparison of optimal and suboptimal MAP decoding algorithms operating in the log domain," in IEEE International Conference on Communications, Seattle, USA, vol. 2, Jun. 1995, pp. 1009-1013.

[65] J. Woodard and L. Hanzo, "Comparative study of turbo decoding techniques: an overview," IEEE Transactions on Vehicular Technology, vol. 49, no. 6, pp. 2208-2233, Nov. 2000.

[66] P. Huber, Robust Statistics. Wiley, 1981.

[67] T. Chuah, "Distance metric for soft-decision decoding in non-Gaussian channels," Electronics Letters, vol. 39, no. 14, pp. 1062-1063, July 2003.

[68] T. C. Chuah, "Robust iterative decoding of turbo codes in heavy-tailed noise," IEE Proceedings Communications, vol. 152, no. 1, pp. 29-38, Feb. 2005.

[69] D. Middleton, "Statistical-physical models of electromagnetic interference," IEEE Transactions on Electromagnetic Compatibility, vol. EMC-19, no. 3, pp. 106-127, aug. 1977.

[70] - "Non-Gaussian noise models in signal processing for telecommunications: New methods and results fro class A and class B noise models," IEEE Transactions on Information Theory, vol. 45, no. 4, pp. 1129-1149, March 1999.

[71] K. Gulati, B. Evans, J. Andrews, and K. Tinsley, "Statistics of co-channel interference in a field of poisson and poisson-poisson clustered interferers," IEEE Transactions on Signal Processing, vol. 58, no. 12, pp. 6207-6222, dec. 2010.

[72] A. Sergienko, M. Sidorov, and E. Kozhevnikov, "Performance of coded DMT system with account for temporal dynamics of impulsive noise," in IEEE Region 8 International Conference on Computational Technologies in Electrical and Electronics Engineering, 2008, july 2008, pp. 430-435.

[73] A. D. Spaulding and D. Middleton, "Optimum reception in an impulsive interference environment - Part I: Coherent detection," IEEE Transactions on Communication, vol. COM-25, no. 9, pp. 910-923, Sep. 1977.

[74] D. U. M. K. Yuki Nakano and Y. Morihiro, "Viterbi decoding for convolutional code over class a noise channel." in International Symposium on Power-Line Communications and Its Applications, Kyoto, Japan $7^{\text {th }}, 2003$. 